KAPL-4823

UC-32

(DOE/TIC-4500-R75)

\title{
Monte Carlo Fundamentals
}

F. B. BROWN and T. M. SUTTON

February 1996

\author{
Prepared by \\ Lockheed Martin Company \\ KNOLLS ATOMIC POWER LABORATORY \\ Schenectady, New York
}

Contract No. DE-AC12-76-SN-00052 


\section{Disclaimer}

This report was prepared as an account of work sponsored by an agency of the United States Government. Neither the United States Government nor any agency thereof, nor any of their employees, makes any warranty, expressed or implied, or assumes any legal liability or responsibility for the accuracy, completeness, or usefulness of any information, apparatus, product, or process disclosed, or represents that its use would not infringe privately owned rights. Reference herein to any specific commercial product, process or service by trade name, trademark, manufacturer, or otherwise, does not necessarily constitute or imply its endorsement, recommendation, or favoring by the United States Government or any agency thereof. The views or opinions of authors expressed herein do not necessarily state or reflect those of the United States Government or any agency thereof. 


\section{DISCLAIMER}

Portions of this document may be illegible in electronic image products. Images are produced from the best available original document. 



\section{CONTENTS}

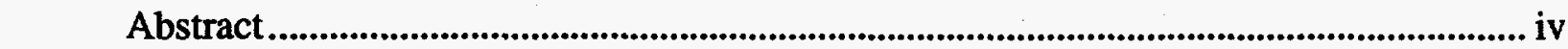

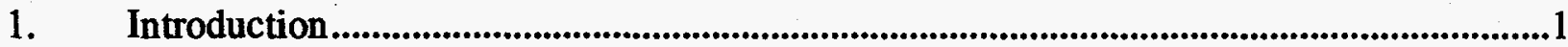

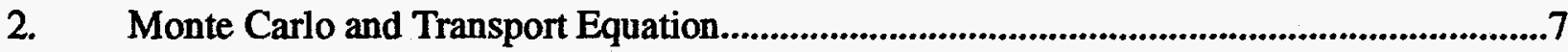

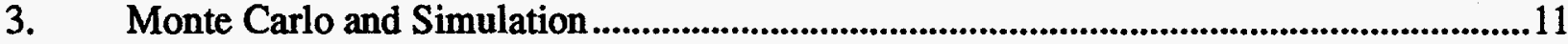

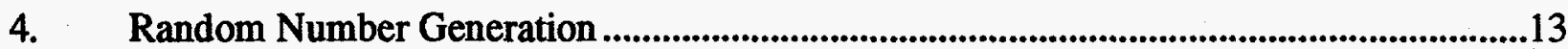

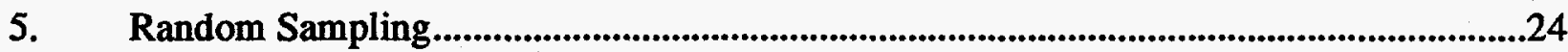

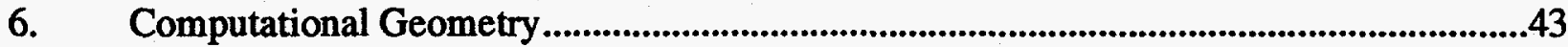

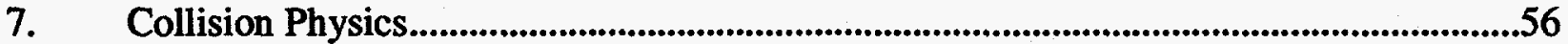

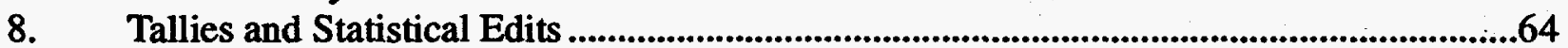

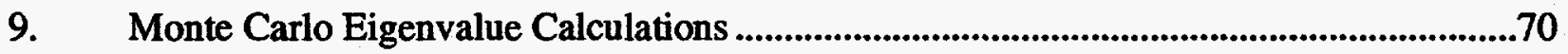

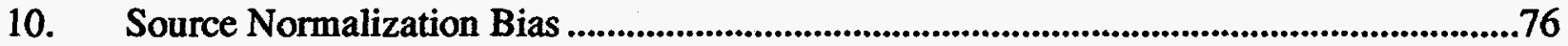

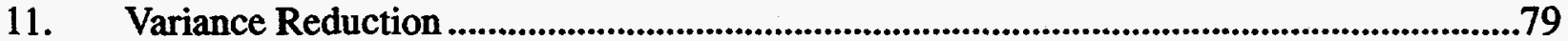

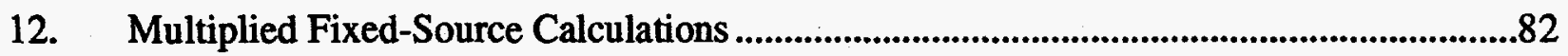

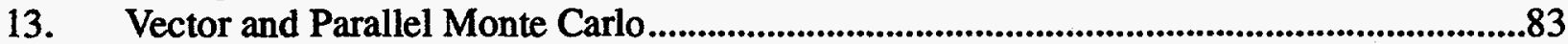

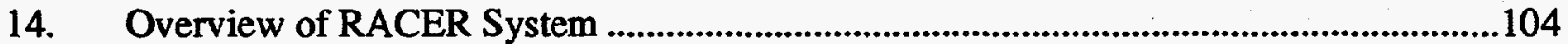

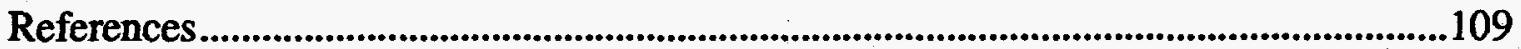




\begin{abstract}
This report is composed of the lecture notes from the first half of a 32-hour graduate-level course on Monte Carlo methods offered at KAPL. These notes, prepared by two of the principle developers of KAPL's RACER Monte Carlo code, cover the fundamental theory, concepts, and practices for Monte Carlo analysis methods. In particular, a thorough grounding in the basic fundamentals of Monte Carlo methods is presented, including random number generation, random sampling, the Monte Carlo approach to solving transport problems, computational geometry, collision physics, tallies, and eigenvalue calculations. Furthermore, modern computational algorithms for vector and parallel approaches to Monte Carlo calculations are covered in detail, including fundamental parallel and vector concepts, the event-based algorithm, master/slave schemes, parallel scaling laws, and portability issues.
\end{abstract}




\section{Introduction}

The Monte Carlo method has been used for over 40 years to solve radiation transport problems in high energy physics, nuclear reactor analysis, radiation shielding, medical imaging, oil well-logging, etc. Individual particle histories are simulated using random numbers, highly accurate representations of particle interaction probabilities, and exact models of 3D problem geometry. Monte Carlo methods are sometimes the only viable methods for analyzing complex, demanding particle transport problems.

The principal limitation on the Monte Carlo method is computer power. To achieve results with acceptably low statistical uncertainty, it is often necessary to simulate millions of particle histories, consuming many hours or days of supercomputer time. Monte Carlo methods have been successfully adapted to nearly all advanced computer architectures, including vector and parallel computers. Vector Monte Carlo codes were first developed in the early 1980's using an "eventbased" algorithm. More recently, parallel Monte Carlo codes have been successful using a "master/slave" approach.

This report is composed of the lecture notes from the first half of a 32-hour graduate-level course on Monte Carlo methods offered at KAPL. The purpose of the course is to provide thorough user education in Monte Carlo methods so that engineers can make more effective use of KAPL supercomputers. These notes, prepared by two of the principle developers of KAPL's RACER Monte Carlo code, cover the fundamental theory, concepts, and practices for Monte Carlo analysis methods. In particular, a thorough grounding in the basic fundamentals of Monte Carlo methods is presented, including random number generation, random sampling, the Monte Carlo approach to solving transport problems, computational geometry, collision physics, tallies, and eigenvalue calculations. While this material is available in standard references, a concise and coherent overview is provided. Furthermore, modern computational algorithms for vector and parallel approaches to Monte Carlo calculations are covered in detail, including fundamental parallel and vector concepts, the event-based algorithm, master/slave schemes, parallel scaling laws, and portability issues.

In addition to the lecture notes, a substantial number of references are provided. Included are general references on Monte Carlo methods for particle transport problems, random number generation and random sampling, and Monte Carlo methods for the solution of reactor eigenvalue problems; as well as open-literature publications on the RACER Monte Carlo code, vector and parallel Monte Carlo, and related Monte Carlo methods 


\section{Monte Carlo}

\section{Fundamentals}

$=$
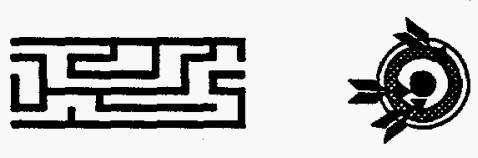

Forrest B. Brown

$\&$

Thomas M. Sutton

\section{Topics}

- Introduction

- Monte Carlo \& Transport Equation

- Monte Carlo \& Simulation

- Random Number Generation

- Random Sampling

- Computational Geometry

- Collision Physics

- Tallies \& Statistical Edits

- Monte Carlo Eigenvalue Calculations

- Source Normalization Bias

- Variance Reduction

- Multiplied Fixed-Source Calculations

- Vector \& Parallel Monte Carlo

- Overview of RACER System 
- John Von Neumann invented scientific computing in the 1940's

- stored programs, "software"

- algorithms \& flowcharts

- assisted with hardware design as well

- "ordinary" computers are called "Von Neumann machines"

- John Von Neumann invented Monte Carlo particle transport in the 1940's

- Highly accurate - no essential approximations

- Expensive - typically "method of last resort"

- Monte Carlo codes for particle transport have been proven to work effectively on all computers

SIMD, MIMD, vector, parallel, supercomputers, workstations, clusters of workstations, .....

- Two basic ways to approach the use of Monte Carlo methods for solving the transport equation:

- mathematical technique for numerical integration

- computer simulation of a physical process

$\rightarrow$ Each is "correct"

- mathematical approach useful for: importance sampling, convergence, variance reduction, random sampling techniques, .....

- simulation approach useful for: collision physics, tracking, tallying, .....

- For Monte Carlo approach, consider the integral form of the Boltzmann equation.

- Most theory on Monte Carlo deals with fixed-source problems. Eigenvalue problems are needed for reactor physics calculations 


\section{Simple Monte Carlo Example}

Evaluate $G=\int_{0}^{1} g(x) d x$, with $g(x)=\sqrt{1-x^{2}}$

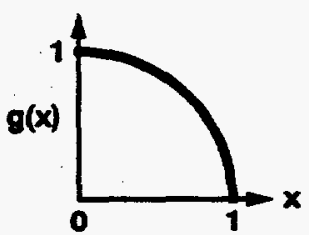

- Mathematical approach:

For $k=1, \ldots, N$ : choose $\hat{x}_{k}$ randomly in $(0,1)$

$G=(1-0) \cdot[$ average value of $g(x)] \sim \frac{1}{N} \sum_{k=1}^{N} g\left(\hat{x}_{k}\right)=\frac{1}{N} \sum_{k=1}^{N} \sqrt{1-\hat{x}_{k}^{2}}$

- Simulation approach:

"darts game"

For $k=1, \ldots, N$ : choose $\hat{x}_{k}$ and $\hat{y}_{k}$ randomly in $(0,1)$

$$
\begin{aligned}
& \qquad \text { If } \hat{\mathrm{x}}_{k}^{2}+\hat{y}_{k}^{2} \leq 1 \text {, tally a "hit" } \\
& \mathbf{G}=\text { [area under curve] } \sim(1 \cdot 1) \cdot \frac{\text { number of hits }}{N}
\end{aligned}
$$

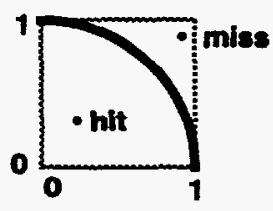

Monte Carlo is often the method-of-choice for applications with integration over many dimensions

examples: high-energy physics, particle transport

$$
\begin{aligned}
& \text { Evaluate } \quad G=\iint_{a_{1} a_{2}}^{b_{1} b_{2}} \cdots \int_{a_{M}}^{b_{M}} g\left(r_{1}, r_{2}, \ldots, r_{M}\right) d r_{1} d r_{2} \ldots d r_{M} \\
& \text { where } r_{1}, r_{2}, \ldots, r_{M} \text { are all independent variables }
\end{aligned}
$$

For $k=1, \ldots, N$ :

For $m=1, \ldots, M: \quad$ choose $R_{m}^{(k)}$ randomly in $\left(a_{m}, b_{m}\right)$

$$
G \sim\left(b_{1}-a_{1}\right) \cdot \ldots \cdot\left(b_{M}-a_{M}\right) \cdot \frac{1}{N} \sum_{k=1}^{N} g\left(R_{1}^{(k)}, R_{2}^{(k)}, \ldots, R_{M}^{(k)}\right)
$$


Continuous Probability Density

- $f(x)$

- $0 \leq f(x)$

- Probability $\{a \leq x \leq b\}=\int_{a} f(x) d x$ $f(x)$

- Normalization:

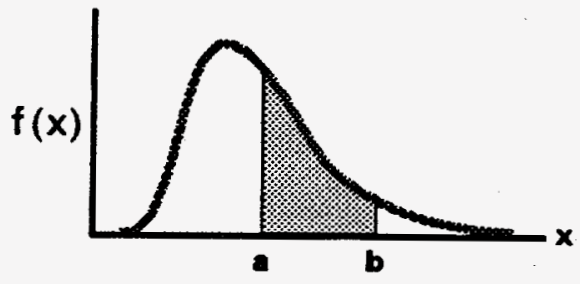

$$
\int_{-\infty}^{+\infty} f(x) d x=1
$$

Discrete Probability Density

$\bullet\left\{f_{k}\right\}, k=1, \ldots, N, \quad$ where $f_{k}=f\left(x_{k}\right)$

$-0 \leq f_{k}$

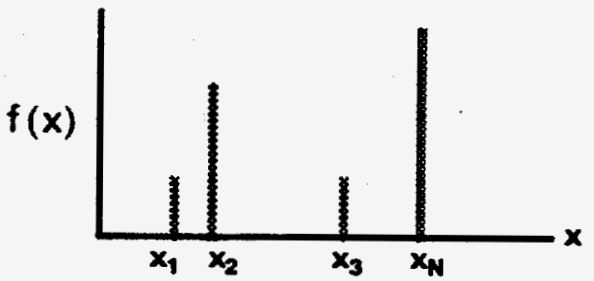

- Probability $\left\{x=x_{k}\right\}=f_{k}$

- Normalization:

$$
\sum_{k=1}^{N} f_{k}=1
$$

Introduction - Basic Statistics

- Mean, Average, Expected Value

$$
\begin{aligned}
x=\mu & =\langle x\rangle=E[x] \\
& +\infty \\
\mu & \int_{-\infty} x f(x) d x \quad \text { [continuous] } \quad \mu=\sum_{k=1}^{N} x_{k} f_{k} \quad \text { [discrete] }
\end{aligned}
$$

- Variance

$$
\begin{array}{ll}
\operatorname{var}(x)=\overline{(x-\mu)^{2}}=\sigma^{2}=\left\langle(x-\mu)^{2}\right\rangle=E\left[(x-\mu)^{2}\right] \\
\sigma^{2}=\int_{-\infty}^{+\infty}(x-\mu)^{2} f(x) d x & \sigma^{2}=\sum_{k=1}^{N}\left(x_{k}-\mu\right)^{2} f_{k}
\end{array}
$$

- Standard Deviation

$$
\sigma=\sqrt{\sigma^{2}}
$$

- Functions of a Random Variable

Consider $g(x)$, where $x$ is a random variable with density $f(x)$

$$
E[g(x)]=\int_{-\infty}^{+\infty} g(x) f(x) d x \quad E[g(x)]=\sum_{k=1}^{N} g_{k} f_{k}
$$


The key to Monte Carlo methods is the notion of random sampling.

- The problem can be stated this way:

Given a probability density, $f(x)$, produce a sequence of $\hat{x}$ 's.

The $\hat{x}$ 's should be distributed in the same manner as $f(x)$.

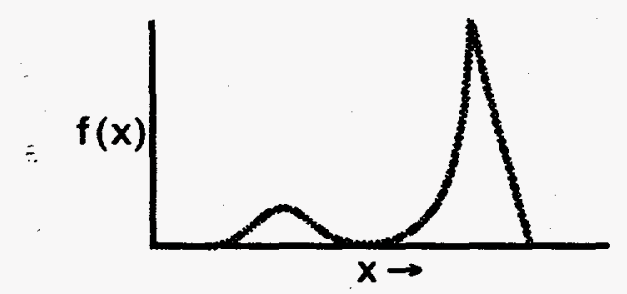

- The use of random sampling distinguishes Monte Carlo from all other methods

- When Monte Carlo is used to solve the integral Boltzmann transport equation

- Random sampling models the outcome of physical events

(e.g., neutron collisions, fission process, source, ..... )

- Computational geometry models the arrangement of materials 


\title{
Monte Carlo
}

\author{
\&
}

\section{Transport Equation}

Boltzmann Transport Equation - Time-Independent, Linear

$$
\Psi(r, v)=\int\left[\int \Psi\left(r^{\prime}, \mathbf{v}^{\prime}\right) C\left(\mathbf{v}^{\prime} \rightarrow \mathbf{v}, \mathbf{r}^{\prime}\right) d \mathbf{v}^{\prime}+Q\left(r^{\prime}, \mathbf{v}\right)\right] T\left(r^{\prime} \rightarrow r, v\right) d r^{\prime}
$$

where

- $\Psi(r, v)=$ particle collision density

- $Q\left(r^{\prime}, \mathbf{v}\right) \quad$ = source term

- $C\left(v^{\prime} \rightarrow \mathbf{v}, r^{\prime}\right)=$ collision kernel, change velocity at fixed position

- $T\left(r^{\prime} \rightarrow r, v\right)=$ transport kernel, change position at fixed velocity

- Angular Flux $\quad \Psi(\mathbf{r}, \mathbf{v})=\frac{\Psi(\mathbf{r}, \mathbf{v})}{\Sigma(\mathbf{r},|\mathbf{v}|)}$

- Scalar Flux

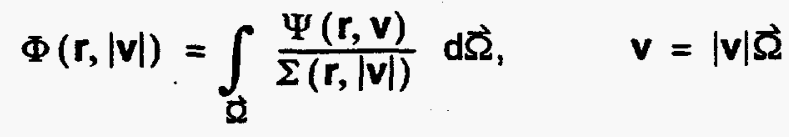


Source term for the Boltzmann equation

$$
Q(r, v)=\left(\begin{array}{ll}
S(r, v) & \Leftarrow \text { Fixed Source } \\
S(r, v)+\int \Psi\left(r, v^{\prime}\right) F\left(v^{\prime} \rightarrow v, r\right) d v^{\prime} & \Leftarrow \text { Fixed Source + Fission } \\
\frac{1}{k} \int \Psi\left(r, v^{\prime}\right) F\left(v^{\prime} \rightarrow v, r\right) d v^{\prime} & \Leftarrow \text { Eigenvalue }
\end{array}\right.
$$

where

$$
\begin{array}{lll}
-S(r, v) & \text { fixed source } \\
- & F\left(v^{\prime} \rightarrow \mathbf{v}, \mathbf{r}\right) & =\begin{array}{l}
\text { creation operator (due to fission), } \\
\text { particle at }\left(r, \mathbf{v}^{\prime}\right) \text { creates particle at }(r, v)
\end{array} \\
-K & =\text { eigenvalue }
\end{array}
$$

$$
\Psi(r, v)=\int\left[\int \Psi\left(r^{\prime}, v^{\prime}\right) C\left(v^{\prime} \rightarrow v, r^{\prime}\right) d v^{\prime}+Q\left(r^{\prime}, v\right)\right] T\left(r^{\prime} \rightarrow r, v\right) d r^{\prime}
$$

\section{Assumptions}

- static homogeneous medium

- time-independent

- Markovian - next event depends only on current $(r, v)$, not on previous events

- particles do not interact with each other

- neglect relativistic effects

- no long-range forces (particles fly in straight lines between events)

- material properties are not affected by particle reactions

- etc., etc.

$\rightarrow$ can use superposition principle 
Basis for Monte Carlo Solution Method

Let $\quad p=(r, v) \quad$ and $\quad R\left(p^{\prime} \rightarrow p\right)=C\left(v^{\prime} \rightarrow v, r^{\prime}\right) T\left(r^{\prime} \rightarrow r, v\right)$

Expand $\Psi$ into components having $0,1,2, \ldots, k$ collisions

$$
\Psi(p)=\sum_{k=0}^{\infty} \Psi_{k}(p), \quad \text { with } \Psi_{0}(p)=\int Q\left(r^{\prime}, v\right) T\left(r^{\prime} \rightarrow r, v\right) d r^{\prime}
$$

By definition,

$$
\Psi_{k}(p)=\int \Psi_{k-1}\left(p^{\prime}\right) R\left(p^{\prime} \rightarrow p\right) d p^{\prime}
$$

Note that collision $\mathbf{k}$ depends only on the results of collision $\mathbf{k}-1$, and not on any prior collisions $k-2, k-3, \ldots$

Statistical approach to determining $\Psi_{k}$

$$
\Psi_{k}(p)=\int \Psi_{k-1}\left(p^{\prime}\right) R\left(p^{\prime} \rightarrow p\right) d p^{\prime}
$$

- interpret terms in the following manner:

$$
\begin{gathered}
\Psi_{k-1}\left(p^{\prime}\right)=\text { probability density for occurence of }(k-1)^{\text {st }} \text { collisions at } p^{\prime} \\
R\left(p^{\prime} \rightarrow p\right)=\text { conditional probability that } a(k-1)^{\text {st }} \text { collision at } p^{\prime} \\
\text { will result in } a(k)^{t h} \text { collision at } p .
\end{gathered}
$$

- Monte Carlo method

(1) Randomly sample $p^{\prime}$ from $\Psi_{k-1}\left(p^{\prime}\right)$

(2) Given $p^{\prime}$, randomly sample $p$ from $R\left(p^{\prime} \rightarrow p\right)$

(3) If $p$ lies within $d p_{i}$ at $p_{i}$, tally 1 in bin $i$

$\rightarrow$ Repeat steps (1), (2), (3) $N$ times, then $\bar{\Psi}_{k}\left(p_{i}\right) d p_{i} \sim$ \{counts for bin $\left.i\right\} / N$ 


\section{Histories}

- After repeated substitution for $\Psi_{k}$

$$
\begin{aligned}
\Psi_{k}(p) & =\int \Psi_{k-1}\left(p^{\prime}\right) R\left(p^{\prime} \rightarrow p\right) d p^{\prime} \\
& =\int \ldots \int \Psi_{0}\left(p_{0}\right) R\left(p_{0} \rightarrow p_{1}\right) R\left(p_{1} \rightarrow p_{2}\right) \ldots R\left(p_{k-1} \rightarrow p\right) d p_{0} \ldots d p_{k-1}
\end{aligned}
$$

- A "history" is a sequence of states $\left(p_{0}, p_{1}, p_{2}, p_{3}, \ldots\right)$

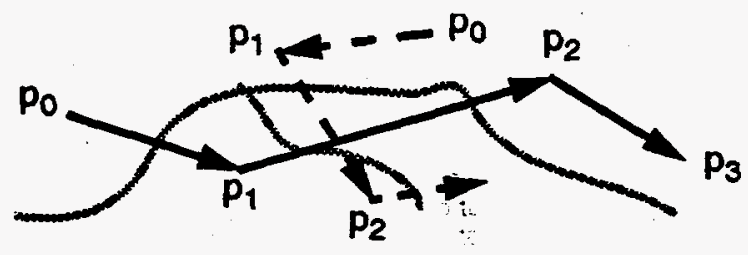

For estimates in a given region, tally the occurrences for each collision of each "history" within a region

$$
\Psi_{k}(p)=\int \cdots \int \Psi_{0}\left(p_{0}\right) R\left(p_{0} \rightarrow p_{1}\right) R\left(p_{1} \rightarrow p_{2}\right) \ldots R\left(p_{k-1} \rightarrow p\right) d p_{0} \ldots d p_{k-1}
$$

\section{Monte Carlo approach:}

- Generate a sequence of states, $\left(p_{0}, p_{1}, \ldots, p_{k}\right)$, [i.e., a history] by

- Randomly sample from PDF for source: $\quad \Psi_{0}\left(P_{0}\right)$

- Randomly sample from PDF for $k^{\text {th }}$ transition: $R\left(p_{k-1} \rightarrow p_{k}\right)$

- Generate estimates of results, by averaging over $\mathbf{M}$ histories:

$$
A=\int A(p) \Psi(p) d p=\frac{1}{M} \sum_{m=1}^{M}\left(\sum_{k=1}^{\infty} A\left(p_{k, m}\right)\right)
$$




\section{Monte Carlo}

\section{\&}

\section{Simulation}

"Simulation is better than reality"

Richard W. Hamming, 1991

Monte Carlo \& Simulation

Simulation approach to particle transport

- Faithfully simulate the history of a single particle from birth to death

During the particle history,

- model collisions using physics equations \& cross-section data

- model free-flight using computational geometry

- tally the occurrences of events in each region

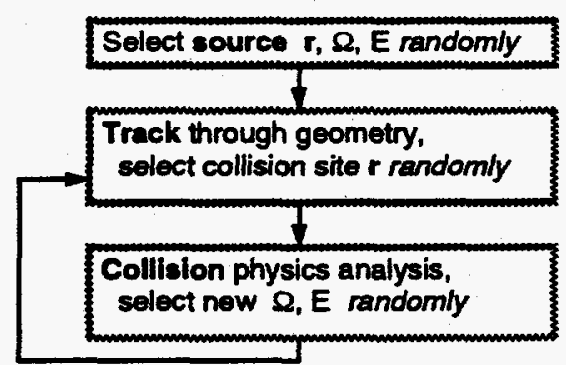

- Repeat the simulation for many histories, accumulating the tallies

- Fundamental rule:

Think like a neutron 


\section{Source}

- Random sampling

E, \& - analytic, discrete, or piecowise-tabulated PDF's

- Computational geometry

r - sample from region in 3-D space, or from discrete PDF

\section{Tracking}

\section{- Random sampling}

$d_{\text {collide }}$ - distance to collision, from mtp \& exponential PDF

- Computational geometry

$$
d_{\text {geom }} \text { - distance-to-boundary, ray-tracing, next-region, ..... }
$$

\section{Collisions}

- Random sampling

$$
E^{\prime}, \Omega^{\prime} \text { - analytic, discrete, or piecewise-tabulated PDF's }
$$

- Physics

$$
\Sigma, f(\mu) \text { - cross-section data, angular PDFs, kinematics, ..... }
$$

Tallies

- Statistics

Variance Reduction

- Random sampling

\section{Single particle}

- random-walk for particle history

- simulate events, from birth to death

- tally events of interest

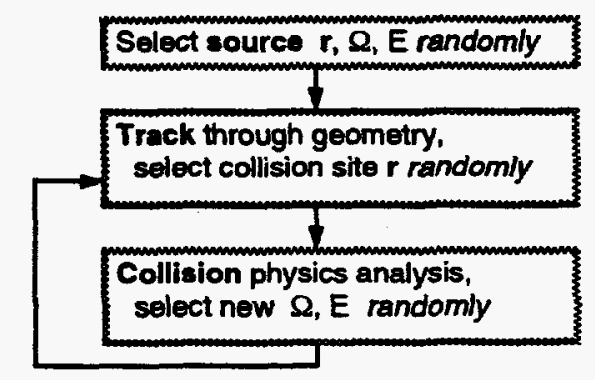

\section{Batch of histories ("generation")}

- random-walk for many particle histories

- tally the aggregate behavior

\section{Overall}

- timesteps

- geometry changes

- material changes

- fuel depletion

- bumable absorbers

- control rods 


\section{Random Number}

\section{Generation}

"Randomness is a negative property; it is the absence of any pattern."

Richard W. Hamming, 1991

"... random numbers should not be generated by a method chosen at random."

D. Knuth, $\sim 1981$

Random Number Generators

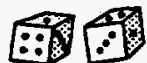

[24]

\section{Random Number Generators (RNGs)}

- Numbers are not random; a sequence of numbers can be.

- Truly random sequences are generally not desired on a computer.

- Pseudo-random sequences: - repeatable (deterministic)

- pass statistical tests for randomness

- RNG - function which generates a sequence of numbers which appear to have been randomly sampled from a uniform distribution on $(0,1)$.

- probability density for $f(x)$ :

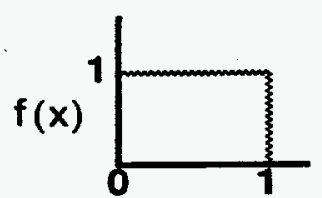

- typical usage in codes: $\quad x=\operatorname{ranf}()$

- also called "pseudo-random number generators" (PRNG)

- All other random sampling is performed using this basic RNG 
Most production-level Monte Carlo codes for particle transport

use linear congruential random number generators

$$
S_{i+1}=\left[S_{i} \cdot g+c\right] \bmod 2^{m}
$$

- robust, 40 years of heavy-duty use

- simple, fast

- theory is well-understood (e.g., DE Knuth, Vol 2, 177 pages)

- not the "best" generators, but good enough - RN's are used in unpredictable ways during particle simulation

- To achieve reproducibility of Monte Carlo calculations, despite vectorization or varying numbers of parallel processors, there must be a fast, direct method for skipping ahead (or back) in the random sequence.

Random Number Generators

\section{Linear Congruential PRNGs}

- due to Lehmer, 1949

- most common method, excellent (when not abused)

- Method:

$$
s_{0} \leftarrow \text { initial value }
$$

$$
\left[\begin{array}{l}
r_{k} \leftarrow s_{k} / p \\
s_{k+1} \leftarrow\left[g \cdot s_{k}+c\right] \bmod p
\end{array}\right.
$$

where

$s_{k}, g, c, p=$ integers, $\quad r_{k}=$ real

$$
\begin{aligned}
& s_{k}=\text { seed } \\
& \mathbf{g}=\text { generator, or multiplier } \\
& \mathbf{c}=\text { increment } \\
& \mathbf{p}=\text { modulus } \\
& \mathbf{r}_{\mathbf{k}}=\text { psuedo-random number, } 0 \leq r_{k} \leq 1
\end{aligned}
$$

- "mod p" $\Rightarrow \quad$ "remainder after division by p",

- Multiplicative: $\quad c=0$

$$
\text { - Mixed: } \quad c>0
$$


Example \#1: $\quad s_{k+1} \leftarrow\left[g \cdot s_{k}+c\right] \bmod p$

with $g=47, c=1, s_{0}=1, p=100$

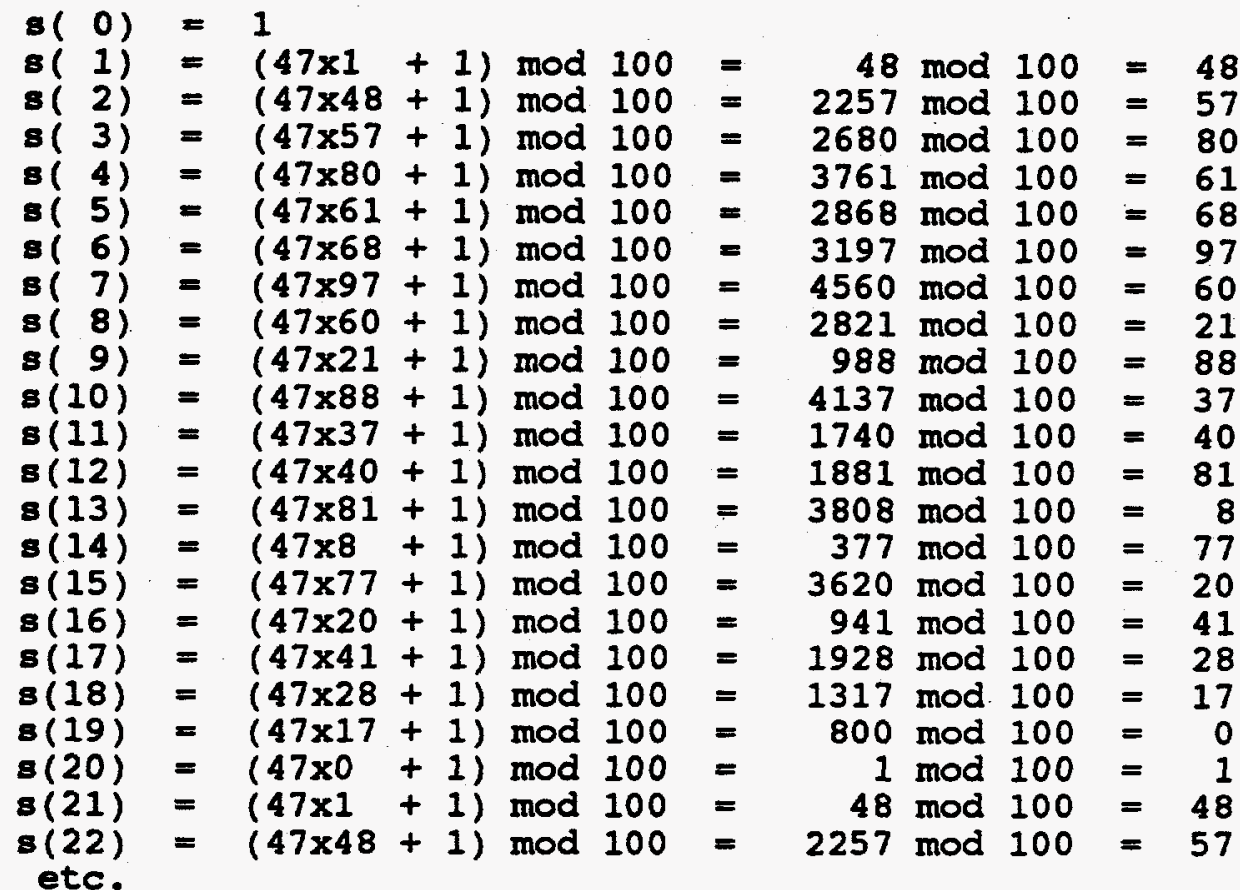

Random Number Generators

Example \#2:

$$
\begin{aligned}
& s_{k+1} \leftarrow\left[g \cdot s_{k}+c\right] \bmod p \\
& \text { with } g=5, c=1, s_{0}=1, p=100
\end{aligned}
$$

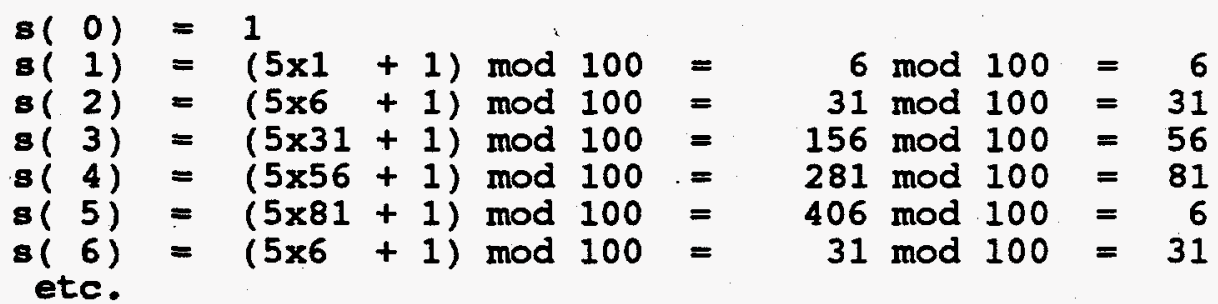

Example \#3:

$$
\begin{aligned}
& s_{k+1} \leftarrow\left[g \cdot s_{k}+c\right] \bmod p \\
& \quad \text { with } g=5, c=0, s_{0}=1, p=100
\end{aligned}
$$

$\mathbf{s}(0)=1$
$\mathbf{s}(1)=(5 \times 1) \bmod 100=5)$
$\mathbf{s}(2)=(5 \times 5) \bmod 100=25 \bmod 100=5$
$\mathbf{s}(3)=(5 \times 25) \bmod 100=125 \bmod 100=25$
$\mathbf{s}(4)=(5 \times 25) \bmod 100=125 \bmod 100=25$
etc.


Linear Congruential PRNG - Selecting g, c, p, so

$$
s_{k+1} \leftarrow\left\lfloor g \cdot s_{k}+c\right] \bmod p
$$

- For theoretical \& practical considerations in selecting $g, c, p, s_{0}$, see D. E. Knuth, The Art of Computer Programming. Vol 2

- Modulus (p):

- choose $p=2^{\mathrm{N}}$

- simplifies "mod $p$ "- discand all but the $N$ least significant bits

- simplifies division by $p$ - shift the "point" left by $N$ bits

- $N$ should be as large as possible, to maximize the period - $N>35$ is best.

- Usually, choose $\mathrm{N}$ to be number of bits in largest positive integer, for efficient computing

- Generator (g), Initial Seed (so), \& Increment (c) :

- choose $g$ \& $c$ to maximize the period

- large $g$ is best to reduce serial correlation

- Obviously, $g=1$ or $g=0$ are bad

- For $c=0$ (multiplicative PRNG):

choosing (1) $g \bmod 8=3$ or 5

(2) $s_{0}=$ odd

results in: period $=2^{N-2}$, the maximum possible period.

- For $c>0$ (mixed PRNG):

choosing (1) c relatively prime to $p$

(2) $(g-1)$ to be a multiple of every prime factor of $p$

(3) $\mathrm{g}-1)$ to be a multiple of 4 if $\mathrm{p}$ is a multiple of 4

results in: perlod $=2^{N}$, the maximum possible period.

Multiplicative congruential method - Lehmer

$$
\begin{array}{ll}
S_{i+1}=g \cdot S_{i}+c \bmod 2^{m}, & 0<S_{i}<2^{m} \\
\xi_{i}=S_{i} / 2^{m}, & 0<\xi<1
\end{array}
$$

Typical parameters

$\begin{array}{llllcc} & & 2^{m} & \text { period } & \boldsymbol{a} & \boldsymbol{\varepsilon} \\ \text { RACER } & \text { (KAPL) } & 2^{47} & 2^{45} & 84,000,335,758,957 & 0 \\ \text { RCP } & \text { (BAPL) } & 2^{48} & 2^{48} & 2^{9}+1 & 59,482,192,516,946 \\ \text { MORSE } & \text { (ORNL) } & 2^{47} & 2^{45} & 5^{15} & 0 \\ \text { MCNP } & \text { (LANL) } & 2^{48} & 2^{46} & 5^{19} & 0 \\ \text { VIM } & \text { (ANL) } & 2^{48} & 2^{46} & 5^{19} & 0 \\ \text { RANF } & \text { (CRAY) } & 2^{48} & 2^{46} & 44,485,709,377,909 & 0 \\ -\quad \text { (G. Marsaglia) } & 2^{32} & 2^{32} & 69069 & 1\end{array}$




\section{Aside ...}

For the multiplicative congruential method, why is the period limited to a maximum of $2^{\mathrm{N}-2}$ ??

$$
s_{k+1} \leftarrow g \cdot s_{k} \bmod p, \quad s_{0} \text { odd, } g \bmod 8=3 \text { or } 5
$$

- All $s_{k}$ 's are odd, $g$ is odd

$\Rightarrow \quad g \cdot s_{k}$ will always be odd, reduces period by a factor of two.

- For $g \bmod 8=3$, trailing bits of $g$ are (...011)

or

$g \cdot s_{k}=(\ldots 011) \cdot(\ldots . .11)=(. .11)$

$g \cdot s_{k}=(\ldots 011) \cdot(\ldots .01)=(\ldots .01)$

$\Rightarrow$ next-to-last bit of $s_{k}$ will not change, reduces period by a factor of two.

- For $g \bmod 8=5$, trailing bits of $g$ are $(. . .101)$

or $g \cdot s_{k}=(. .101) \cdot(. .1 \times 1)=(. .1 \times 1)$

$g \cdot s_{k}=(. .101) \cdot(. .0 \times 1)=(. .0 \times 1)$

$\Rightarrow$ third-to-last bit of $s_{k}$ will not change, reduces period by a factor of two.

Example - CYBER-205 RANF

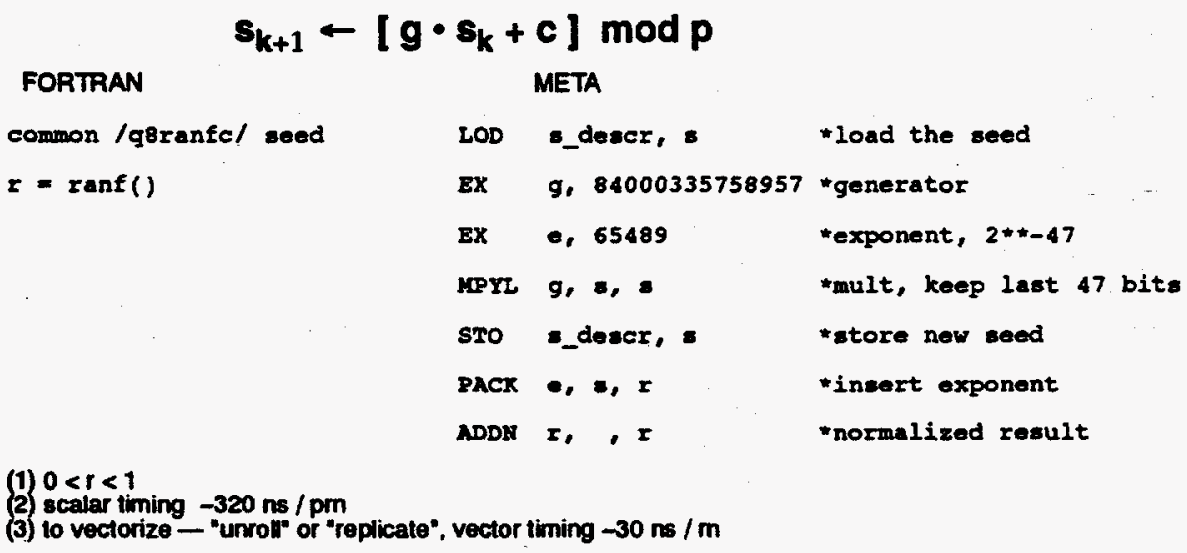

How long will the PRNs last?

$\begin{array}{ll}\text { Sharp EL-5155 } & \\ \text { CYBER-205, } & \text { scalar } \\ \text { CRAY-1, } & \text { vector } \\ \text { CYBER-205, } & \text { 2-pipe vector } \\ \text { CYBER-205, } & \text { 4-pipe vector } \\ \text { CRAY-XMP/48, vector x } 4 \\ \text { CRAY-2, } & \text { vector x } \\ \text { ETA-10, } & \text { vector X8 } \\ \text { CraY-C90, } & \text { vector X } 16\end{array}$

IIme to generate ALL2 $2^{45}$ RNs

$1 \mathrm{Myr}$
4 mos
15 days
12 days
6 days
$3 \mathrm{days}$
$30 \mathrm{hr}$
$13 \mathrm{hr}$
$4 \mathrm{hr}$




\section{Other PRNGs}

- Middle-square method:

- Quadratic-congruential:

- Modified Middle-square:

- Additive:

- Additive (or Shift):

- Ceneralized Additive (or Shift):

- Quasi-random sequences ....

- etc., etc., .....

\section{Testing PRNGs}

- See Knuth, Vol. 2, pp. 38-113

- Empirical Tests:

\section{Chi-square test} Serial pair, triplet, .... distributions Coupon Collector test

Collision test Gap test Run test etc.

Serial Correlation coefficients

- Theoretical Tests

Spectral test

Serial Correlation (global)

$$
\begin{aligned}
& s_{k+1}=\text { middle digits of } s_{k}^{2} \\
& s_{k+1}=\left[a \cdot s_{k}^{2}+b s_{k}+c\right] \bmod p \\
& s_{k+1}=\left[s_{k} \cdot\left(s_{k}+1\right)\right] \bmod p \\
& s_{k+1}=\left[s_{k}+s_{k-i}\right] \bmod p \\
& s_{k}=\left[s_{k-j}+s_{k-i}\right] \bmod p \\
& s_{k}=\left[a_{1} s_{k-1}+a_{2} s_{k-2} \ldots a_{k} s_{k-1}\right] \bmod p
\end{aligned}
$$

Spectral test

ibility

Random Number Generators - Reproducibllity

Frequency test Poker tests Maximum-of-t test

etc.

\section{Reproducibility of a Particle History}

- use separate, distinct random sequence for each particle

- starting seeds for separate particles are separated by "stride"

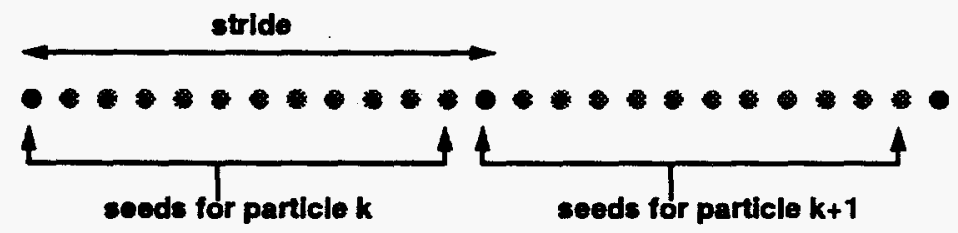

- stride should be large enough to prevent overlap (for most histories)

-1000 is common for reactor analysis problems

- splitting \& variance reduction not needed for in-core physics

- reduces total random number usage

$-4,297$ is the "old" default for MCNP \& VIM

$-152,917$ is the default for MCNP \& VIM

- prepared for lots of splitting \& variance reduction - potential for lots of secondary particles 
Parallel processing

- take "super-stride" in random sequence for particles on each processor

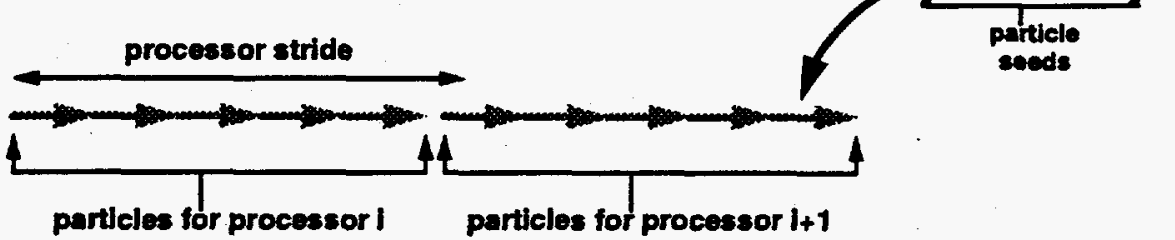

\section{Eigenvalue Problems}

- batches of particles, distributed among parallel processors

- seeds for each

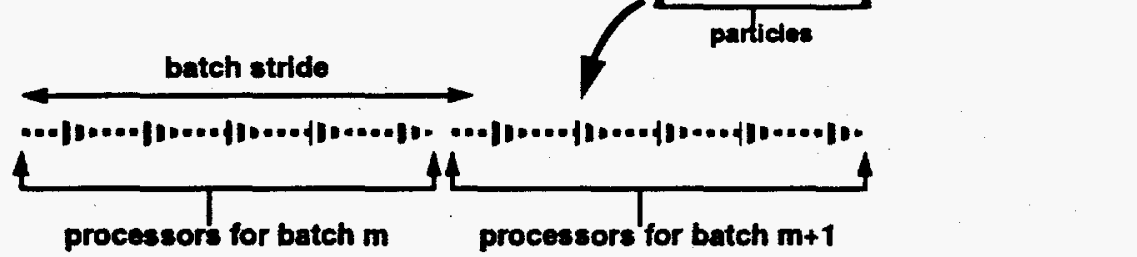

To skip ahead $k$ steps in the random sequence, [initial seed] $\rightarrow\left[k^{\text {th }}\right.$ seed]

$$
\begin{aligned}
S_{k} & =g \cdot S_{k-1}+c \bmod 2^{m} \\
& =g \cdot\left(g \cdot S_{k-2}+c\right)+c \bmod 2^{m} \\
& \left.=g\left(\ldots . g\left(g\left(g S_{0}+c\right)+c\right)+c\right) \ldots \ldots\right)+c \bmod 2^{m} \\
& =g^{k} \cdot S_{0}+c \cdot\left(g^{k-1}+g^{k-2}+\ldots . .+g+1\right) \bmod 2^{m} \\
& =g^{k} \cdot S_{0}+c \cdot\left(g^{k}-1\right) /(g-1) \bmod 2^{m}
\end{aligned}
$$

- Periodic sequence:

negative skip $\boldsymbol{k}_{n}$ equivalent to positive skip (period $-\boldsymbol{k}_{n}$ )

- Can skip from any seed directly to any other:

initial seed $\rightarrow t^{\text {th }}$ seed for $F^{\text {th }}$ particle on $m^{\text {th }}$ processor in $n^{\text {th }}$ batch particle $i \rightarrow$ particle $j$

batch $i \rightarrow$ batch $j$

- All arithmetic must be performed $\bmod 2^{m}$, without truncation or roundoff

$$
S_{k}=G(k) \cdot S_{0}+C(k) \bmod 2^{m}
$$


Define $\quad G(k)=g^{k} \bmod 2^{m}$

$$
\begin{array}{ll}
m=32 \text { or } 48 \text { (typical), } & \text { based on the size of a computer word } \\
-2^{m}<k<+2^{m}, & \text { based on desired "stride" }
\end{array}
$$

Denote the $j^{\text {th }}$ bit of $k$ by $k_{[j]}$, so that

$$
k=2^{m-1} k_{[m-1]}+2^{m-2} k_{[m-2]}+\ldots . .+2^{1} k_{[1]}+2^{0} k_{[0]}
$$

Substituting into $G(k)$ yields

$$
\begin{aligned}
G(k) & =g^{k} \bmod 2^{m}=g^{\sum_{i=0}^{m-1} k_{[1]^{2}}} \bmod 2^{m} \\
& =\prod_{j=0}^{m-1}\left(g^{2^{j}}\right)^{k}[i] \bmod 2^{m}
\end{aligned}
$$

Efficient algorithms for evaluating $G(k)$ can be formulated using only $m$ steps

Random Number Generators - Skip Ahead - Computing G(k)

Enumerating a few terms of $G(k)$ makes the algorithm obvious

$$
G(k)=\left(g^{1}\right)^{k[0]} \cdot\left(g^{2}\right)^{k}[1] \cdot\left(g^{4}\right)^{k}[2] \cdot\left(g^{8}\right)^{k[3]} \cdots\left(g^{2^{m-1}}\right)^{k}[m-1] \bmod 2^{m}
$$

Note that $k_{[]}=0$ or $k_{0]}=1$, so that each term $\left(g^{n}\right)^{k}[i]$ evaluates to either 1 or $g^{n}$.

\section{Algorithm G:}

$$
\begin{aligned}
& G \leftarrow 1, \quad h \leftarrow g, \quad i \leftarrow k+2^{m} \bmod 2^{m} \\
& \text { while } i>0 \\
& \quad \text { if } \quad i=\text { odd: } \quad G \leftarrow G h \bmod 2^{m} \\
& h \leftarrow h^{2} \bmod 2^{m} \\
& \quad i \leftarrow\lfloor i / 2\rfloor
\end{aligned}
$$

\section{Remarks}

- Algorithm $\mathbf{G}$ terminates after $m$ steps, rather than $\mathbf{k}$ steps

- Negative strides are trivial, due to periodicity: $\quad G(-s)=G\left(2^{m}-s\right)$ 
Define

$$
\begin{aligned}
C(k) & =c\left(\frac{g^{k}-1}{g-1}\right) \bmod 2^{m} \\
& =c \cdot\left(1+g+g^{2}+g^{3}+\ldots+g^{k-1}\right) \bmod 2^{m}
\end{aligned}
$$

The series for $C(k)$ can be evaluated recursively, similar to $G(k)$, in $m$ steps:

\section{Algorithm C:}

$$
\begin{array}{ll}
c \leftarrow 0, & f \leftarrow c, \quad h \leftarrow g, \quad i \leftarrow k+2^{m} \bmod 2^{m} \\
\text { while } & i>0 \\
& \text { if } i=\text { odd: } \quad c \leftarrow c h+f \bmod 2^{m} \\
& f \leftarrow f(h+1) \bmod 2^{m} \\
& h \leftarrow h^{2} \bmod 2^{m} \\
& i \leftarrow\lfloor i / 2\rfloor
\end{array}
$$

- Since most of the common random number generators use $c=0$, Algorithm $\mathbf{C}$ is generally not required.

- Algorithm C can be included with Algorithm G, at very little extra cost

Random Number Generators - Examples - Computer Coding

\section{Computer Coding}

- All integer adds \& multiplies must be performed exactly $\left(\bmod 2^{m}\right)$, without truncation or roundoff

- For $\mathrm{m} \leq 32$, reasonably portable coding is straightforward in C

- For $m>32$, or for Fortran coding

- split integers into "high" \& "low" pieces

- perform modular arithmetic - straightforward (but tedious)

$\Rightarrow$ see Hendricks, Trans ANS 62, 283, 1990 -

same techniques can be used for arbitrary skips

- For Fortran, reasonably portable coding, except:

-Sparc2, rs6000, indigo, ...: "double precision"

- Cray: "real" 
R. N. Generator for 32-bit machines (sparc2, rs6000, indigo, .....)

$s \leftarrow 69069 \cdot s+1 \bmod 2^{32}$

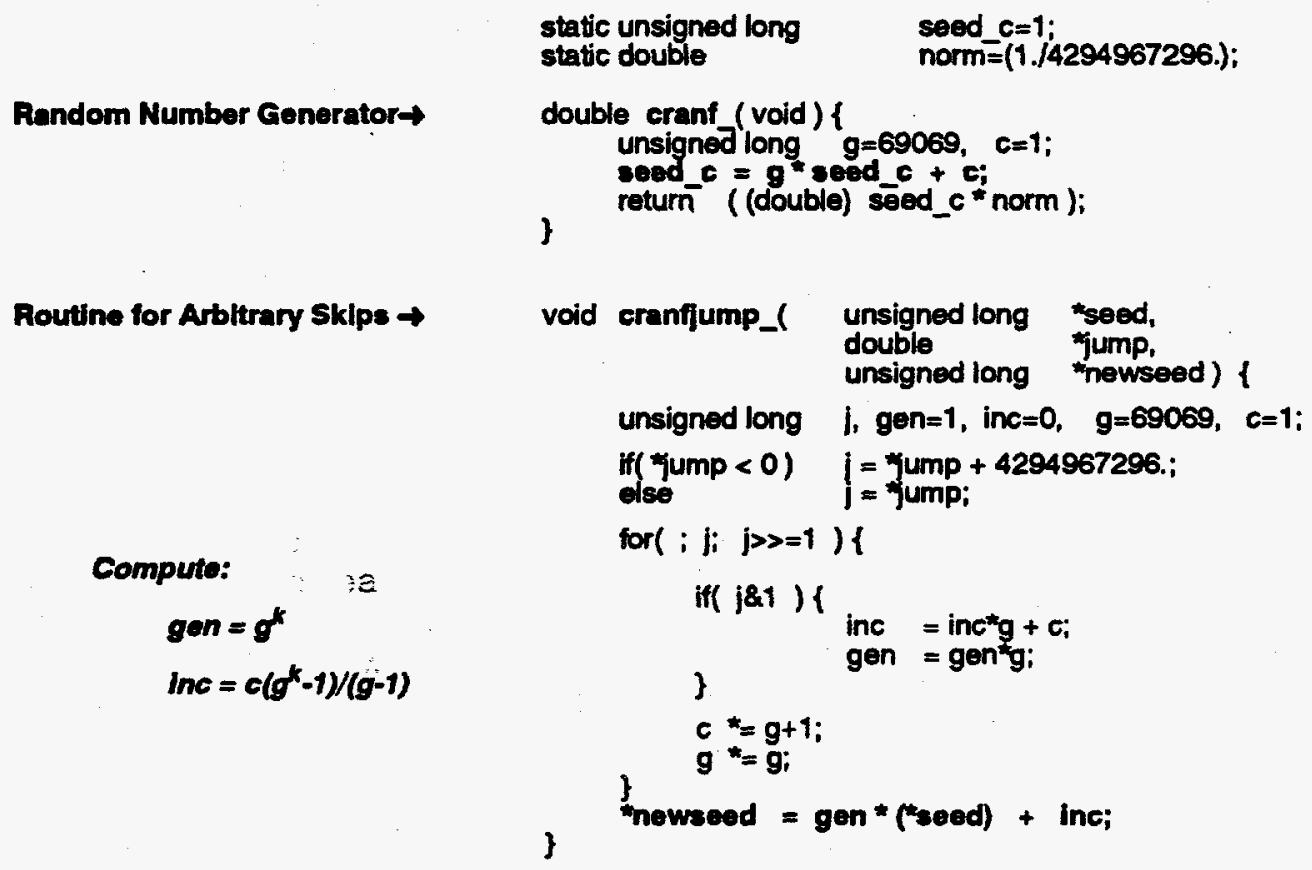

Random Number Generator - Examples - Tosting \& Timing

Fortran, 48-bit generator: $\quad g=5^{19}, \quad c=0, \quad m=48 \quad$ (NIM \& MCNP)

C, 32-bit generator: $\quad g=69069, \quad c=1, \quad m=32 \quad$ (from Marsaglia)

C, 32-bit

Sparce rs6000/350

random number

skip forward, average for $+1 \ldots 10^{5}$

$1.0 \mu s \quad .7 \mu s$

skip backward, average for $-1 \ldots-10^{5}$

$7.4 \mu \mathrm{s} \quad 10 \mu \mathrm{s}$

$4.0 \mu s \quad 20 \mu s$

Fortran, 48-bit

random number

skip forward, $\quad+152,917$

skip backward, $\quad-152,917$

skip forward, average for $+1 \ldots 10^{5}$

skip backward, average for $-1 \ldots-10^{5}$

$3.6 \mu \mathrm{s} \quad 2.3 \mu \mathrm{s}$

skip forward, $\quad+1,152,917$

skip forward, $\quad+1,152,917$, brute force

$163 \mu \mathrm{s} \quad 78 \mu \mathrm{s}$

$458 \mu \mathrm{s} \quad 215 \mu \mathrm{s}$

$160 \mu \mathrm{s} \quad 75 \mu \mathrm{s}$

$695 \mu \mathrm{s} \quad 232 \mu \mathrm{s}$

skip backward, $\quad-1,152,917$

skip backward, -1,152,917, brute force

$189 \mu \mathrm{s} \quad 90 \mu \mathrm{s}$

$4.1 \mathrm{sec} \quad 2.6 \mathrm{sec}$

$456 \mu s \quad 210 \mu s$

8 year 5 year 
- Algorithms for direct skip-ahead in the random sequence are simple, fast, convenient, ......, for modern Monte Carlo codes

- Arbitrary positive or negative strides can be taken, without precomputing or hardwiring specific constants

- Direct skip-ahead simplifies the initialization of random numbers for each particle, especially for parallel processing

- Algorithms described are currently used in:

$$
\begin{array}{lll}
\text { parallel VM } & \text { - ANL } & \text { - Sun, rs6000, SP1, .... } \\
\text { RACER } & \text { - KAPL } & \text { - Cray, Meiko CS1 \& CS2, Sun, SGI, ..... } \\
\text { KENO-Va } & - \text { CSN (Spain) } & \text { - Convex-C3440 }
\end{array}
$$




\section{Random}

\section{Sampling}

"Anyone who considers arithmetical methods of producing random digits is, of course, in a state of $\sin$."

J. Von Neumann, 1951

Monta Carto \& Random Sampling

Probability Density Function (PDF)

- $f(x)$

- $0 \leq f(x)$

- Probability $\{a \leq x \leq b\}=\int_{a} f(x) d x$

- Normalization:

$$
\int_{-\infty}^{+\infty} f(x) d x=1
$$

\section{Cumulative Distribution Function (CDF)}

$$
\begin{aligned}
& \text { - } F(x)=\int_{-\infty}^{x} f\left(x^{\prime}\right) d x^{\prime} \\
& \text { - } 0 \leq F(x) \leq 1 \\
& \text { - } 0 \leq \frac{d}{d x} F(x) \\
& \text { - } F(-\infty)=0, \quad F(\infty)=1
\end{aligned}
$$
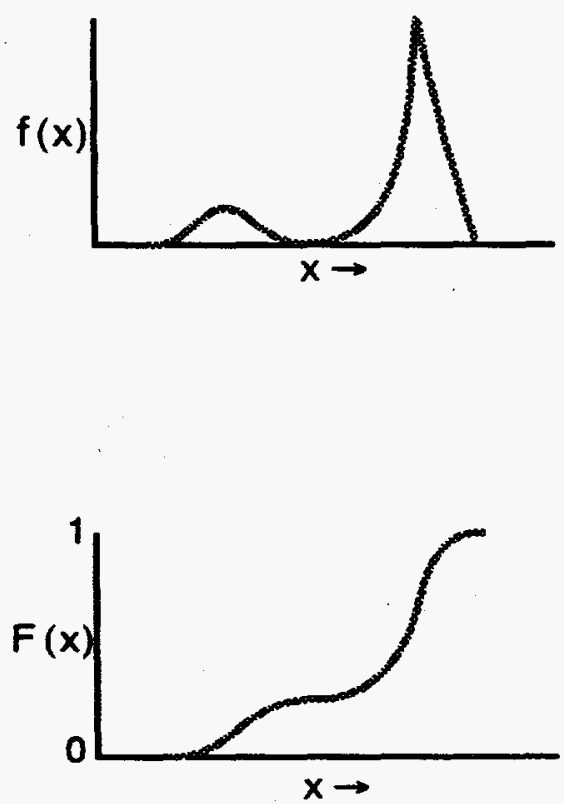


\section{Monte Carlo Codes}

\section{Categories of Random Sampling}

- Random number generator $\rightarrow$ uniform PDF on $(0,1)$

- Sampling from analytic PDF's $\rightarrow$ normal, exponential, Maxwellian, .....

- Sampling from tabulated PDF's $\rightarrow$ angular PDF's, spectrum, .....

\section{For Monte Carlo Codes...}

- Uniform random numbers, $\xi$, are produced by the R.N. generator on $(0,1)$

- Non-uniform random variates are produced from the $\xi$ 's by

- direct inversion

- rejection methods

- transformations

- composition (mixtures)

- sums, products, ratios, .....

- table lookup + interpolation

- lots (!) of other tricks .....

$-<10 \%$ of total cpu time (typical)

\section{Pseudo-Random Numbers}

- Not strictly "random", but good enough

- pass statistical tests

- reproducible sequence

- Uniform PDF on $(0,1)$

- Must be easy to compute

Multiplicative congruential method

- Algorithm

$$
\begin{aligned}
& S_{0}=\text { initial seed, odd integer, }<M \\
& S_{k}=G \cdot S_{k-1} \bmod M, \quad k=1,2, \ldots . \\
& \xi_{k}=S_{k} / M
\end{aligned}
$$

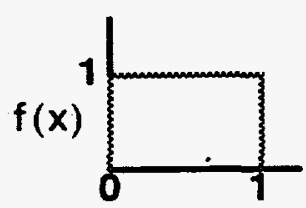

\section{Usage}

- In algorithms, usually denote RN uniform on $(0,1)$ by $\xi$

- In codes, invoke basic RN generator: . r ranfo

- Each new usage of $\xi$ or ranf0 generates a new RN 


\section{Direct Sampling}

Direct Solution of

$$
\begin{array}{lc}
\hat{x} \leftarrow F^{-1}(\xi) & \hat{x} \\
\text { Solve for } \hat{x}: & \xi=\int_{-\infty} f\left(x^{\prime}\right) d x^{\prime}
\end{array}
$$

Sampling Procedure:

- Generate $\xi$

- Determine $\hat{x}$ such that $F(\hat{x})=\xi$

\section{Advantages}

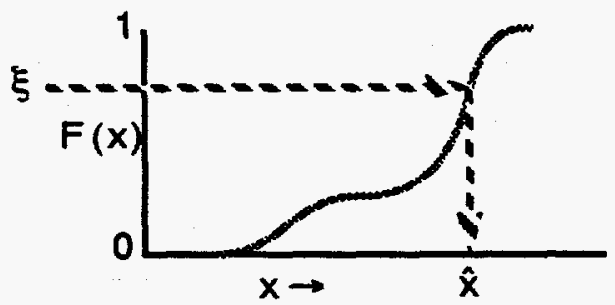

- Straightforward mathematics \& coding

- "High-level" approach

Disadvantages

- Often involves complicated functions

- In some cases, $F(x)$ cannot be inverted (e.g., Klein-Nishina)

\section{Rejection Sampling}

\section{Von Neumann}

$$
\begin{aligned}
& \text { ".... it seems objectionable to compute a } \\
& \text { transcendental function of a random number." }
\end{aligned}
$$

Select a bounding function, $g(x)$, such that

- $c \cdot g(x) \geq f(x)$ for all $x$

- $g(x)$ is an easy-to-sample PDF

Sampling Procedure:

- sample $\hat{x}$ from $g(x): \quad \hat{x} \leftarrow G^{-1}\left(\xi_{1}\right)$

- test: $\xi_{2} \cdot \operatorname{cg}(\hat{x}) \leq f(\hat{x})$

$$
\begin{aligned}
& \text { if true } \rightarrow \text { accept } \hat{x} \text {, done } \\
& \text { if false } \rightarrow \text { reject } \hat{x} \text {, try again }
\end{aligned}
$$

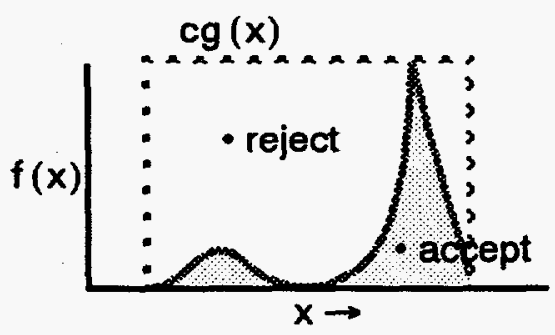

Efficiency

$\eta=\%$ of trials accepted $=\int f(x) d x / \int \operatorname{cg}(x) d x$

\section{Advantages}

- Simple computer operations

\section{Disadvantages}

- "Low-level" approach, sometimes hard to understand

- Will be inefficient if $\mathrm{cg}(x)$ and $f(x)$ do not have "similar" shapes \& ranges 


\section{Discrete PDF's}

- Discrete PDF

$\left\{f_{k}\right\}$, where $f_{k}=f\left(x_{k}\right), \quad k=1, \ldots, N$

$$
\begin{aligned}
& f_{k} \geq 0 \\
& \sum_{j=1}^{N} f_{j}=1
\end{aligned}
$$

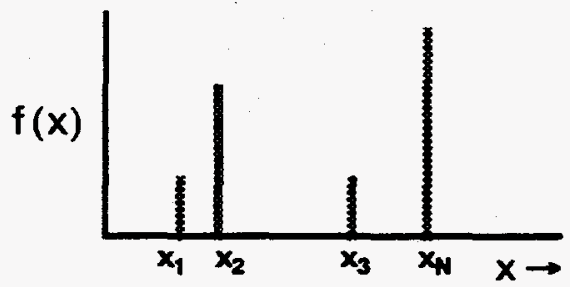

- Discrete CDF

$$
\begin{array}{r}
\left\{F_{k}\right\}, \text { where } F_{k}=\sum_{j=1}^{k} f_{j} \\
\text { and } \begin{array}{r}
F_{0}=0 \\
F_{N}=1
\end{array}
\end{array}
$$

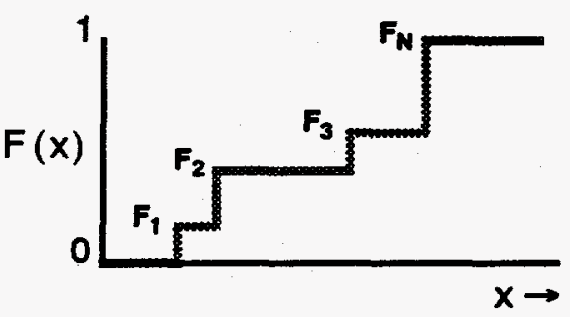

$\boldsymbol{F}_{1}$

Random Sampling - Discrete PDFs

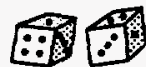

[52]

Sampling from Discrete PDF's - Conventional Procedure

Direct Solution of $\hat{\mathrm{x}} \leftarrow \mathrm{F}^{-1}(\xi)$

(1) Generate $\xi$

(2) Determine $k$ such that $F_{k-1} \leq \xi \leq F_{k}$

(3) Return $\hat{x}=x_{k}$

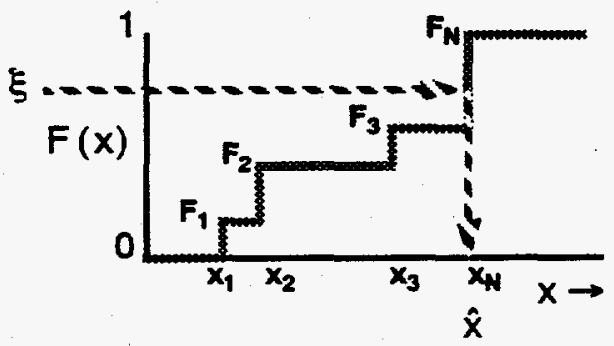

Step (2) requires a table search

- linear table searches require $O(N)$ time

- use when $\mathbf{N}$ small

- binary table searches require $O\left(\ln _{2} N\right)$ time

- use when $\mathbf{N}$ large

For some discrete PDFs, $F_{k}$ 's are not precomputed.

- linear search, with $F_{k}$ 's computed on-the-fly as needed 
Example - Sampling from Discrete Uniform PDF

Discrete Uniform PDF

$$
\begin{array}{ll}
f_{k}=1 / N, & k=1, \ldots, N \\
F_{k}=k / N, & F_{0}=0, F_{N}=1
\end{array}
$$

Sampling procedure:

Could use table search method, ....
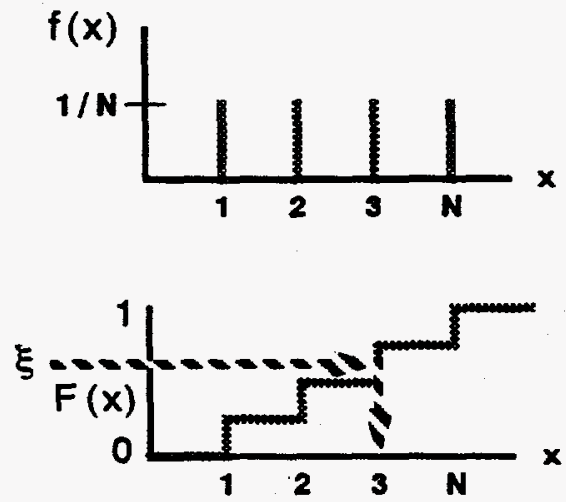

Easier, for this special case:

$$
\begin{aligned}
& K \leftarrow\lfloor 1+N \xi\rfloor, \quad \text { where }\lfloor y\rfloor \text { is the "floor" function, } \\
& \text { largest integer }<y
\end{aligned}
$$

Note: must be sure that $\lfloor 1+N \xi\rfloor \leq N$

Random Sampling - Discrote PDFs

\section{Examples — Sampling from Discrete PDFs}

\section{Multigroup scattering}

- inelastic scatter from MUFT-group $\mathbf{g}$ to MUFT-group g'

- thermal scatter from THAV-group g to THAV-group g'

$$
f_{g^{\prime}}=\frac{\sigma_{g \rightarrow g^{\prime}}}{\sum_{k} \sigma_{g \rightarrow k}}, \quad \text { where } \begin{aligned}
& g^{\prime}=1, \ldots, G_{\text {THAV }} \text { for thermal, } \\
& g^{\prime}=g, \ldots, G_{\text {MUFT }} \text { for inelastic, } \\
& k=1, \ldots, G_{\text {THAV }} \text { for thermal, } \\
& k=g, \ldots, G_{\text {MUFT }} \text { for inelastic }
\end{aligned}
$$

Selection of scattering nuclide for a collision

$$
f_{k}=\frac{N^{(k)} \sigma_{s}^{(k)}}{\sum_{k^{\prime}=1}^{K} N^{\left(k^{\prime}\right)} \sigma_{s}^{(k)}} \quad \text { where } \begin{aligned}
& k=1, \ldots, k \\
& K=\# \text { nuclides in composition }
\end{aligned}
$$


Sampling from Discrete PDF's - Allas Method

Any discrete PDF can be converted into "Alias sampling" form

original PDF: $\quad\left\{f_{k}\right\}, \quad k=1, \ldots, N$

where $f_{k}=$ probability of selecting $x=x_{k}$

aliased PDF: $\quad\left\{q_{k}, i_{k},\right\}, \quad k=1, \ldots, N$

$$
\text { where } \begin{array}{ll}
\frac{1}{N} \cdot q_{k} & =\text { prob. of selecting } \hat{x}=x_{k} \\
\frac{1}{N} \cdot\left(1-q_{k}\right) & =\text { prob. of selecting } \hat{x}=x_{i_{k}}
\end{array}
$$

Alias sampling procedure:

Select uniformly for $\hat{k}$ :

$$
\hat{k} \leftarrow\left\lfloor 1+N \xi_{1}\right\rfloor
$$

Select either $\hat{\mathbf{k}}$ or its "alias" $\mathbf{i}_{\hat{\mathbf{k}}}$ :

if $\xi_{2}<q_{\hat{k}}, \quad \hat{x} \leftarrow x_{\hat{k}}$,

otherwise, $\quad \hat{x} \leftarrow x_{i_{\hat{k}}}$

.....(continued on next page)

\section{Sampling from Discrete PDF's - Alias Method (continued)}

Why bother with "alias sampling" ?

$\rightarrow$ No table search needed, requires $O(1)$ time

$\rightarrow$ Sampling time is constant $\&$ independent of slze of PDF

$\rightarrow$ Vectorizes completely \& efficiently

$\rightarrow$ Fastest possible way to sample discrete PDFs

$\rightarrow$ Invented by Brown (who later found out Walker did it 3 yr earlier)

Creating the "aliased PDF" amounts to converting an N-way tree from arbltrary branching probabilities with single outcomes

to unlform branching probabilities with dual outcomes

(See FB Brown \& RACER coding for the set up algorithm)
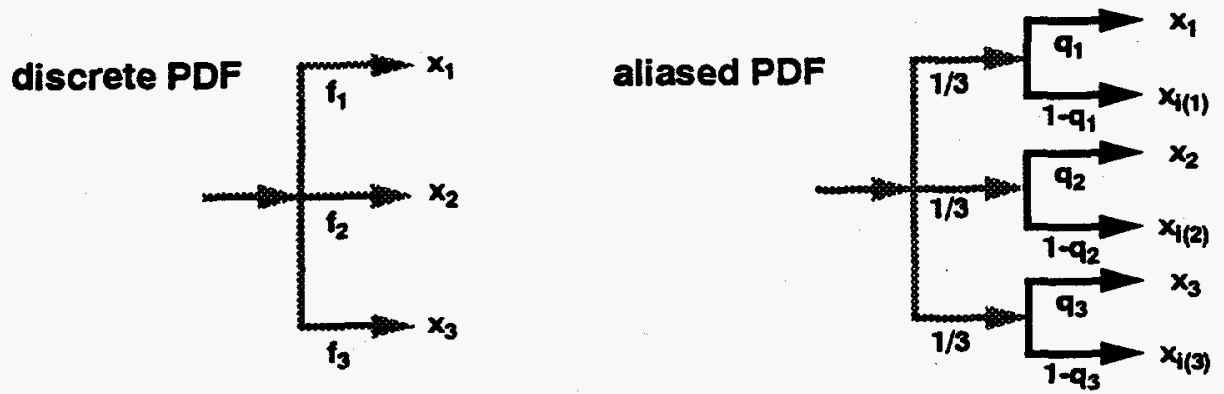
Examples - Sampling from "Histogram" PDFs

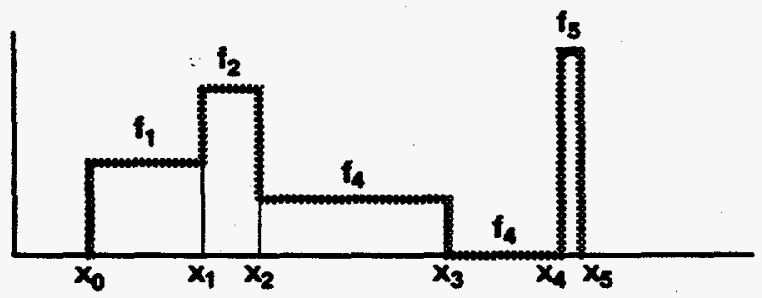

Two-step sampling: (1) Sample from discrete PDF to select a bin (2) Sample from uniform PDF within bin

- Discrete PDF: $\quad p_{k}=f_{k} \cdot\left(x_{k}-x_{k-1}\right), \quad k=1, \ldots, N$

- generate $\xi$

- use table search or alias method to select $K$

- Uniform sampling within bin $\mathrm{K}$ :

- generate $\xi$

- then,

$$
\hat{x} \leftarrow x_{K-1}+\left(x_{K}-x_{K-1}\right) \xi
$$

Random Sampling - Continuous PDFs

Examples - Sampling from Linear PDF on $(0,1)$

$$
\begin{aligned}
& f(x)=2 x, \quad 0 \leq x \leq 1 \\
& F(x)=\int_{0}^{x} f\left(x^{\prime}\right) d x^{\prime}=\int_{0}^{x} 2 x^{\prime} d x^{\prime}=x^{2}
\end{aligned}
$$

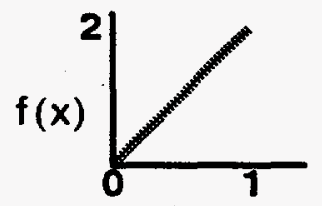

Direct Sampling:

$$
\text { solving } F(\hat{x})=\xi \text { or } \hat{x} \leftarrow F^{-1}(\xi)
$$

gives:

$$
\hat{\mathbf{x}} \leftarrow \sqrt{\xi}
$$

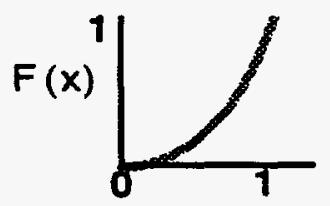

Examples - Sampling from $x^{n}$ PDF on $(0,1)$

$$
\begin{aligned}
& f(x)=(n+1) x^{n}, \quad 0 \leq x \leq 1 \\
& F(x)=x^{n+1}
\end{aligned}
$$

Solving $F(\hat{x})=\xi$ gives: $\quad \hat{x} \leftarrow \xi^{\frac{1}{n+1}}$ 
Examples - Sampling from Arbitrary Linear PDF

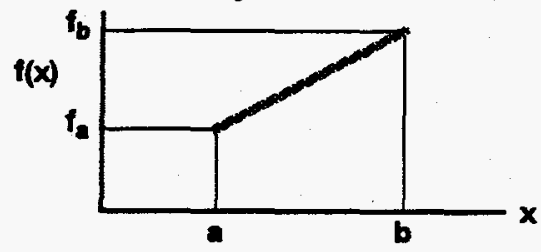

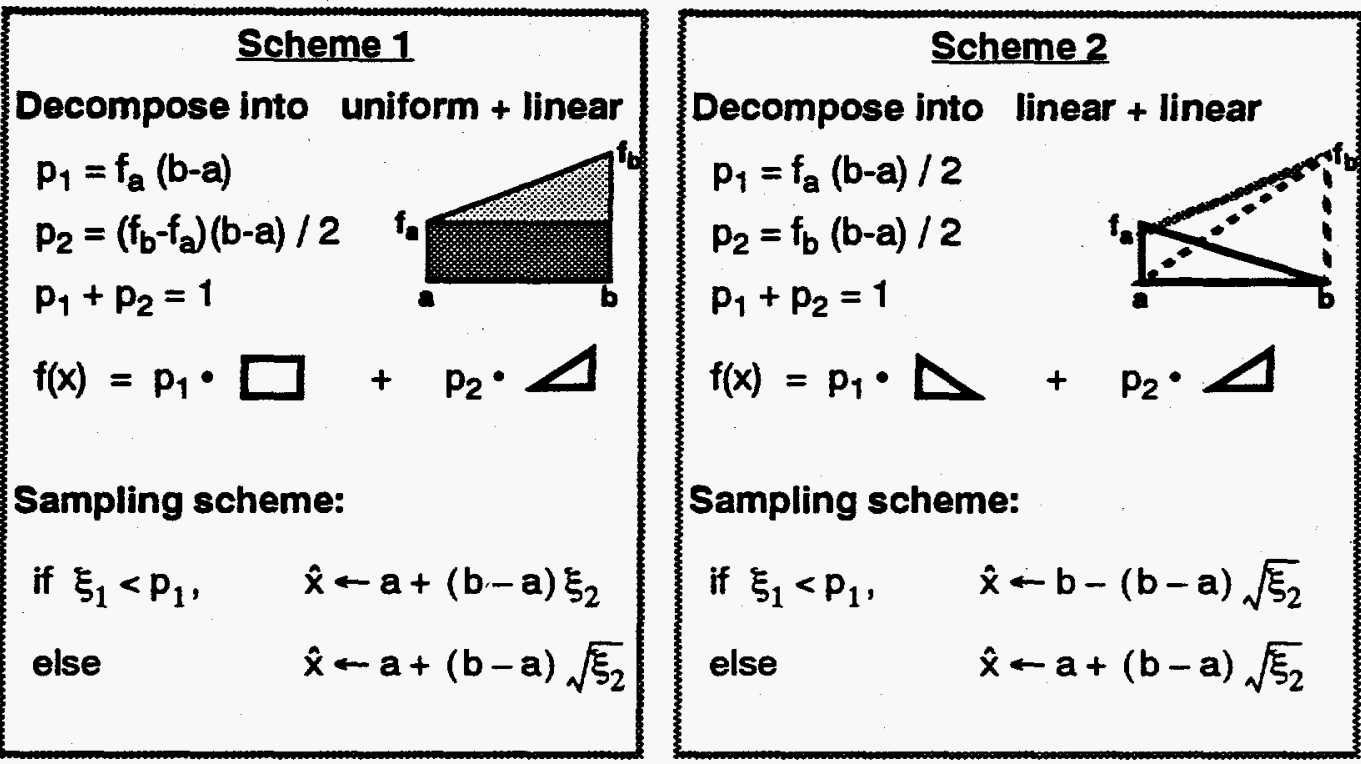

Random Sampling - Continuous PDFs

\section{Examples - Sampling from Piecewise Linear PDF} $t_{5}$

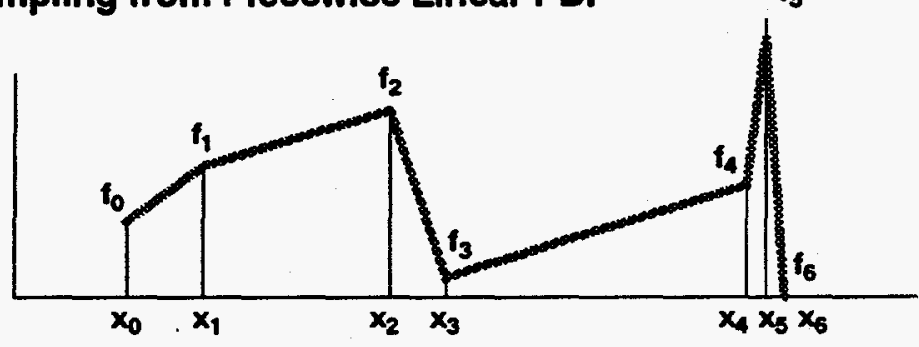

\section{Two-step sampling: $\quad$ (1) Sample from discrete PDF to select a bin} (2) Sample from linear PDF within bin

- Discrete PDF: $\quad p_{k}=\frac{\left(f_{k}+f_{k-1}\right)}{2} \cdot\left(x_{k}-x_{k-1}\right), \quad k=1, \ldots, N$

- generate $\xi$

- use table search or alias method to select $\mathbf{K}$

- Linear sampling within bin $\mathrm{K}$ :

- generate $\xi$

- then,

$$
\begin{array}{ll}
\text { if } \quad \xi_{1}<\frac{f_{k-1}}{f_{k}+f_{k-1}}, & \hat{x} \leftarrow x_{k}-\left(x_{k}-x_{k-1}\right) \sqrt{\xi_{2}} \\
\text { otherwise } & \hat{x} \leftarrow x_{k-1}+\left(x_{k}-x_{k-1}\right) \sqrt{\xi_{2}}
\end{array}
$$


Examples - Sampling from Exponential PDF

$$
\begin{aligned}
& f(x)=\lambda e^{-x / \lambda}, \quad 0 \leq x \leq \infty \\
& F(x)=\int_{0}^{x} f\left(x^{\prime}\right) d x^{\prime}=1-e^{-x / \lambda}
\end{aligned}
$$

Direct Sampling:

$$
\text { solving } \xi=1-e^{-\hat{x} / \lambda} \quad \text { gives: } \hat{x} \leftarrow-\lambda \cdot \ln (1-\xi)
$$

$$
\begin{aligned}
& \text { Although }(1-\xi) \neq \xi \text {, } \\
& \text { both } \xi \text { and }(1-\xi) \text { are uniformly distributed on }(0,1) \text {, } \\
& \text { so we can use either in the random sampling procedure } \\
& \text { (i.e., the numbers are different, the distributions are the same) }
\end{aligned}
$$

$$
\hat{\mathbf{x}} \leftarrow-\lambda \cdot \ln \xi
$$

\section{Example - 2D Isotropic}

\section{Rejection (old vim)}

$$
f(\grave{\rho})=\frac{1}{2 \pi}, \quad \vec{\rho}=(u, v)
$$

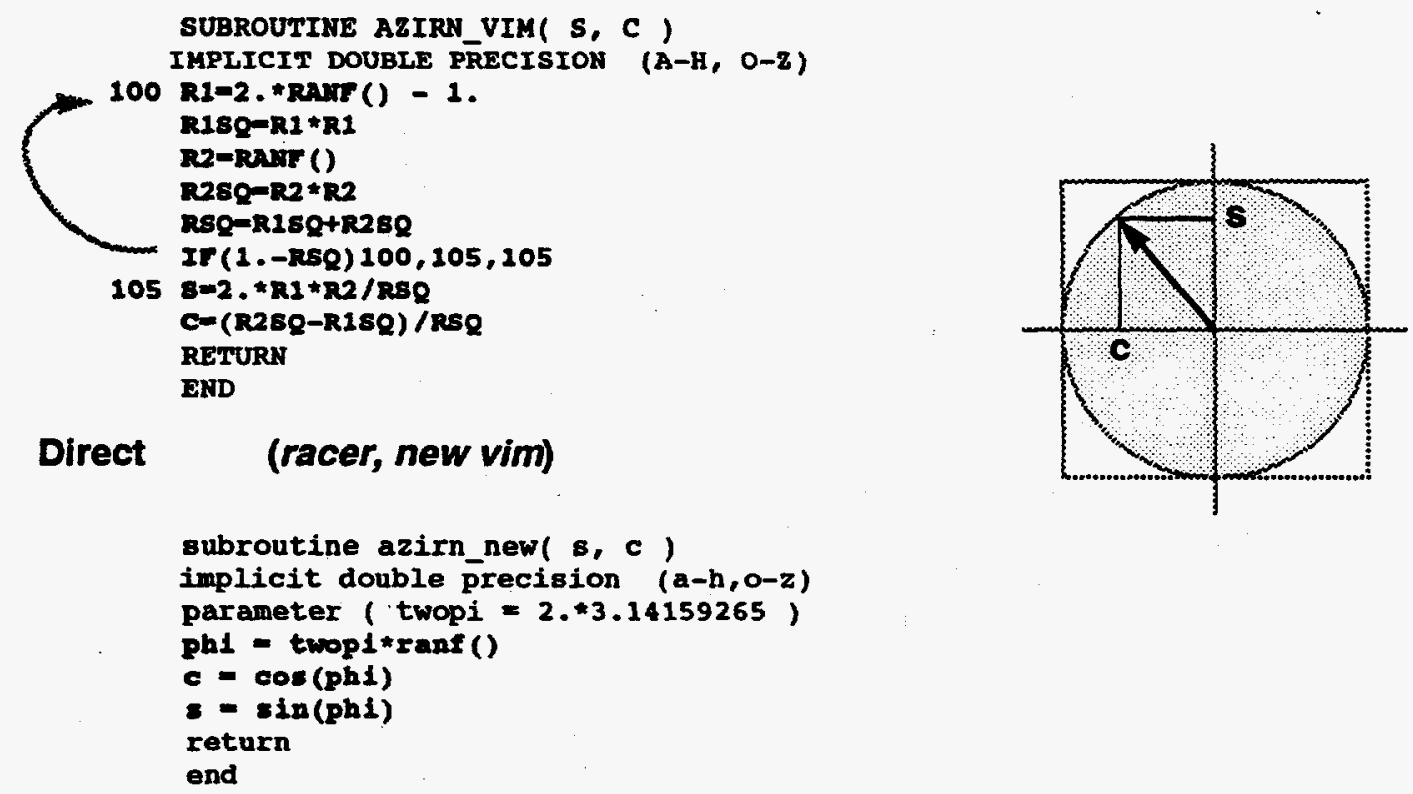




\section{Example - Watt Spectrum}

$$
f(x)=\frac{2 e^{-a b / 4}}{\sqrt{\pi a^{3} b}} e^{-x / a} \sinh \sqrt{b x}, \quad 0<x
$$

\section{Rejection (menp)}

- Based on Algorithm R12 from 3rd Monte Carlo Sampler, Everett \& Cashwell

- Define $K=1+a b / 8, L=a\left\{K+\left(K^{2}-1\right)_{1 / 2}\right\}, M=L / a-1$

- Set $\quad x \leftarrow-\log \xi_{1}, \quad y \leftarrow-\log \xi_{2}$

- If $\{y-M(x+1)\}^{2} \leq b L x, \quad$ accept: return $(L x)$ otherwise, reject

\section{Direct (new vim)}

- Sample from Maxwellian in C-of-M, transform to lab

$$
\begin{aligned}
& w-a\left(-\log \xi_{1}-\log \xi_{2} \cos ^{2} \frac{\pi}{2} \xi_{3}\right) \\
& x-w+\frac{a^{2} b}{4}+\left(2 \xi_{4}-1\right) \sqrt{a^{2} b w} \quad \begin{array}{r}
\text { (assume isotropic emission from fission fragment } \\
\text { moving with constant velocity in C-of-M) }
\end{array}
\end{aligned}
$$

- Unpublished sampling scheme, based on original Watt spectrum derivation

\section{Example - Linear PDF}

$$
f(x)=2 x, \quad 0 \leq x \leq 1
$$

\section{Rejection}

$$
\text { (strictly - this is not "rejection", but has the same flavor) }
$$

$$
\text { if } \begin{array}{rlr}
\xi_{1} \geq \xi_{2}, & \text { then } & \hat{x} \leftarrow \xi_{1} \\
& \text { else } & \hat{x} \leftarrow \xi_{2}
\end{array}
$$

or

$$
\hat{\mathbf{x}} \leftarrow \max \left(\xi_{1}, \xi_{2}\right)
$$

or

$$
\hat{x} \leftarrow\left|\xi_{1}-\xi_{2}\right|
$$

Direct

$$
\begin{aligned}
& F(x)=x^{2}, \quad 0 \leq x \leq 1 \\
& \hat{x} \leftarrow \sqrt{\xi}
\end{aligned}
$$




\begin{tabular}{|c|c|c|}
\hline \multicolumn{2}{|r|}{ Probability Density Function } & Direct Sampling Method \\
\hline Linear: & $f(x)=2 x$ & $x \leftarrow \sqrt{\xi}$ \\
\hline Exponential: & $f(x)=e^{-x}$ & $x \leftarrow-\log \xi$ \\
\hline 2D lsotropic: & $f(\vec{\rho})=\frac{1}{2 \pi}, \quad \vec{p}=(u, v)$ & $\begin{array}{l}u \leftarrow \cos 2 \pi \xi_{1} \\
v \leftarrow \sin 2 \pi \xi_{1}\end{array}$ \\
\hline 3D Isotropic: & $f(\vec{\Omega})=\frac{1}{4 \pi}$ & $\begin{array}{l}u \leftarrow 2 \xi_{1}-1 \\
v \leftarrow \sqrt{1-u^{2}} \cos 2 \pi \xi_{2} \\
w \leftarrow \sqrt{1-u^{2}} \sin 2 \pi \xi_{2}\end{array}$ \\
\hline Maxwellian: & $f(x)=\frac{2}{T \sqrt{\pi}} \sqrt{\frac{x}{T}} e^{-x / T}, \quad 0<x$ & $x \leftarrow T\left(-\log \xi_{1}-\log \xi_{2} \cos ^{2} \frac{\pi}{2} \xi_{3}\right)$ \\
\hline $\begin{array}{l}\text { Watt } \\
\text { Spectrum: }\end{array}$ & $f(x)=\frac{2 e^{-a b / 4}}{\sqrt{\pi a^{3} b}} e^{-x / a} \sinh \sqrt{b x}, \quad 0<x$ & $\begin{array}{l}w \leftarrow a\left(-\log \xi_{1}-\log \xi_{2} \cos ^{2} \frac{\pi}{2} \xi_{3}\right) \\
x \leftarrow w+\frac{a^{2} b}{4}+\left(2 \xi_{4}-1\right) \sqrt{a^{2} b w}\end{array}$ \\
\hline Normal: & $f(x)=\frac{1}{\sigma \sqrt{2 \pi}} e^{-\frac{1}{2}\left(\frac{x-\mu}{\sigma}\right)^{2}}$ & $x \leftarrow \mu+\sigma \sqrt{-2 \log \xi_{1}} \cos 2 \pi \xi_{2}$ \\
\hline
\end{tabular}

Random Sampllng - Computer Implementation

\section{Machine Considerations}

\section{Vector Hardware}

- Since $\sim 1980$, direct methods have been recommended for vectorization \& high performance on cray, cyber-205, sx-3, cm-2, ....

$\rightarrow$ Vector concepts apply directly to pipelined RISC cpu's (e.g., rs6000, i860, Fujitsu $\mu-v p, \ldots$ )

\section{Math Libraries}

- Many routines in math libraries are now table-driven, hence very fast

$\rightarrow$ fast $\sin , \cos$, sqrt, $\log , \& \exp$ functions

\section{RISC + Compiler Technology}

-Pipelining, concurrent ops, simple instructions, register-to-register ops, 64-bit hardware, better instruction scheduling, .....

$\rightarrow$ fast arithmetic (even for double-precision)

$\rightarrow$ Today, the most expensive operations are

- load/store

- IF...GOTO... 


\section{Software Considerations}

"Rules of thumb" for M.C. algorithm design have changed

- Nevertake the square root of a random number

- Avoid using $37 n$, cas, log, exp, r.....

- Use IF...GOTO, to to avoid arithmetic

- Random nafíbers are cheap, arithmettcis-expensive

Direct sampling methods have advantages

- Clear, succinct coding - easier to verify \& maintain

- Cpu time is comparable to rejection

- Direct methods vectorize efficiently

Aandom Sampling - Stratified Sampling

If a specific number of samples, $M$, is needed from a single distribution:

- Naive approach - repeat the sampling procedure $M$ times

- Stratified sampling approach

- partition the sample space into $M$ disjoint regions of equal probability

- produce 1 sample from each region

- Stratified sampling considerations

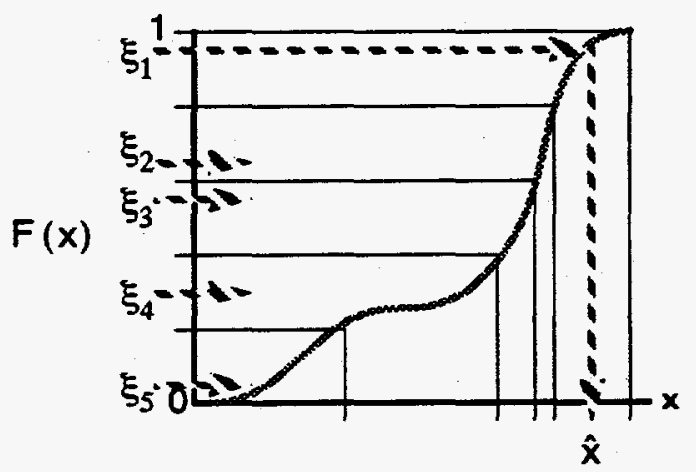

- $F(x)$ must be known \& easy to partition

- The number of partitions, $M$, must be known in advance

- Must be relatively easy to sample within each given partition

- Stratification improves the "coverage"

- Stratified sampling reduces variance, at little or no computing cost 
Rejection sampling methods are useful when it is difficult or

impossible to invert $F(x)$, or when $F(x)$ is not known

Example - Selection of initial source sites

- select a trial site:

$$
\begin{aligned}
& \hat{x} \leftarrow x_{1}+\left(x_{2}-x_{1}\right) \cdot \xi_{1} \\
& \hat{y} \leftarrow y_{1}+\left(y_{2}-y_{1}\right) \cdot \xi_{2}
\end{aligned}
$$

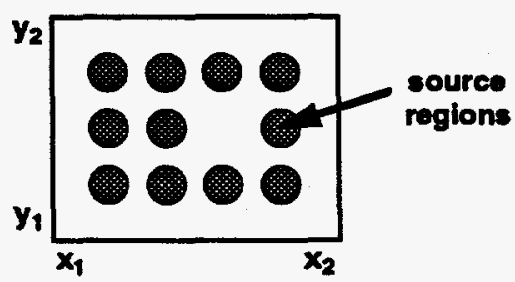

- if $(\hat{x}, \hat{y})$ is inside shaded region, then accept $(\hat{x}, \hat{y})$.

Otherwise, reject $(\hat{x}, \hat{y})$ and repeat.

Rejection methods are generally not used in KAPL Monte Carlo, since they are not well-suited to vectorization.

\section{Exceptions:}

- Selection of source sites

- every site, for neutrons from fixed-source

- sites for initial batch only, for neutrons from fission

- Delta-Tracking

Random Sampiling - Weighted Sampling

It is sometimes useful to sample from an alternate PDF

$$
f(x) d x=\left[\frac{f(x)}{g(x)}\right] g(x) d x=h(x) g(x) d x
$$

\& then "correct" the result via either weight factors or a 2nd sampling stage

\section{Welghted Sampling}

- To sample $\hat{x}$ from $f(x)$,

- first, sample $\hat{x}$ from $g(x)$

- then, multiply the "weight" assigned to $\hat{x}$ by $\frac{\text { right answer }}{\text { wrong answer }}=\frac{f(\hat{x})}{g(\hat{x})}$

- Note that $g(x)$ must be $>0$ whenever $f(x)>0$.

- Also, $g(x)$ must be normalized so that $\int g(x) d x=1$

\footnotetext{
- Example - survival blesing of collisions

If a collision occurs, $\quad P_{\text {survive }}=\frac{\Sigma_{S}}{\Sigma_{T}}$ is the probability of surviving.

Instead of sampling the outcome, always choose survival \& multiply the "weight" by $P_{\text {survive }}$
} 
- L. Devroye

Non-Uniform Random Variate Generation,

Springer-Verlag, New York (1986)

- C. J. Everett \& E. D. Cashwell

Third Monte Carlo Sampler, LA-9721-MS, Los Alamos National Lab. (1983)

- D. E. Knuth

The Art of Computer Programming, Volume 2 -

Seminumerical Algorithms, Addison-Wesley $\left(2^{\text {nd }}\right.$ ed., 1981)

- H. Kahn

Applications of Monte Carlo, AECU-3259,

Rand Corporation (1956)

- J. von Neumann

Various Techniques Used in Connection with Random Digits, NBS Applied Mathematics Series 12 (1951)

- G. Marsaglia \& T. A. Bray

A Convenient Method for Generating Normal Variables,

SIAM Review, 6 (1964)

Woighted Sampling Example - Effective Free-gas Model for Scatter with Bound Hydrogen

- Given a neutron with initial energy $E_{0}, E_{0}>.625 \mathrm{eV}$

- For scattering with free hydrogen (target-at-rest), PDF for scatter to $E$ is

$$
f_{F R E E}\left(E_{0} \rightarrow E\right)=\frac{1}{E_{0}}, \quad 0 \leq E \leq E_{0}
$$

- For scattering with bound hydrogen (free-gas), PDF for scatter to $E$ is

$$
\begin{array}{lll}
\text { down-scatter: } & f_{\text {BOUND }}\left(E_{0} \rightarrow E\right)=\frac{\text { erf } \sqrt{E / k T}}{E_{0}-k T / 2}, & 0 \leq E \leq E_{0} \\
\text { up-scatter: } & f_{\text {BOUND }}\left(E_{0} \rightarrow E\right)=\delta\left(E-E_{0}\right), & E>E_{0} \\
P(\text { upscatter })=\frac{k T}{E_{0}+k T / 2} &
\end{array}
$$

- Sampling scheme for $\hat{\mathrm{E}}, \hat{\mu}$ :

First, sample $\hat{E}, \hat{\mu}$ using target-at-rest scattering model.

Then,

$$
\text { If } \xi<P \text { (upscatter), } \quad \text { set } \hat{E} \leftarrow E_{0}, \hat{\mu} \leftarrow 1 \text {, then exit }
$$

Otherwise, modify weight by factor

$$
\begin{aligned}
& \frac{f_{\text {BOUND }}\left(E_{0} \rightarrow \hat{E}\right)}{f_{\text {FREE }}\left(E_{0} \rightarrow \hat{E}\right)}=\frac{\operatorname{erf} \sqrt{\hat{E} / k T}}{1-k T /\left(2 E_{0}\right)} \\
& \text { and set } \hat{\mu}-A_{\text {BOUND }}\left(E_{0} \rightarrow \hat{E}\right)
\end{aligned}
$$




\section{Sampling the free-flight distance, 8}

- To simulate the free-flight of particles through the problem geometry, need to randomly sample the distance to collision

- PDF for free-flight distance s, along current direction:

$$
f(s)=\frac{1}{\Sigma_{T}(s)} \exp \left(-\int_{0}^{s} \Sigma_{T}(x) d x\right)
$$

- If $\Sigma_{T}(x)$ is constant within a region, the PDF for that region simplifies to $f(s)=\frac{1}{\Sigma_{T}} \exp \left(-\Sigma_{T} \cdot s\right)$

- Sampling procedure is then: $\quad \hat{s} \leftarrow-\frac{1}{\Sigma_{T}} \ln \xi$

- For multiple regions, can stop at each boundary \& redetermine $\hat{\mathbf{s}}$.

Why is this OK?

Why is this OK?
- Note that probability of traversing distance $2 s=1-\int_{0}^{s} \frac{1}{\Sigma} e^{-\Sigma x} d x=e^{-\Sigma s}$
regions, note that

$$
e^{-\Sigma x_{1}} \cdot e^{-\Sigma x_{2}}=e^{-\Sigma\left(x_{1}+x_{2}\right)}=\text { probability of traversing both regions }
$$

\section{"Regular" Tracking}

- Move particles through one region at a time, until collision occurs

- Can be expensive if many regions must be traversed before collision

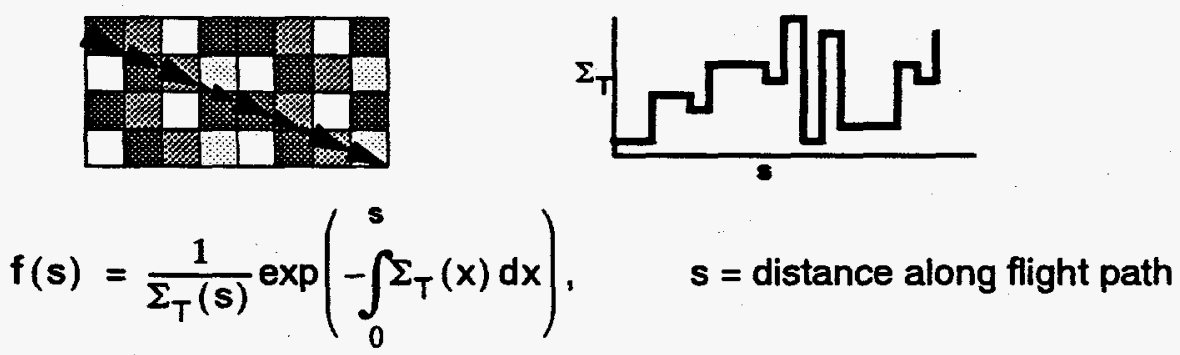

- "Regular" tracking procedure, when $\Sigma_{\mathrm{T}}$ constant within each region:

- Sample a flight distance $\hat{s}$ using $\Sigma_{T}$ for current region: $\hat{s} \leftarrow-\frac{1}{\Sigma_{T}} \ln \xi$

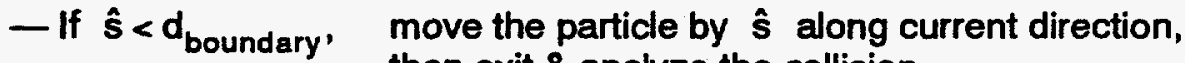
then exit \& analyze the collision

- Otherwise, move the particle by $d_{\text {boundary }}$ along current direction, repeat until collision 


\section{Delta Tracking}

- A type of rejection method for sampling the free-flight distance

- Also called Woodcock tracking, fast tracking, or hole tracking

- Useful when $\Sigma_{T}$ varies rapidly over the ffight path

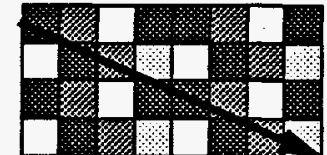

$$
f(s)=\frac{1}{\Sigma^{*}} \exp \left(-\Sigma^{*} \cdot s\right) \text {, }
$$

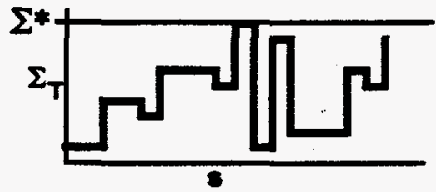

s = distance along flight path

- For delta tracking, a fictitious cross-section $\Sigma^{*}$ is used, rather than $\Sigma_{T}(s)$

- $\Sigma^{*}$ should be chosen to be $\geq \Sigma_{T}$ (s) for all possible points along path

$-\Sigma^{*}$ may be a function of particle energy, or not

$-\Sigma^{*}=\Sigma_{T}(s)+\Sigma_{\delta}(s)=$ constant, $\quad \Sigma_{\delta}(s) \geq 0$ for all $s>0$

where $\Sigma_{\delta}(s)=$ cross-section for "delta-scattering", i.e., scatter with no change in energy or direction, a fictitious scattering event, or "pseudo-collision"

Delta Tracking procedure:

- $\Sigma^{*}=\Sigma_{T}(s)+\Sigma_{\delta}(s)=$ constant

- $\Sigma^{*} \geq \Sigma_{\mathrm{T}}(\mathrm{s})$ and $\Sigma_{\delta}(s) \geq 0$ for all $s>0$

- To sample the distance to collision, $\hat{\mathbf{s}}$, using delta tracking, play the following rejection game:

- set $\hat{s} \leftarrow 0$

- repeat the following steps until a (real) collision occurs:

- sample a flight distance $\hat{x}$ using $\Sigma^{*}: \quad \hat{x} \leftarrow-\frac{1}{\Sigma^{*}} \ln \xi$

$\cdot \hat{s} \leftarrow \hat{s}+\hat{x}$

- with probability $\frac{\Sigma_{\delta}(\hat{\mathbf{s}})}{\Sigma^{*}}, \quad$ reject the collision site \& repeat. otherwise, accept $\hat{s}$ \& analyze the collision 
Delta tracking can be effective if at least some of the following are true:

- The cost of locating a particle position in the problem geometry is small, relative to the cost of computing many distances

- $\Sigma *$ is not too different from the "average" $\Sigma_{T}(s)$

- $1 / \Sigma^{*}$ is large compared to distances between geometric boundaries

$\rightarrow$ All of these considerations are (usually) true for fast neutrons in grid regions

Delta tracking can be ineffective if a few small regions have very large $\Sigma_{T}$

- Results in $\Sigma^{*} » \Sigma_{T}(s)$ for most regions, so that efficiency of rejection sampling is low

If the cross-sections are continuously-varying functions of position, then delta-tracking may be the only feasible method for sampling s

- Same delta-tracking procedure is used if cross-sections vary continuously with position \&/or energy.

- Can even be used when the cross-sections are not known in advance, so long as the maximum cross-section can be determined.

Random Sampling - Dolta Tracking

[continued]

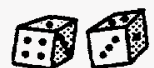

Proof: - Delta-Tracking is an unbiased method for sampling the free-flight distance

Consider the probability of traversing a distance $s$ along the flight path without undergoing a (real) collision, $P(s)$.

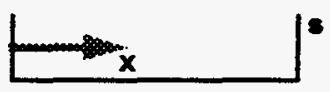

- $\Sigma^{*}=\Sigma_{T}(s)+\Sigma_{\delta}(s)=$ constant, $\quad \Sigma^{*} \geq \Sigma_{T}(s)$ and $\Sigma_{\delta}(s) \geq 0$ for all $s>0$

- For convenience, define optical thicknesses for real scatter \& delta scatter:

$$
\tau(s)=\int_{0}^{s} \Sigma_{T}(x) d x \quad \tau_{\delta}(s)=\int_{0}^{s} \Sigma_{\delta}(x) d x
$$

Note that, by definition, $\quad \Sigma * s=\tau(s)+\tau_{\delta}(s) \quad$ and $\quad \Sigma * s \geq \tau(s)$

- For a particular flight, there could be exactly $0,1,2, \ldots . ., \infty$ pseudo-collisions before a real collision occurs. 
- Let $P(s \mid n)=$ probability of traversing distance $s$ along the flight path with exactly $\mathbf{n}$ pseudo-collisions

$$
\begin{aligned}
& \text { Then, } P(s)=\sum_{n=0}^{\infty} P(s \mid n) \\
& P(s \mid 0)=e^{-\Sigma * s} \\
& s \quad s \\
& P(s \mid 1)=\int_{0} P(x \mid 0) \Sigma_{\delta}(x) P(s-x \mid 0) d x=\int_{0} e^{-\Sigma * x} \Sigma_{\delta}(x) e^{-\Sigma *[s-x]} d x=\tau_{\delta}(s) e^{-\Sigma * s} \\
& \text { s s } \\
& P(s \mid 2)=\int_{0}^{s} P(x \mid 1) \Sigma_{\delta}(x) P(s-x \mid 0) d x=\int_{0} \tau_{\delta}(x) e^{-\Sigma^{*} x} \Sigma_{\delta}(x) e^{-\Sigma^{*}[s-x]} d x \\
& =\int_{0}^{s} \tau_{\delta}(x) \Sigma_{\delta}(x) e^{-\Sigma * s} d x=\frac{\left[\tau_{\delta}(s)\right]^{2}}{2} e^{-\Sigma * s} \\
& P(s \mid n)=\int_{0}^{s} P(x \mid n-1) \Sigma_{\delta}(x) P(s-x \mid 0) d x=\frac{\left[\tau_{\delta}(s)\right]^{n}}{n !} e^{-\Sigma^{*} s}
\end{aligned}
$$

- Then, the total probability of traversing a distance $s$ without undergoing a (real) collision is

$$
\begin{aligned}
P(s) & =\sum_{n=0}^{\infty} P(s \mid n)=\sum_{n=0}^{\infty} \frac{\left[\tau_{\delta}(s)\right]^{n}}{n !} e^{-\Sigma^{*} s}=e^{\tau_{\delta}(s)} e^{-\Sigma^{*} s} \\
& =\exp \left(\tau_{\delta}(s)-\Sigma^{*} s\right)=\exp [-\tau(s)]=\exp \left(-\int_{0}^{s} \Sigma_{T}(x) d x\right)
\end{aligned}
$$

$\rightarrow$ This is the correct result, identical to the "Regular Tracking" procedure. 


\section{Combined Russian Rouletting \& Splitting}

- Russian Roulette - kill off some particles, but conserve total weight

- to save computing time, roulette "unimportant" particles

- Splitting

- create extra identical particles, but conserve total weight

- to reduce variance, split particles if weights "too large"

- Definitions

wgt = Particle weight

For the region containing the particle:

$\mathbf{w}_{\text {high }}=$ upper bound on weight, if wgt larger - split

$w_{\text {low }}=$ lower bound on weight, if wgt lower - roulette

Then,

$w_{\text {ave }}=$ weight to assign survivors, $w_{\text {low }}<w_{\text {ave }}<w_{\text {high }}$

wgt $/ w_{\text {ave }}=$ probability of surviving split/roulette

For each region, choose $w_{\text {ave }}$ based on region "importance" (using adjoint function, if known). Choose $w_{\text {low }} \& w_{\text {high }} 2-5$ times lower or higher.

- Combined game for split/roulette:

$$
\begin{aligned}
& \text { if } \mathbf{w g t}<w_{\text {low }} \text { or } w_{\text {high }}<w g t, \\
& \text { create } n \text { particles of weight } w_{\text {ave, }} \text { where } n \leftarrow\left\lfloor\frac{w g t}{w_{\text {ave }}}+\xi\right\rfloor
\end{aligned}
$$

\section{Random Sampling in RACER}

\section{- Source}

- Fixed sites - uniform PDF + rejection

- Fission sites - discrete PDF + stratified sampling

- Energy - piecewise-linear PDF (binary table search + linear PDF)

- Direction - isotropic 3D PDF

- Tracking

- free-flight distance - exponential PDF

- delta-tracking - rejection sampling of pseudo- \& real collisions

- Russian Roulette \& Splitting

- discrete PDF + weights

- Collisions

- Survival biasing - weights

- Select phase \& nuclide - discrete PDF (on-the-fiy)

- Epithermal

- Scattering angle - equally-probable histograms (uniform discrete PDF + uniform in bin)

- Inelastic: energy - discrete PDF (aliased), then uniform within group

modified freo-gas

- Thermal n2n - weights

- multigroup - discrete PDF for group-to-group (aliased), linear PDF for $\mu$

- $S(\alpha, \beta)$ - discrete PDF (aliased) \& uniform PDF sampling

- Direction - polar angle from uniform PDF

- Fission bank - discrete PDF + weights 


\section{Computational}

\section{Geometry}

Engineering Model vs. Computational Model
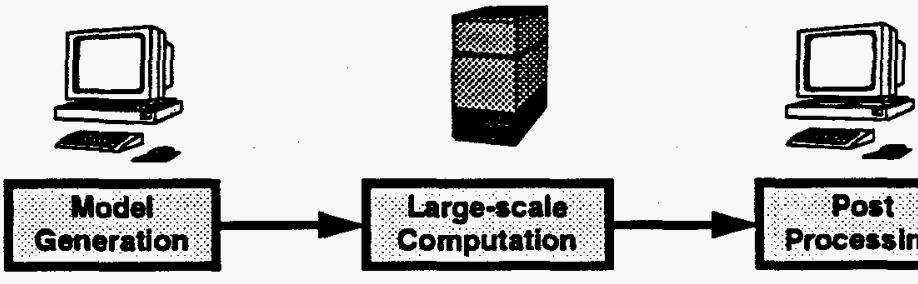

engineering model

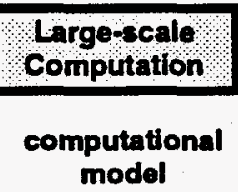

\section{Processing}

engineering

model

- Model Generation

- focus on engineering productivity

- describes "reality" to computer

- interactive, batch, or CAD

- Large-scale Computation

- focus on efficiency \& capabilities

- data structure should be compact \& regular

- computational model often hidden from user

- best reference: source coding

- Post-Processing

- interpretation of results

- visualization 
- Element geometry

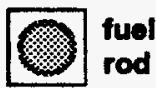

rod

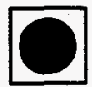

control rod

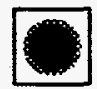

bumable

absorber

- Elements $\rightarrow$ Assemblies

- Assemblies $\rightarrow$ Core

- Core + peripherals $\rightarrow$ 3-D Model
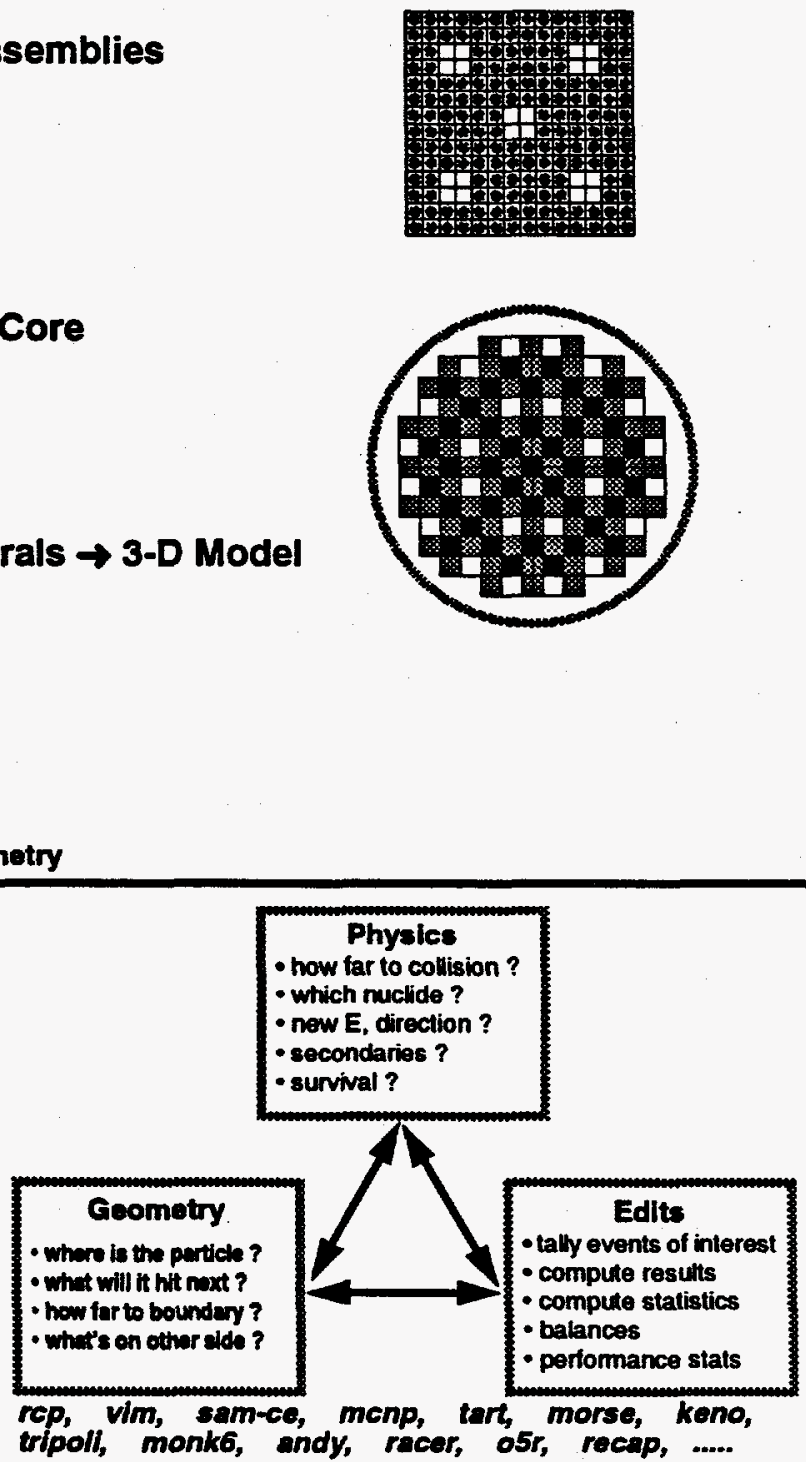

Development of particular capabilities is driven by applications:

- Shielding \& experiment analysis

$$
\begin{aligned}
& \text { - irregular geometry } \\
& \text { - moderate number of regions } \\
& \text { - few compositions } \\
& \Rightarrow \text { focus: convenient, flexible input for arbitrary geometry }
\end{aligned}
$$

- Reactor core analysis

$$
\begin{aligned}
& \text { - regular geometry } \\
& \text { - many regions (Up to } \sim 10^{8} \text { ) } \\
& \text { - many compositions (Up to } \left.\sim 10^{5}\right) \\
& \Rightarrow \text { focus: automated processing for repeating, detailed geometry }
\end{aligned}
$$




\section{RACER Computational Algorithm - Geometric View}

repeat for all batches

- repeat for all cross-section supergroups

. repeat until neutrons are gone

. . repeat until collision

. . . repeat for surfaces of 3-D region

. . . . distance calculation

. $. \quad . \quad . \quad \ldots$

. . . . repeat while in grid

. . . . . d dstance calculation ta

. . . . . repeat for figures in grid region

. . . . . . . repeat for quadrics in figure

- distance calculation

1 reactor calculation

$$
\Downarrow
$$

$\sim 10^{\circ}$ distance calculations

\section{RACER Computational Events}

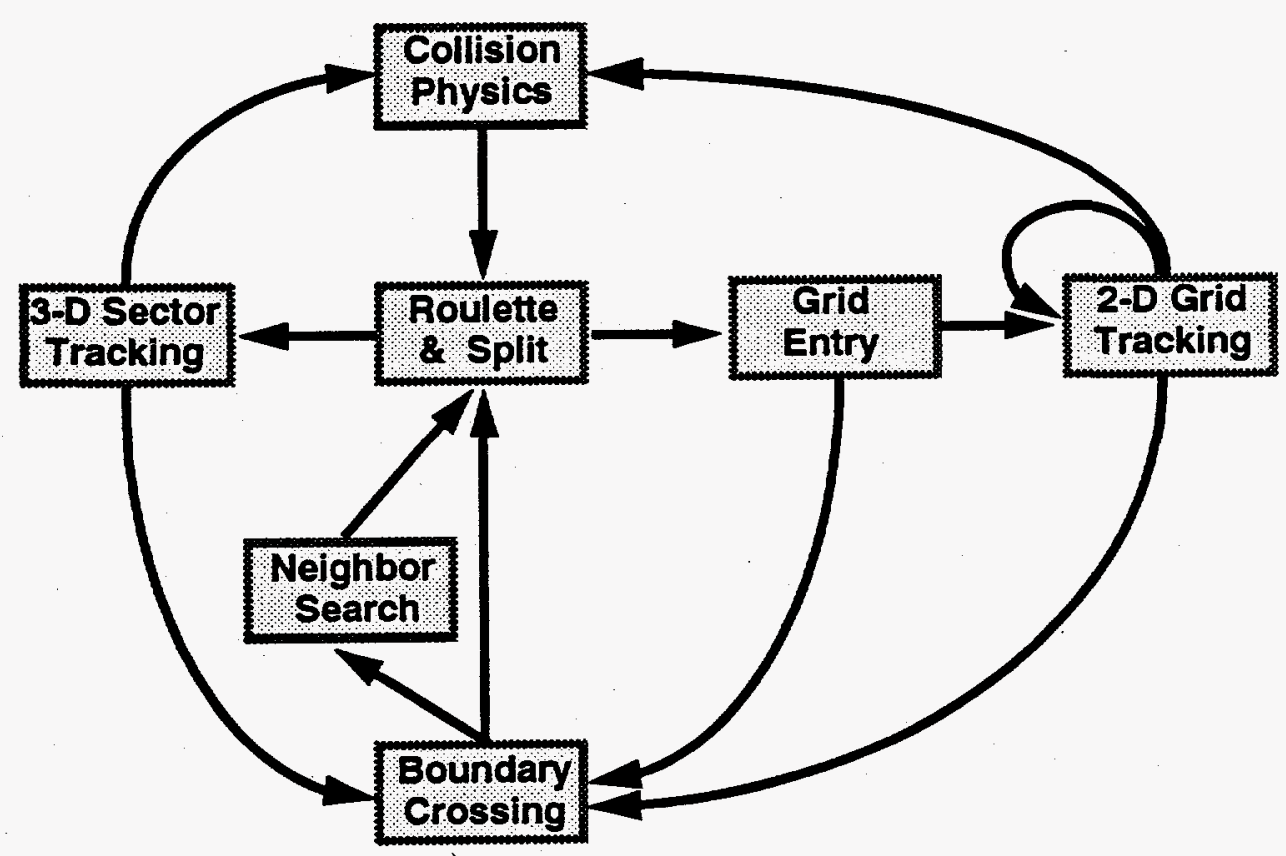


RACER - General Geometry Capabilities

\section{3-D general geometry}

- allowed surface types:

general planes, sphere, right circular cylinder, right elliptic cylinder, ellipsoid, skewed cylinders, general 3-D quadratic

- 3-D regions (sectors) defined by lists of surfaces (with sense \& BC)

- Sectors may be combined into edit-media

- Neighbor lists built dynamically

\section{Sectors may have embedded detailed grids}

- lattice (need not be rectangular)

- general rotation/translation/scaling to/from 3-D geometry

- lattice "boxes" may contain arbitrary sets of quadratics

- detailed lattice regions may be assigned to arbitrary edit-media

Delta-tracking or surface-to-surface tracking, variable by energy

- Delta-tracking - also called "Woodcock tracking" or "hole geometry"

large problem example: $>50003-D$ sectors, $>10^{7}$ detailed regions

\section{RACER 3D Geometry}

\section{Surface}

- Linear or quadratic polynomial, in absolute coordinates $(x, y, z)$

$$
F(x, y, z)=a x^{2}+b y^{2}+c z^{2}+d x y+e y z+f z x+g x+h y+i z+j
$$

- Normalization is arbitrary

- RACER Convention: factor of leading $2^{\text {nd }}$ order term is positive, usually $=1.0$

- Surface is defined by: $\quad F(x, y, z)=0$

- Surfaces are infinite in extent

- Sense:

- relationship between a point in space $\left(x_{0}, y_{0}, z_{0}\right)$ \& a surface $F(x, y, z)$

$$
\begin{array}{ccc}
\text { - point }\left(x_{0}, y_{0}, z_{0}\right) & \text { is inside } & \text { if } F\left(x_{0}, y_{0}, z_{0}\right)<0 \\
\text { outside } & \text { if } F\left(x_{0}, y_{0}, z_{0}\right)>0 \\
\text { on } & \text { if } F\left(x_{0}, y_{0}, z_{0}\right)=0
\end{array}
$$




\section{RACER Surface Equations}

\begin{tabular}{|c|c|c|}
\hline Туре & Description & Equation: $F(x, y, z)=0$ \\
\hline 1 & general plane & $a x+b y+c z+d=0$ \\
\hline 2 & right cylinder & $a(x+b)^{2}+(y+c)^{2}+d=0$ \\
\hline 3 & cylinder skewed in Z & $a(x+b+c z)^{2}+(y+d+e z)^{2}+f=0$ \\
\hline 4 & sphere & $(x+a)^{2}+(y+b)^{2}+(z+c)^{2}+d=0$ \\
\hline 5 & ellipsoid & $a(x+b)^{2}+c(y+d)^{2}+e(z+f)^{2}+g=0$ \\
\hline 6 & general 3-D quadric & $a x^{2}+b y^{2}+c z^{2}+d x y+e y z+f z x+g x+h y+i z+j=0$ \\
\hline 7 & pair of z-independent planes & $(x+b y+c)^{2}-d^{2}=0$ \\
\hline 11 & $x=$ constant plane & $x+d=0$ \\
\hline 12 & $y=$ constant plane & $y+d=0$ \\
\hline 13 & $z=$ constant plane & $z+d=0$ \\
\hline 14 & z-independent plane & $x+b y+d=0$ \\
\hline 21 & pair of planes parallel to $Y Z$ & $(x+c)^{2}-d^{2}=0$ \\
\hline 22 & pair of planes parallel to $X Z$ & $(y+c)^{2}-d^{2}=0$ \\
\hline
\end{tabular}

\section{RACER 3D Geometry}

Side

- half-space, defined by signed surface number

$$
\text { e.g., } \quad+7 \rightarrow\left\{(x, y, z) \mid F_{7}(x, y, z)>0\right\}
$$

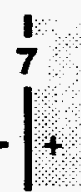

\section{Sector}

- 3-D region, intersection of sides

- note: sectors are defined only by the intersection of sides; unions of sides are not allowed

- defined by list of sides

e.g., Sector $3==-1,+4,+7,-523,-734$

- Sense:

- relationship between a point in space $\left(x_{0}, y_{0}, z_{0}\right) \&$ a sector

- point $\left(x_{0}, y_{0}, z_{0}\right)$ is inside if all surface-senses match, outside otherwise

- attributes: - edit medium number

- importance region number

- boundary conditions for each surface (R,E,P,N)

- embedded geometry (grid) - optional 
Sector Geometry - Examples

Example 1

Surface 1: sphere

Sector 1 :<smiles>[CH]CC[IH]CCC(C#C)C#C</smiles>

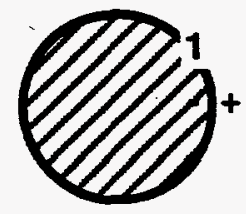

\section{Example 2}

Surface 1: sphere

Surface 2: plane

Sector 1: $-1,-2$

\section{Example 3}

\section{$\infty \infty$}

Surface 1: sphere

Surface 2: plane

Surface 3: plane

Sector $1:-1,-2$

Sector $2:-1,+2,+3$

Edit-medium 1: 1,2 $\quad X+\%$

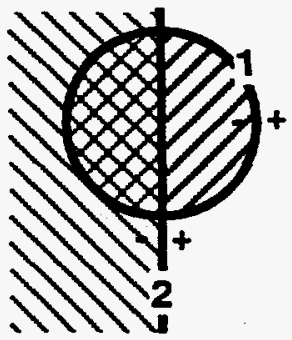

Computational Geometry

\section{Sector Geometry Example: $\quad$ Box with a Hole}

- Define surfaces:

planes: $1,2,3,4$

5,6

(sides)

(top, bot)

sphere: 7

(problem origin at lower rear)

- Define sectors

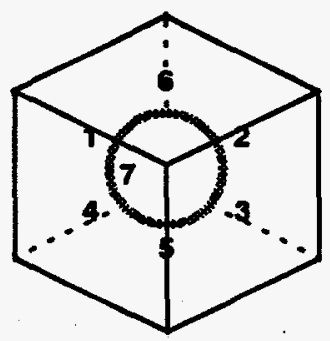<smiles>[Y]C([CH])C(C)C</smiles>

Sector 1: inside the sphere

$-7$

Sector 2: inside the box, but outside the sphere $+7,+1,+2,-3,-4,+5,-6$

- Assign properties to each sector

- define the boundary conditions for each surface of the sector

- assign an edit-medium number to the sector

- assign a composition (material) to the edit-medium

- assign an importance region number to the sector 
Sector Geometry - Miscellaneous

- Sectors do not have to be convex regions

Sector $1: \quad+1,-2,-3,+4,+5,+6$

Sector 2 : $\quad-5$

Sector 3 : $\quad-6$

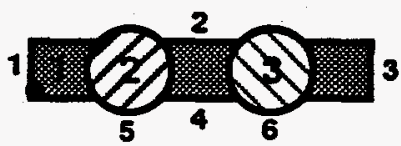

- Sectors may be infinite in extent

- For 2-D problems, sectors do not need a "top" \& "bottom"

- Adjoining sectors must share a common surface (with opposite senses)

- No gaps or overlap permitted

- "Similar" surfaces must be combined

- Sectors cannot be defined as unions of sides

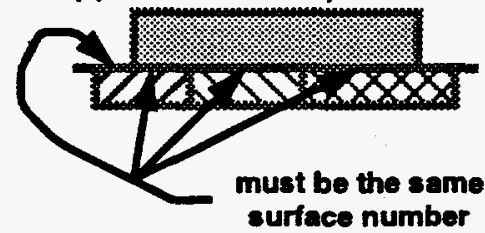

- Not a valid sector:

- Note: can do this by combining sectors into edit-media

Computatlonal Geometry

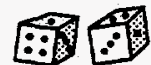

[96]

Sector Geometry - Lists, Logic, Lookups

Sector Data

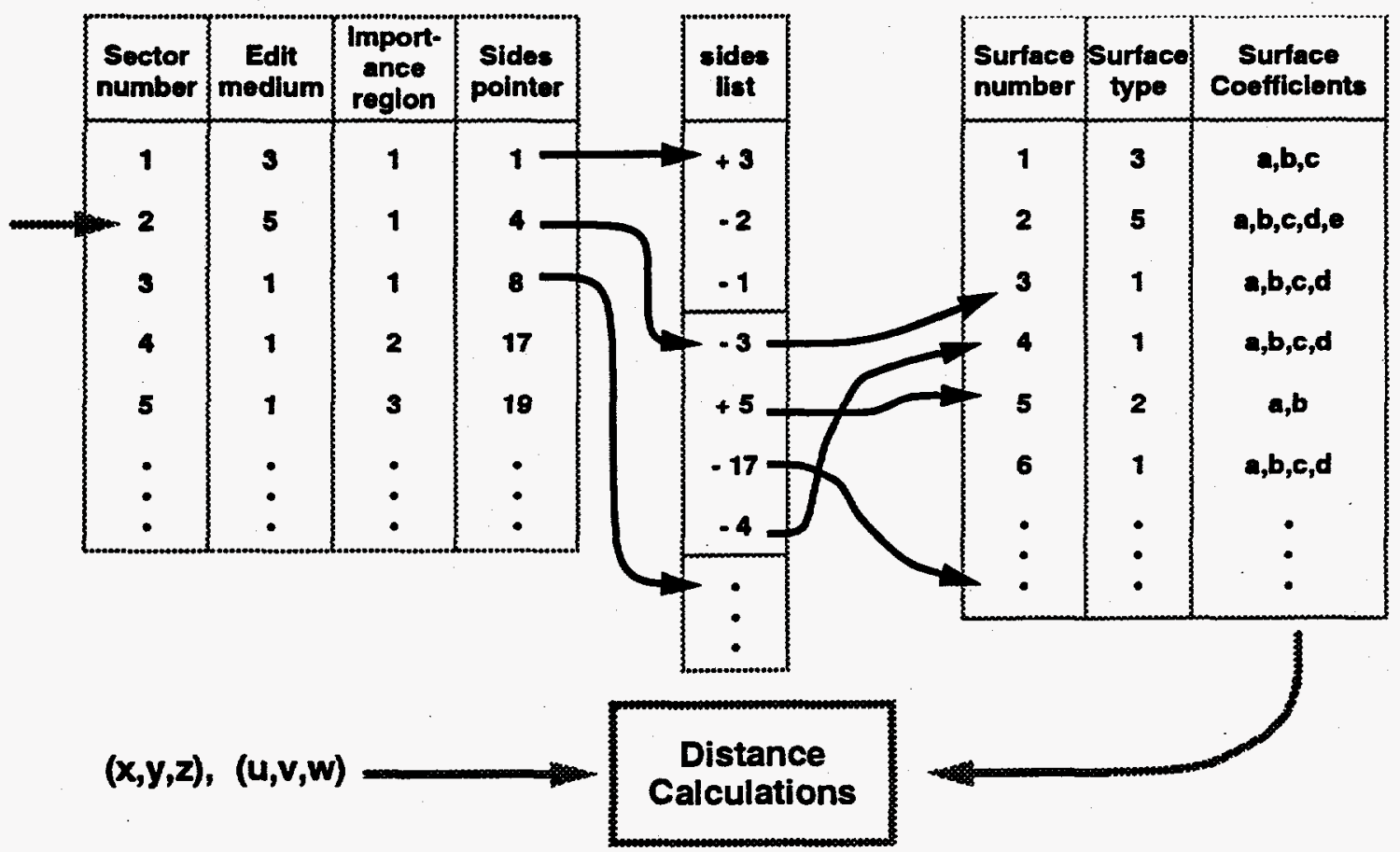


Distance Calculations in 3D Geometry

30 Surface: $\quad F(x, y, z)=0$

$\begin{array}{lll}\text { "linear" } & \rightarrow & \nabla F=\text { constant } \\ \text { "quadratic" } & \rightarrow & \nabla F=f(x, y, z), \quad \nabla^{2} F=\text { constant }\end{array}$

\section{Distance Calculation}

- 8 = directed distance from $\left(x_{0}, y_{0}, z_{0}\right)$ along $(u, v, w)$ to $F(x, y, z)$

= smallest positive root of $F\left(x_{0}+s u, y_{0}+s v, z_{0}+s w\right)=0$

- recast as

$$
G(0)+\int_{0}^{s} \frac{\partial G}{\partial s^{\prime}} d s^{\prime}=0, \quad \text { where } G(s)=F\left(x_{0}+s u, y_{0}+s v, z_{0}+s w\right)
$$

- then, $\left[\frac{1}{2} \frac{\partial^{2}}{\partial s^{2}} G(0)\right] s^{2}+\left[\frac{\partial}{\partial s} G(0)\right] s+[G(0)]=0$

- Or.

$$
A s^{2}+2 B s+C=0, \quad D=B^{2}-A C
$$

[continued]

\section{Distance Calculations in 3D Geometry}

- $A s^{2}+2 B s+C=0$,

$$
D=B^{2}-A C
$$

- 27 combinations of $A, B, C>0,<0,=0$

- only 12 yield valid solutions:

$$
\begin{aligned}
& s=-\frac{C}{2 B} \text { if }(A=0, C<0, B>0) \quad \text { or }(A=0, C>0, B<0) \\
& =\frac{-B-\sqrt{D}}{A} \text { if }(A>0, C>0, B<0, D>0) \text { or }(A<0, C>0, B>0, D>0) \\
& =\frac{-B+\sqrt{D}}{A} \text { if }(A>0, C<0, B>0, D>0) \text { or } \quad(A>0, C<0, B<0, D>0) \\
& \text { or }(A>0, C<0, B=0, D>0) \quad \text { or } \quad(A<0, C<0, B>0, D>0) \\
& \text { or }(A>0, C=0, B<0, D>0) \text { or }(A<0, C=0, B>0, D>0)
\end{aligned}
$$

- Use known surface-sense, f, to solve roundoff problems \& eliminate tolerance check

$$
\begin{aligned}
s^{\prime} & =-\frac{C}{2 B} \quad \text { if } \quad(A=0, D>0) \\
& =\frac{-B-\sqrt{D}}{A} \text { if }(A=0, D>0,5>0) \\
& =\frac{-B+\sqrt{D}}{A} \text { if }(A=0, D>0,5<0) \\
& =\infty \text { otherwise } \\
s & =s^{\prime} \text { if } s^{\prime}>0 \\
& =\infty \text { otherwise }
\end{aligned}
$$

- Distance to Sector Boundary = minimum s for all surfaces of sector 
Sector Tracking - outline of calculation

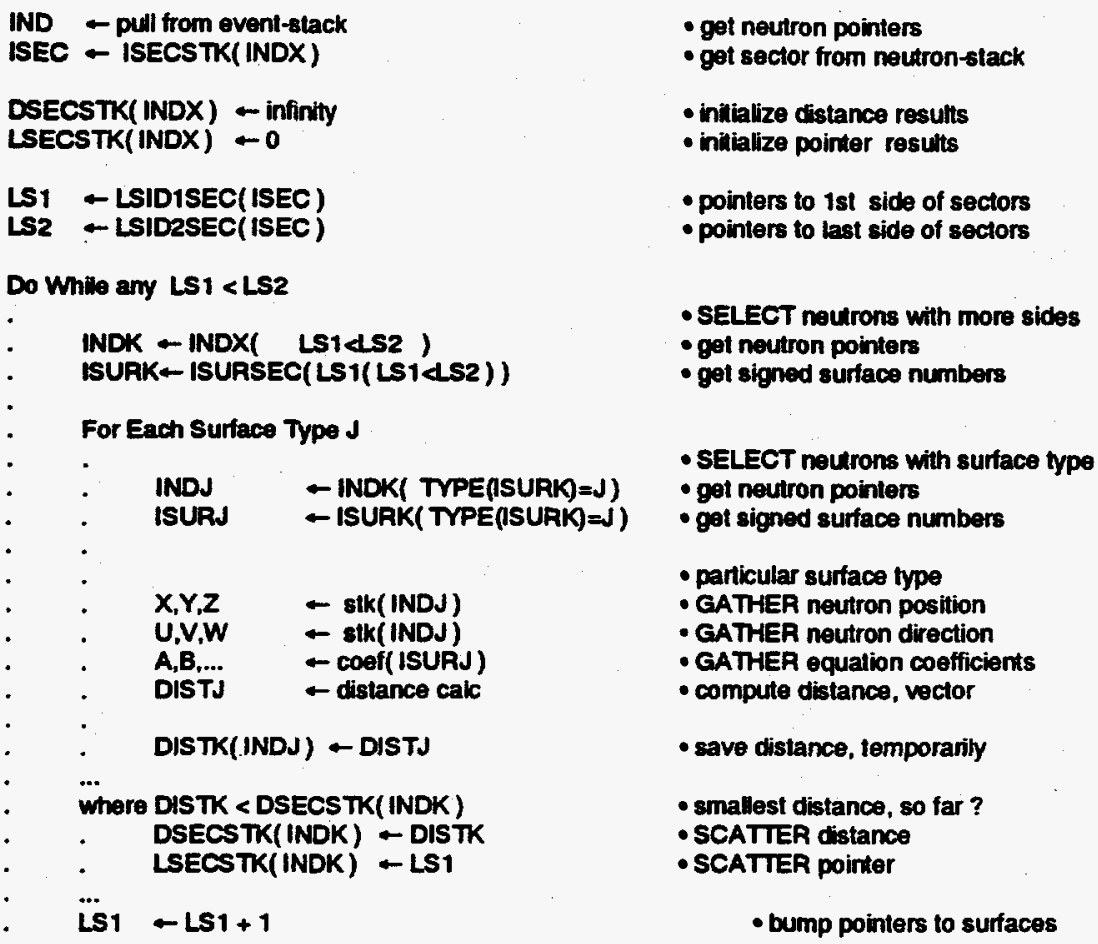

- bump pointers to surfaces

Computational Geometry

RACER Embedded Lattice Geometry

Grid: $\quad$ embedded within 3-D sector

- detailed 2-D geometry extruded, axially uniform

- 2-D mesh of parallel lines, need not be rectangular
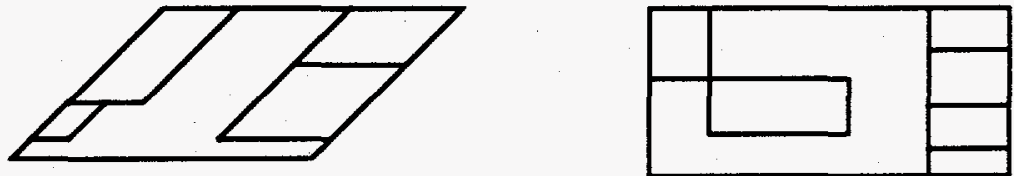

- grid lines are clipped at grid boundaries

- local coordinates - origin at lower left corner

- general transformation to/from 3-D: rotate, reflect, scale, translate

- grid box may contain composition or embedded figures

- very compact storage, much faster tracking

Figure: $\quad$ arbitrary types and numbers of 2-D quadrics

- constraint: quadrics do not intersect within grid box

- local coordinates

- translate toffrom grid coordinates

- clipped at grid box boundaries

Quadrics: - general 2nd order surfaces, $F(x, y)$

- local coordinates

- clipped at grid box boundaries 


\section{RACER Grid Geometry}

- Within a grid box, quadric surfaces can be nested. Inner surfaces overlay outer ones.

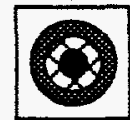

- Quadrics are clipped at grid box boundaries

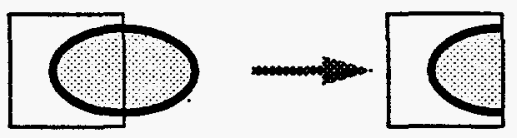

- Quadrics cannot intersect within a grid box. Extra grid lines must be inserted at intersections.

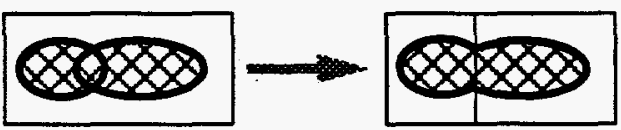

- Lines which are not parallel to the grid must be represented by infinite ellipses

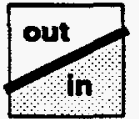

- A single figure can be repeated via translation to different grid boxes (no rotation)

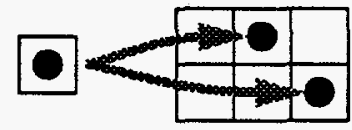

Computational Geometry

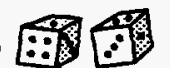

\section{Location \& Distances within Grids}

- Location - determine grid-box $(i, j)$

I: binary search to find $x$-interval containing $x$

j: binary search to find $y$-interval containing $y$

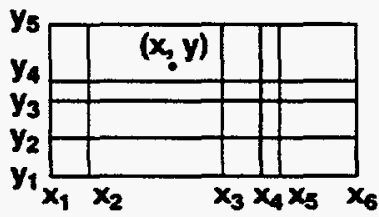

- Distance to boundary of grid-box

- use signs of $(u, v)$ to select the next $x$-bound \& $y$-bound:

$$
\begin{array}{lll}
\text { if } u<0, \quad \bar{x} \leftarrow x_{i}, & \text { otherwise } & \bar{x} \leftarrow x_{i+1} \\
\text { if } v<0, \quad \bar{y} \leftarrow y_{j}, & \text { otherwise } & \bar{y} \leftarrow y_{j+1}
\end{array}
$$

- compute $x$-distance \& $y$-distance

$$
d_{x}=\frac{\bar{x}-x}{u} \quad d_{y}=\frac{\bar{y}-y}{v}
$$

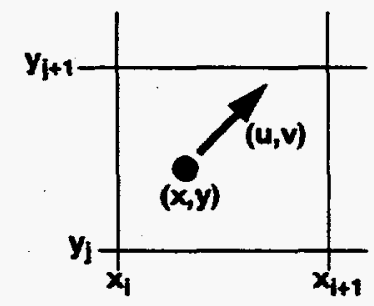

- if grid-box contains quadrics, compute q-distance, $d_{q}$

- distance to next grid-boundary $=d_{\text {grid }}=\min \left(d_{x}, d_{y}, d_{q}\right)$

$-d=\min \left(d_{\text {sector, }}, d_{\text {grid }}\right)$ 


\section{Grid Tracking}

Grid Entry: $\quad$ - transform $(x, y, z)$ and $(u, v, w)$ from 3-D to local - $(u, v, w)$ not normalized

$$
\left[\begin{array}{l}
x \\
y \\
z
\end{array}\right]_{\text {Grid }}=\left[\begin{array}{lll}
g_{1} & g_{2} & g_{3} \\
g_{5} & g_{6} & g_{7} \\
g_{9} & g_{10} & g_{11}
\end{array}\right]\left[\begin{array}{l}
x \\
y \\
z
\end{array}\right]_{\text {Sector }}+\left[\begin{array}{l}
g_{4} \\
g_{8} \\
g_{12}
\end{array}\right] \quad\left[\begin{array}{l}
u \\
v \\
w
\end{array}\right]_{\text {Grid }}=\left[\begin{array}{lll}
g_{1} & g_{2} & g_{3} \\
g_{5} & g_{6} & g_{7} \\
g_{9} & g_{10} & g_{11}
\end{array}\right]\left[\begin{array}{l}
u \\
v \\
w
\end{array}\right]_{\text {Sector }}
$$

Grid Locate: - binary searches of $x \& y$ grid-line intercepts

Grid Distance: - select $x \&$ y grid-lines using signs of $u \& v$

- simple distance calculation (if not using delta-tracking)

Figures: - for each figure, check all quadrics

Quadrics: $\quad$ translate $x \& y$

- compute distance (if not using delta-tracking) \& sense

Lookups: - edit-medium, composition

Grid Exit: $\quad$ transform back to 3-D, if collision or grid boundary cross

RACER Boundary Conditions - for Each Surface of Sector

Normal: - continue neutron flight, no action at boundary

Exit: $\quad$ leakage out of problem, tally \& kill

Reflect: $\quad$ Plane- $a x+b y+c z+d=0$, with $a^{2}+b^{2}+c^{2}=1$

$$
\left[\begin{array}{l}
u \\
v \\
w
\end{array}\right]_{\text {New }}=\left[\begin{array}{l}
u \\
v \\
w
\end{array}\right]_{\text {Old }}-2(a u+b v+c w) \cdot\left[\begin{array}{l}
a \\
b \\
c
\end{array}\right]
$$

- Cylinder- $a(x+b)^{2}+(y+c)^{2}+d=0$

$$
\left[\begin{array}{l}
u \\
v \\
w
\end{array}\right]_{\text {Now }}=\left[\begin{array}{l}
u \\
v \\
w
\end{array}\right]_{\text {Oid }}-2 \frac{(u x+v y)}{|d|} \cdot\left[\begin{array}{l}
x \\
y \\
0
\end{array}\right]
$$

Periodic: - rotate about point $\left(x_{p}, y_{p}, 0\right)$ by angle $\theta$, with $C_{p}=\cos \theta, S_{p}=\sin \theta$

$$
\left[\begin{array}{l}
x \\
y \\
z
\end{array}\right]_{\text {New }}=\left[\begin{array}{ccc}
c_{p} & -s_{p} & 0 \\
s_{p} & c_{p} & 0 \\
0 & 0 & 1
\end{array}\right]\left[\begin{array}{c}
x-x_{p} \\
y-y_{p} \\
z
\end{array}\right]_{\text {Old }}+\left[\begin{array}{l}
x_{p} \\
y_{p} \\
0
\end{array}\right] \quad\left[\begin{array}{l}
u \\
v \\
w
\end{array}\right]_{\text {New }}=\left[\begin{array}{ccc}
c_{p} & -s_{p} & 0 \\
s_{p} & c_{p} & 0 \\
0 & 0 & 1
\end{array}\right]\left[\begin{array}{c}
u \\
v \\
w
\end{array}\right]_{\text {Old }}
$$


Neighbor Search

-When a region boundary is reached, what's on the other side?
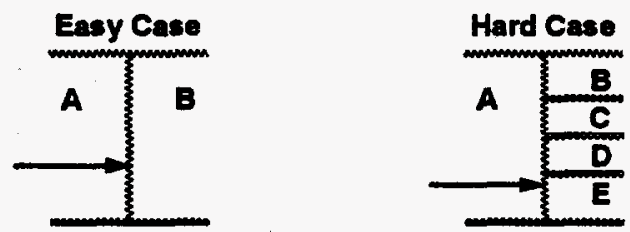

- Most codes build "neighbor lists" during tracking

- for each boundary surface of region, remember list of neighbors

- initially, neighbor lists are empty

- check regions having surface in common until one is found satisfying all sense conditions

- save it

- later, check neighbor lists first, if necessary do search

- Neighbor search is expensive at first, cheap later

- Tracking speeds up as calculation progresses

\section{Miscellaneous Topics}

- Axially varying compositions

- "axcomps", permuted compositions

- "Strong" sense checking \& distance calculations

- "Lost" particles

- Reference points

- Source volumes

- 3-D grid for "site-plot" \& fission-matrix

- Importance regions

- "Slice" plots 


\section{3-D Geometry Schemes - Variations}

Combinatorlal Geometry (e.g., MAGI, MORSE)

- pre-defined primitives ("bodies" -- box, sphere, cone, ...)

- Boolean operations on primitives

- intersection, union, complement operations on bodies

Constructive Solid Geometry (e.g., CSG algorithms for graphics)

- pre-defined primitives (box, sphere, ...)

- general Boolean operations

- intersection, union, complement operations on primitives \& objects constructed from primitives

MCNP Goomotry

- user-defined primitives ("cells" - list of signed surtaces)

- Boolean operations

- intersection \& union of surface half-spaces

- complement of another cell

Sector Goometry (e.g., O5R, RACER)

- user-defined primitives ("sectors" -. list of signed surfaces)

- Boolean operations

- intersection (only) of surface half-spaces

- unions of sectors achieved via "edit-media"

Hole Geometry (e.g., MONK, RACER)

- anything \& everything imaginable (MONK6)

3-D Geometry Scheme Comparison

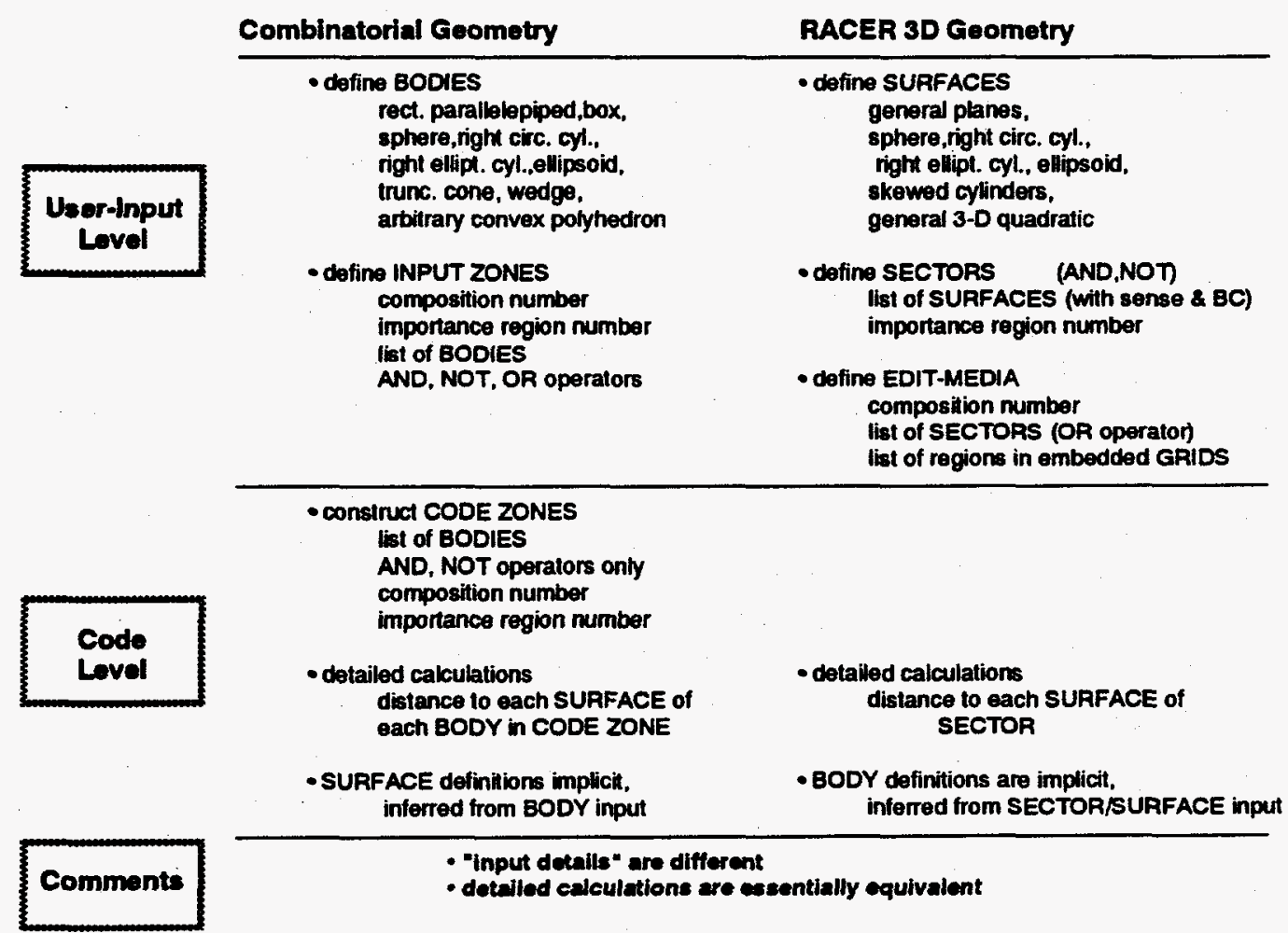




\section{Collision}

\section{Physics}

\section{Epithermal Energy Range}

- continuous energy neutrons \& cross sections

- elastic scattering

- assume free nucleus at rest ( $\left.E \gg k_{B} T\right)$

- conserves kinetic energy and momentum of neutron + nucleus

- scattering from $\mathrm{H}$ bound in $\mathrm{H}_{2} \mathrm{O}$

- accounts for chemical binding

- inelastic scattering

- forms compound nucleus, kinetic energy is not conserved

- multigroup treatment

$\rightarrow$ no upscattering allowed in epithermal range in RACER

\section{Thermal Energy Range - Multigroup Method}

- multigroup neutrons \& cross sections

- only collisions with 'moderators' result in an energy change

- upscattering and downscattering

- $P_{1}$ angular distribution

- scattering from non-moderators is isotropic in the LAB system

\section{Thermal Energy Range - Continuous Energy Method}

- continuous energy neutrons \& cross sections

- scattering handled explicitly for each type

- coherent elastic (Bragg)

- incoherent inelastic $(S(\alpha, \beta))$

- incoherent elastic 


\section{Elastic Scattering From a Free Nucleus at Rest}

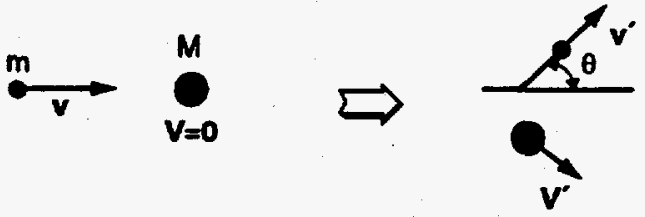

pre-collision

post-collision

LAB Systom

- scattering angle $\theta_{c}$ is sampled in the COM system

- azimuthal angle $\varphi$ is uniformly sampled between 0 and $2 \pi$

- extt energy $E^{\prime}$ is determined by

$$
\begin{aligned}
& E=E \frac{A^{2}+2 A \mu_{c}+1}{(A+1)^{2}} \\
& A=M / m, \mu_{c}=\cos \theta_{c}
\end{aligned}
$$

- LAB scattering angle is determined by

$$
\mu=\frac{1+A \mu_{c}}{\sqrt{A^{2}+2 A \mu_{c}+1}}
$$

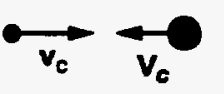

pre-collision

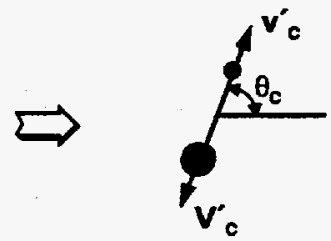

post-collision

coM System

\section{Legendre Expansion of the Scattering Cosine Distribution}

$$
\begin{aligned}
& \sigma\left(\mu_{c}\right)=\text { cross section dependence on the cosine of the scattering angle in the COM system } \\
& f\left(\mu_{c}\right)=\sigma\left(\mu_{c}\right) / \sigma=\text { scattering cosine pof, where } \sigma=\int_{-1}^{1} d \mu_{c} \sigma\left(\mu_{c}\right) \\
& f_{n}=\int_{-1}^{1} d \mu_{c} P_{n}\left(\mu_{c}\right) f\left(\mu_{c}\right)=n^{\text {th }} \text { Legendre moment }
\end{aligned}
$$

$\rightarrow$ Legendre moments are part of the basic cross section data.

Given the moments, the pdf can be reconstructed, i.e.

$$
f\left(\mu_{c}\right)=\sum_{n=0}^{\infty} \frac{2 n+1}{2} f_{n} P_{n}\left(\mu_{c}\right)
$$

In practice, only a finite number of moments are known, so that

$$
f\left(\mu_{c}\right) \sim \sum_{n=0}^{N} \frac{2 n+1}{2} f_{n} P_{n}\left(\mu_{c}\right) .
$$

$\rightarrow$ RACER can utilize Legendre moments up to the $20^{\text {th }}$. 


\section{Selection of the Center-of-Mass Scattering Cosine}

- generate up to (typically) 31 equally-probable step functions

- randomly select one of the 31 angular 'bins'

- uniformly sample scattering cosine between $\mu_{l}$ and $\mu_{i+1}$

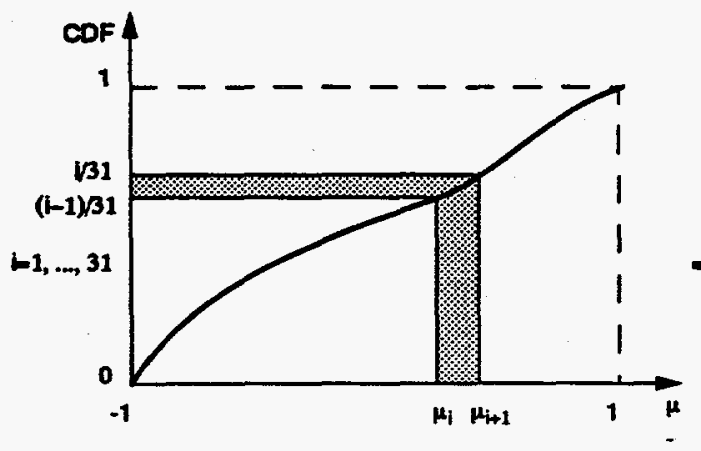

Special cases:

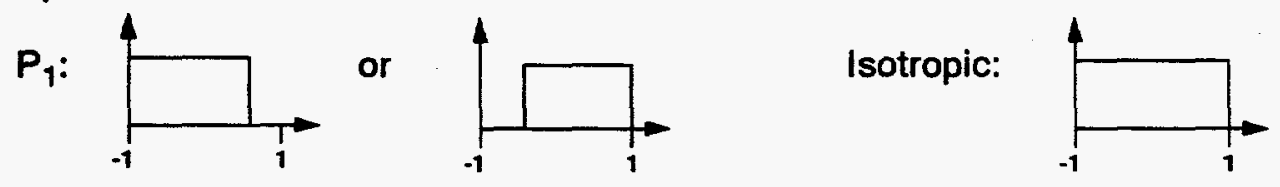

\section{Epithermal Scattering From Bound Hydrogen}

- for scattering from $\mathrm{H}$ in $\mathrm{H}_{2} \mathrm{O}$, the effect of the $\mathrm{H}-\mathrm{O}$ bond must be accounted for

- Nelkin water model

- the proton is treated as a sum of 5 harmonic oscillators

- one transiational mode

- one rotational mode

- three vibrational modes

- the effective temperature is thus given by

$$
k_{B} T_{\text {eff }}=\frac{k_{B} T}{18}+0.0824+0.0176 \operatorname{coth}\left(\frac{0.1025}{k_{B} T}\right)+0.0129 \operatorname{coth}\left(\frac{0.03}{k_{B} T}\right)
$$

- Cady prescription

- PDF for exit energy

$$
\begin{array}{ll}
f_{B O U N D}\left(E \rightarrow E^{\prime}\right)=\delta\left(E-E^{\prime}\right), & E^{\prime}>E \\
f_{B O U N D}\left(E \rightarrow E^{\prime}\right)=\frac{\text { eff }\left(\sqrt{E^{\prime} / k_{B} T_{\text {eff }}}\right)}{E-k_{B} T_{\text {eff }} / 2}, & E^{\prime} \leq E \\
P\left[E^{\prime}>E\right]=\frac{k_{B} T_{\text {eff }}}{E+k_{B} T_{\text {eff }} / 2} .
\end{array}
$$

$\rightarrow$ implemented in RACER using weight modification

- scattering cosine in $L A B$ system is set to average value for bound $H$ downscattering

$$
\mu-\mu_{B O U N D}=\mu_{\text {FREE }}\left[1-\frac{k_{B} T_{\text {eff }}}{E^{2}}+2 \sqrt{\frac{k_{B} T_{\text {eff }}}{\pi E^{\prime}}} \frac{\exp \left(-E / k_{B} T_{\text {eff }}\right)}{\operatorname{erf}\left(\sqrt{E^{\prime} / k_{B} T_{\text {eft }}}\right)}\right]
$$

- scattering cosine in LAB system is set to 1 for upscattering 


\section{Epithermal Inelastic Scattering}

- based on top 25 MUFT groups (need not correspond to supergroups)

- alias sampling from discrete PDF to get exit group $\left(P_{g \rightarrow g^{\prime}}, g^{\prime} \geq g\right)$

- if $g^{\prime} \approx g$,exit energy $E^{\prime}$ is uniformly sampled between $E^{g}$ bot and $E^{g}$ top

- if $g^{\prime}=g, E^{\prime}$ is uniformly sampled between incident energy $E$ and $E_{\text {bot }}$

$\begin{array}{rrr}\text { Grp. } & E_{\text {top }}(0 V & E_{\text {bor }}(0 V \\ 1 & 20,000,000 & 7,788,000 \\ 2 & 7,788,000 & 6,065,000 \\ 3 & 6,065,000 & 4,724,000 \\ 4 & 4,724,000 & 3,679,000 \\ 5 & 3,679,000 & 2,865,000 \\ 6 & 2,865,000 & 2,231,000 \\ 7 & 2,231,000 & 1,738,000 \\ 8 & 1,738,000 & 1,353,000 \\ 9 & 1,353,000 & 1,054,000 \\ 10 & 1,054,000 & 820,800 \\ 11 & 620,800 & 639,600 \\ 12 & 639,600 & 497,600 \\ 13 & 497,600 & 387,700 \\ 14 & 387,700 & 302,000 \\ 15 & 302,000 & 235,200 \\ 16 & 235,200 & 183,200 \\ 17 & 183,200 & 142,600 \\ 18 & 142,000 & 111,100 \\ 19 & 111,100 & 86,520 \\ 20 & 86,520 & 67,380 \\ 21 & 67,380 & 40,870 \\ 22 & 40,870 & 24,790 \\ 23 & 24,790 & 15,030 \\ 24 & 15,030 & 9,119 \\ 25 & 9,119 & 5,531\end{array}$

- history weight is changed to account for $(n, 2 n)$ reactions

- isotropic in LAB or COM systems (user selectable)

\section{Neutron Thermalization}

- neutron energy comparable to $k_{B} T$

$\rightarrow$ upscattering is important

- neutron energy < chemical binding energy

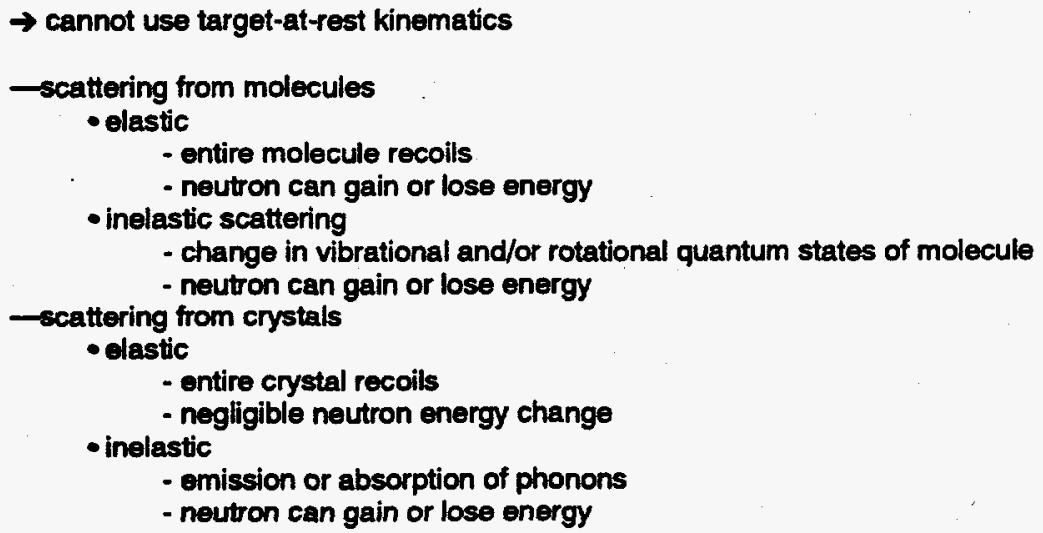

- neutron de Broglie wavelength comparable to interatomic spacing

$\rightarrow$ quantum interference effects-Bragg scattering from crystalline materials 


\section{Multigroup Thermal Treatment}

- typically used for $E<0.625 \mathrm{eV}$

- multigroup method (typically 32)

- all 'isotopes' classified as either moderators or non-moderators

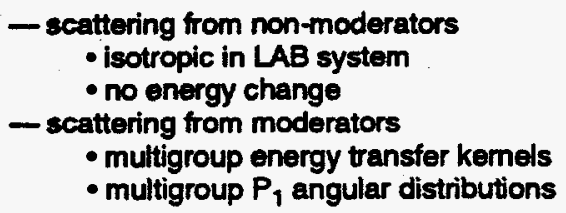

- upscattering allowed, but not back into epithermal range

\section{Scattering From Moderators}

- assume that the double differential scettering cross section $\sigma\left(E \rightarrow E^{\prime}, \mu\right)$ is known

- approximate using a $P_{1}$ expansion

$$
\begin{aligned}
& \sigma\left(E \rightarrow E^{\prime}, \mu\right) \approx \frac{1}{4 \pi} \sigma_{0}\left(E \rightarrow E^{\prime}\right)+\frac{3}{4 \pi} \sigma_{1}\left(E \rightarrow E^{\prime}\right) \mu \\
& \sigma_{0}\left(E \rightarrow E^{\prime}\right)=2 \pi \int d \mu \sigma\left(E \rightarrow E^{\prime}, \mu\right) \\
& \sigma_{1}\left(E \rightarrow E^{\prime}\right)=2 \pi \int d \mu \mu \sigma\left(E \rightarrow E^{\prime}, \mu\right)
\end{aligned}
$$

- average over groups

- total scattering cross section

$$
\sigma^{g}=\left(\int_{E_{g-1}}^{E_{g}} d \int_{0}^{\infty} d E_{\phi}(E) \sigma_{0}\left(E \rightarrow E^{\prime}\right)\right)\left(\int_{E_{g-1}}^{E_{g}} d E_{\phi}(E)\right), \phi=\text { spectral weighting function }
$$

- Oth Legendre moment

$$
\sigma_{0}^{g \rightarrow g^{\prime}}=\left(\int_{E_{g-1}}^{E_{g}} d E_{g^{\prime}-1}^{E_{g^{\prime}}} d E^{\prime} \phi(E) \sigma_{0}\left(E \rightarrow E^{\prime}\right)\right)\left(\int_{E_{g-1}}^{E_{g}} d E_{\phi}(E)\right)
$$

- $1^{\text {st }}$ Legendre moment

$$
\sigma_{1}^{g \rightarrow g^{\prime}}-\left(\int_{E_{g-1}}^{E_{g}} d E \int_{g_{g^{\prime}-1}}^{E_{g^{\prime}}} d E \int d \mu \phi(E) \mu \sigma_{0}\left(E \rightarrow E^{\prime}, \mu\right)\right)\left(\int_{E_{g-1}}^{E_{g}} d E \phi(E)\right)
$$

$\rightarrow$ Note: The upper limit of the integrals over $E^{\prime}$ are assumed to go to infinity for $g^{\prime}=1$. 


\section{Scattering From Moderators (cont.)}

- the P1 approximation to the group-averaged double differential scattering cross section is:

$$
\sigma^{g \rightarrow g^{\prime}}(\mu)=\sigma^{g}\left[\frac{\sigma_{0}^{g \rightarrow g^{\prime}}}{\sigma^{g}}\right] \frac{1}{2 \pi}\left[\frac{1}{2}+\frac{3}{2} \frac{\sigma_{1}^{g \rightarrow g^{\prime}}}{\sigma_{0}^{g \rightarrow g^{\prime}} \mu}\right]
$$

- thus, given a scattering from the moderator in group g:

$$
\begin{aligned}
& \text { - sample the exit group from the discrete poff } \frac{\sigma_{0}^{g \rightarrow g^{\prime}}}{\sigma^{g}}, g^{\prime}=1, \ldots, g \\
& \text { - sample the scattering cosine from the linear pdf } \frac{1}{2}+\frac{3}{2} \frac{\sigma_{1}^{g \rightarrow g^{\prime}}}{\sigma_{0}^{g} \rightarrow g^{\prime}} \mu
\end{aligned}
$$

- uniformly sample the azimuthal angle on $(0,2 \pi)$

\section{Continuous Energy Thermal Treatment}

- typically used for $E<0.625 \mathrm{eV}$

- neutrons and cross sections are continuous in energy

- different scattering types are treated explicitly

- coherent elastic (Bragg)

- incoherent elastic

- incoherent inelastic

- upscattering allowed, but not back into epithermal range 
Scattering From a Single Nucleus at Rest

- asymptotic neutron wave function is given by

$$
\begin{aligned}
& \Psi(r)=\frac{1}{(2 \pi)^{3 / 2}}\left[\Psi_{0}(r)+\Psi_{1}(r)\right] \\
& \Psi_{0}(r)=e^{i k \cdot r}=\text { incident wave function } \\
& \Psi_{1}(r)=f\left(\bar{\Sigma} \cdot \bar{\Omega}^{\prime}\right) \frac{1}{r} e^{i k r}=\text { scattered wave function }
\end{aligned}
$$

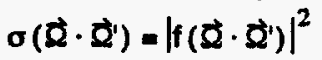

Schrödinger equation

$$
\begin{aligned}
& {\left[-\frac{h^{2}}{8 \pi^{2} m_{r}} \nabla^{2}+V(r)\right] \Psi(r)=E \Psi(r)} \\
& \Psi(r)=\frac{1}{(2 \pi)^{3 / 2}} e^{\mid k \cdot r}-\frac{2 \pi m_{r}}{h^{2}} \int d r \frac{e^{|k| r-r \mid}}{\left|r-r^{\prime}\right|} V\left(r^{\prime}\right) \Psi(r), \quad \begin{array}{l}
m_{r}=r e d u c e d \text { mass, } \\
k=\left(2 \pi \sqrt{2 m_{r} E / h}\right) \AA
\end{array}
\end{aligned}
$$

- make Born approximation

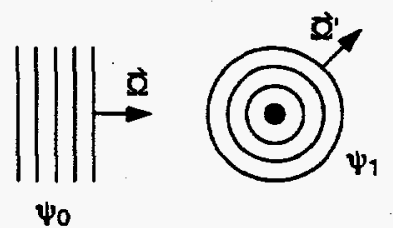

$$
\psi\left(r^{\prime}\right) \leftarrow \frac{1}{(2 \pi)^{3 / 2}} e^{i k \cdot r} \text { in integrand }
$$

- use Fermi pseudopotential

- final result:

$$
V(r)=\frac{h}{m_{r}} \otimes \delta(r), \quad a=\text { scattering length }
$$

$$
\sigma=a^{2}=\sigma_{0}
$$

\section{Collision Physics - Thermal - Scattering Types}

\section{Scattering From Two Nuclei}

- wave function

$$
\psi(r)=\psi_{0}(r)+\psi_{s}(r)
$$

- incident wave function

$$
\psi_{0}(r)=e^{i k z}
$$

- scattered wave function

$$
\begin{aligned}
& \Psi_{s}(r)=\Psi_{1}(r)+\Psi_{2}(r) \\
& \Psi_{1}(r)=-\frac{a_{1}}{r} e^{i k\left(r-\frac{d}{2} \sin \theta \cos \varphi\right)} \\
& \Psi_{2}(r)=-\frac{a_{2}}{r} e^{i k\left(r+\frac{d}{2} \sin \theta \cos \varphi\right)}
\end{aligned}
$$

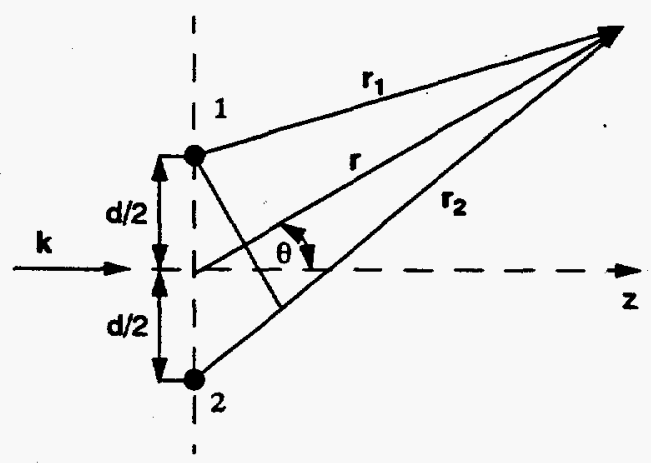

- scattering cross section

$\sigma(\theta, \varphi)=\frac{r^{2}}{2}\left|\Psi_{s}(r)\right|^{2}=\frac{a_{1}^{2}+a_{2}^{2}-2 a_{1} a_{2}}{2}+a_{1} a_{2} 2\left[\cos \left(\frac{k d}{2} \sin \theta \cos \varphi\right)\right]^{2}$

$\rightarrow$ interference effects

$$
\text { - if } \begin{aligned}
a_{1} & =a_{2} \\
& \sigma(\theta, \varphi)=\sigma_{0} 2\left[\cos \left(\frac{k d}{2} \sin \theta \cos \varphi\right)\right]^{2}
\end{aligned}
$$


Scattering From $\mathbf{N}$ Fixed Nuclei

$$
\begin{aligned}
\sigma(\theta, \varphi) & =\frac{1}{N}\left|\sum_{n=1}^{N} a_{n} \theta^{i K \cdot R_{n}}\right|^{2} \\
& =\frac{1}{N} \sum_{n=1}^{N} a_{n}^{2}+\frac{1}{N} \sum_{n=1}^{N} \sum_{m=n}^{N} a_{n} a_{m} e^{i K \cdot\left(R_{n}-R_{m}\right)}
\end{aligned}
$$

assume $a_{n}$ are not correlated with position, then

$$
\begin{aligned}
& \sigma(\theta, \varphi)=\left\langle a^{2}\right\rangle+\frac{1}{N}\langle a\rangle^{2} \sum_{n=1}^{N} \sum_{m=n}^{N} e^{i K \cdot\left(R_{n}-R_{m}\right)} \\
& \langle a\rangle=\frac{1}{N} \sum_{n=1}^{N} a_{n}\left\langle a^{2}\right\rangle=\frac{1}{N} \sum_{n=1}^{N} a_{n}^{2}
\end{aligned}
$$$$
\longrightarrow
$$

rearrange

$$
\sigma(\theta, \varphi)=\sigma_{\text {inc }}+\sigma_{\operatorname{coh}} \frac{1}{N}\left|\sum_{n=1}^{N} e^{i K \cdot R_{n}}\right|^{2}, \quad \sigma_{\text {inc }}=\frac{1}{4 \pi}\left(\left\langle a^{2}\right\rangle-\langle a\rangle^{2}\right) \quad \sigma_{\operatorname{coh}}=\frac{1}{4 \pi}\langle a\rangle^{2}
$$

If $\sigma_{\text {inc }}=0 \rightarrow$ coherent elastic scattering

\section{General Case of Scattering From N Nuclei}

- in general, we have

$$
\begin{aligned}
& \sigma\left(E \rightarrow E^{\prime}, \bar{\Sigma} \rightarrow \bar{\Sigma}^{\prime}\right)=\sigma_{\text {inc }}\left(E \rightarrow E^{\prime}, \overline{\mathbf{\Sigma}} \rightarrow \overline{\mathbf{\Sigma}}^{\prime}\right)+\sigma_{\mathrm{coh}}\left(E \rightarrow E^{\prime}, \overline{\mathbf{\Omega}} \rightarrow \mathbf{\Sigma}^{\prime}\right) \\
& \sigma_{\text {inc }}\left(E \rightarrow E^{\prime}, \vec{\Omega} \rightarrow{\overrightarrow{\Omega^{\prime}}}^{\prime}\right)=\frac{\sigma_{\text {inc }}}{2 h} \sqrt{\frac{E}{E}} \frac{1}{2 \pi} \int_{-\infty}^{\infty} d t \int d r e^{i(K \cdot r-2 \pi \varepsilon t / h)} G_{s}(r, t) \\
& \sigma_{\operatorname{coh}}\left(E \rightarrow E^{\prime}, \bar{\Omega} \rightarrow \bar{\Omega}^{\prime}\right)=\frac{\sigma_{\operatorname{coh}}}{2 h} \sqrt{\frac{E}{E^{\prime}}} \frac{1}{2 \pi} \int_{-\infty}^{\infty} d t \int d r e^{i(K \cdot r-2 \pi \varepsilon t / h)} G(r, t) \\
& G_{s}(r, t)=\frac{1}{N} \sum_{n=1}^{N} \int d r^{\prime}\left\langle\delta\left(r+R_{n}(0)-r^{\prime}\right) \delta\left(r^{\prime}-R_{n}(t)\right)\right\rangle \\
& G(r, t)=\frac{1}{N} \sum_{n=1}^{N} \sum_{m=1}^{N} \int^{\prime} d r^{\prime}\left\langle\delta\left(r+R_{m}(0)-r^{\prime}\right) \delta\left(r^{\prime}-R_{n}(t)\right)\right\rangle \\
& E=E-E^{\prime}, K=2 \pi m\left(v-v^{\prime}\right) / h \\
& S_{8}(K, \varepsilon)=\frac{1}{2 \pi} \int_{-\infty}^{\infty} d t \int d r e^{i(K \cdot r-2 \pi \varepsilon t / h)} G_{s}(r, t), S(K, \varepsilon)=\frac{1}{2 \pi} \int_{-\infty}^{\infty} d t \int d r e^{i(K \cdot r-2 \pi \varepsilon t / h)} G(r, t)
\end{aligned}
$$

- for some materials, we may define

$$
\begin{aligned}
& S_{s}(\alpha, \beta)=k_{B} T e^{\beta / 2} S_{s}(K, \varepsilon), S(\alpha, \beta)=k_{B} T_{\theta}^{\beta / 2} S(K, \varepsilon) \\
& \alpha=\frac{h^{2} K^{2}}{2 M K_{B} T}, \beta=-\varepsilon / k_{B} T \\
& \rightarrow \text { this representation is used for incoherent inelastic scattering }
\end{aligned}
$$




\title{
Tallies
}

\author{
\&
}

\section{Statistical Edits}

\section{Tallies}

- analog Monte Carlo

- each history is assigned a weight' of 1

- final event estimator

- follow each history until it terminates by absorption or leakage

- If termination by leakage, tally 1 leakage

- If termination by absorption, tally

$-\Sigma_{\mathrm{a}, \mathrm{j}} / \Sigma_{\mathrm{a}}$ for all nuclides $\mathrm{j}$ in the region where the absorption occurred

$$
-\boldsymbol{v} \Sigma_{\mathbf{f}} / \boldsymbol{\Sigma}_{\mathbf{a}}
$$

- guarantees that absorptions + leakages $=$ source $+(n, 2 n)$ exactly

- weighted Monte Carlo

- each history is initially assigned a weight $(W)$ of 1

- at each collision:

- employ collision estimator

- tally $w \Sigma_{x, j} / \Sigma_{1}$ for all reactions $x$ and nuclides $j$ in the region where the collision occurred

- collision estimator is usually best for optically thick regions

- reduce the weight by multiplying by the nonabsorption probability, $w \leftarrow w\left(1-\Sigma_{a} / \Sigma_{t}\right)$

$\rightarrow$ survival biasing

— for each fight (collision-to-collision, boundary-to-collision, etc.)

- employ path-length estimator

- tally $w s \Sigma_{x, j}$ for all reactions $x$ and nuclides $j$ in the region ( $s=$ path-length of flight)

- path-length estimator is usually best for optically thin regions

- terminate histories using Aussian Roulette

- better estimator is a linear combination of collision and path-fength estimators

- combining coefficients are chosen to minimize variance

- combining coefficients are edit-quantity-dependent

- absorptions + leakages - source $+(n, 2 n)$ on the average 
Introduction to Statistics

\section{Charactorizing Random Data}

Given a set of $N$ random values $\left\{x_{1}, x_{2}, \ldots, x_{N}\right\}$, one can calculate:

- sample mean

$$
m=\frac{1}{N} \sum_{i=1}^{N} x_{i}
$$

- sample variance

$$
s^{2}=\frac{1}{N-1} \sum_{i=1}^{N}\left(x_{i}-m\right)^{2}
$$

$\rightarrow$ for truly random data with $p d f p(x)$ :

$$
\begin{array}{ll}
\langle m\rangle=E[m]=\mu, & \mu=\int d x \times p(x)=\text { mean } \\
\left\langle s^{2}\right\rangle=E\left[s^{2}\right]=\sigma^{2}, & \sigma^{2}=\int d x(x-\mu)^{2} p(x)=\text { variance }
\end{array}
$$

\section{Introduction to Statistics}

\section{Central Limit Theorem}

As the sample size $\mathrm{N}$ increases, the distribution of the sample mean $\mathrm{m}$ of a sample drawn from almost any distribution approaches a normal distribution with mean $\mu$ and variance $\sigma^{2} / N$.
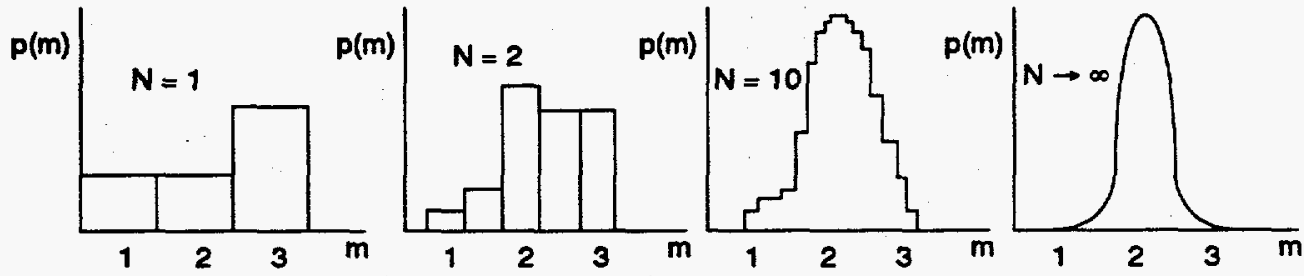

Confidence Intervals

- the $95 \%$ confidence intervals d95 are given by

$$
d_{95}=t_{N-1} s / \sqrt{N}, \quad t_{N-1}=\text { Student's } t \text { factor for } N-1 \text { degrees of freedom }
$$

Given a sample mean $m$, with sample variance $s$, there is a $95 \%$ probability that the population mean $\mu$ lies in the interval $\left[m-d_{95}, m+d_{95}\right]$.

Teat for Normality

- the skewness coefficient $c$ ( $<C>=0$ for normal distribution)

$$
c=\frac{1}{s^{3}} \frac{1}{N-1} \sum_{i=1}^{N}\left(x_{i}-m\right)^{3}
$$

- a confidence interval is doubtful if $c^{2} \geq(1.96)^{2}\left[\frac{6(N-1)(N-2)}{N(N+1)(N+3)}\right]$ (marked with a $=$ in FACED)

- a confidence interval is probably meaningless if $c^{2} \geq(2.81)^{2}\left[\frac{6(N-1)(N-2)}{N(N+1)(N+3)}\right]$ (marked with a "?) 


\section{RACED Output}

\section{Single Estimators}

- RACER supplies RACED with one value of a reaction rate, $x_{i}$, for each of $N$ non-discarded batches $i$

- RACED computes

- the sample mear:

$$
\begin{aligned}
& m=\frac{1}{N} \sum_{i=1}^{N} x_{i} \\
& s^{2}=\frac{1}{N-1} \sum_{i=1}^{N}\left(x_{i}-m\right)^{2}
\end{aligned}
$$

- the sample variance:

- the $95 \%$ confidence interval:

$$
d_{95}=t_{N-1} s / \sqrt{N}
$$

- the skewness coefficient: $\quad c=\frac{1}{N-1} \sum_{i=1}^{N}\left(\frac{x_{i}-m}{s}\right)^{3}$

- RACED outputs the mean along with the $95 \%$ confidence interval and an indication of its uncertainty

\section{RACED Output}

\section{Double Estimators}

- RACER supplies RACED with path-length and collision estimators, $x_{i}$ and $y_{i}$, for each batch $j$

- RACED computes

$$
\begin{array}{ll}
\text { - the sample means: } & m_{x}=\frac{1}{N} \sum_{i=1}^{N} x_{i} \text { and } m_{y}=\frac{1}{N} \sum_{i=1}^{N} y_{i} \\
\text { - the sample variance: } & s_{x}^{2}=\frac{1}{N-1} \sum_{i=1}^{N}\left(x_{i}-m_{x}\right)^{2} \text { and } s_{y}^{2}=\frac{1}{N-1} \sum_{i=1}^{N}\left(y_{i}-m_{y}\right)^{2} \\
\text { - the covariance: } & s_{x y}^{2}=\frac{1}{N-1} \sum_{i=1}^{N}\left(x_{i}-m_{x}\right)\left(y_{i}-m_{y}\right)
\end{array}
$$

- these are used to compute the minimum total variance and corresponding mean

$$
\begin{array}{ll}
\text { - mean: } & m=\alpha m_{x}+(1-\alpha) m_{y} \\
\text { - minimum variance: } & s^{2}=\alpha^{2} s_{x}^{2}+2 \alpha(1-\alpha) s_{x y}^{2}+(1-\alpha)^{2} s_{y}^{2} \\
& \alpha=\frac{s_{y}^{2}-s_{x y}^{2}}{s_{x}^{2}-2 s_{x y}^{2}+s_{y}^{2}}, \text { but set to } 0 \text { if negative and to } 1 \text { if }>1 \\
\text { - the } 95 \% \text { c. } i .: \quad d_{95}=t_{N-1} s / \sqrt{N} \\
\text { - the skewness coefficient is obtained in an analogous manner }
\end{array}
$$

- RACED outputs the mean along with the $95 \%$ confidence interval and an indication of its uncertainty 


\section{RACED Output}

Combination Edits

- combination edits are sums of other edits, i.e.

$$
x_{i}=\sum_{j \in \text { combination }} x_{j i}, y_{i}=\sum_{j \in \text { combination }} y_{j i}
$$

$x_{j i}, y_{j i}=$ estimators of quantity $j$ in batch $i$

- the sums $x_{i}$ and $y_{i}$ are analyzed using the usual procedures for single and double estimators

\section{Ratio Edits}

- ratio edits are ratios of other edits

- ratio edits use a single estimator only

- let $x_{i}$ and $y_{i}$ be batch $i$ single estimator values for the numerator and denominator, resp., of the ratio - RACED computes

$$
\begin{aligned}
& m_{x}=\frac{1}{N} \sum_{i=1}^{N} x_{i} \text { and } m_{y}=\frac{1}{N} \sum_{i=1}^{N} y_{i} \\
& s_{x}^{2}=\frac{1}{N-1} \sum_{i=1}^{N}\left(x_{i}-m_{x}\right)^{2}, s_{y}^{2}=\frac{1}{N-1} \sum_{i=1}^{N}\left(y_{i}-m_{y}\right)^{2}, \& s_{x y}^{2}=\frac{1}{N-1} \sum_{i=1}^{N}\left(x_{i}-m_{x}\right)\left(y_{i}-m_{y}\right)
\end{aligned}
$$

- these are used to obtain

- the mean of the ratio:

$m=m_{x} / m_{y}$

- the variance of the ratio:

$$
s^{2}=\left(\frac{m_{x}}{m_{y}}\right)^{2}\left[\frac{s_{x}^{2}}{m_{x}^{2}}-\frac{2 s_{x y}^{2}}{m_{x} m_{y}}+\frac{s_{y}^{2}}{m_{y}^{2}}\right]
$$

- the $95 \%$ confidence interval: $\quad d_{95}=t_{N-1} s / \sqrt{N}$

\section{RACED Output}

Notes:

- the single estimator procedures are used lf:

- delta tracking is being used

- the bigdepl option is being used (estimator is average of path length and collision estimators)

- for double estimators, separate minimum $\sigma^{2}$ linear combinations are found for each quantity

- energy-integrated values will not in general equal the sum over edit groups

- combination edits will not in general equal the sum of the constituent edits

— ratio edits will not in general equal the ratio of double estimators 


\section{Source Correlation Correction of Confidence Intervals}

- For iterated-source problems, the batch-wise results are correlated

- starting locations for a batch are sampled from the fission sites of the previous batch

- this leads to a bias in the confidence intervals

- For iterated-source problems, RACED optionally applies a correction factor to the confidence intervals

- The true eample varlance can only be found by analyzing results from independent calculations

$m_{j}=$ result from job $j$ (all $\mathrm{J}$ jobs are identical except for random number sequences)

$$
s_{\mu}{ }^{2}=\frac{1}{J-1} \sum_{j=1}^{J}\left(m_{j}-m\right)^{2}, m=\frac{1}{J} \sum_{j=1}^{J} m_{j}
$$

- the expected value of $s^{2}$ is $\sigma_{u}^{2}$

- The apparent sample variance is computed as if the batch-wise results in a single job were uncorrelated

$x_{i j}=$ result from batch $i$ of $j o b j, \quad m_{j}=\frac{1}{N} \sum_{i=1}^{N} x_{i j}$
$s_{M j}^{2}=\frac{1}{N(N-1)} \sum_{i=1}^{N}\left(x_{i j}-m_{j}\right)^{2}$

- the expected value of $s_{\mu \mathrm{j}}^{2}$ is $\sigma_{\mu \mathrm{a}}^{2}$, but $\sigma_{\mu \mathrm{a}}^{2}$ is not equal to $\sigma_{\mu}^{2}$

- An approximate relationship between the true and apparent variances is

$$
\sigma_{\mu}^{2}=\sigma_{\mu a}^{2}\left[1+\frac{2}{N} \sum_{k=1}^{N-1}(N-k) \gamma_{k}\right] \text {, where } \gamma_{k} \text { is the correlation coefficient lag } k
$$

- RACED correction:

- make the approximation $\gamma_{k} \approx \gamma_{k-1}\left(\lambda_{1} / \lambda_{0}\right)$, where $\lambda_{1} / \lambda_{0}=$ dominance ratio (default $=0.9$ )

- approximate the true variance by

$$
s_{\mu}^{2}=\max \left[s_{\mu \mathrm{j}}^{2}\left[1+\frac{2 \hat{y}_{1}}{1-\left(\lambda_{1} / \lambda_{0}\right)}\right], s_{\mu \mathrm{j}}^{2}\right]
$$

Talles and Statistical Edits

\section{Source Normalization Bias}

\section{Source of Bias}

- In a real reactor at steady state, the number of neutrons/generation fiuctuates about a mean value

- In PACER, the number of starting histories per batch is constant

- prevents extinction or overflow of population

- simplifies algorithm

- leads to a bias for eigenvalue problems, if not corrected, since all starting distributions are (unfairly) given equal weights

Bias Correction In RACED

Define:

$$
\begin{aligned}
& K=\left(\prod_{i=1}^{N} k_{i}\right)^{1 / N}=\text { geometric average of batch multiplication factors } \\
& M_{1}=1 ; \quad M_{i}=\left(\prod_{j=1}^{i-1} k_{i}\right) / K^{i-1}=\prod_{j=1}^{i-1} K_{j}, i>1
\end{aligned}
$$

Edit the weighted batch averages

$$
x=\left(\sum_{i=1}^{N} M_{i} x_{i}\right) /\left(\sum_{i=1}^{N} M_{i}\right)
$$

- For ratio edits the numerator and denominator are corrected separately

- The source normalization is normally tumed on for iterated-source calculations

- It is turned off when the bigdepl option is being used

- It can optionally be tumed off for any calculation 


\section{Propagation of Error}

- General Equation

$x=x\left(y_{1}, y_{2}, \ldots, y_{n}\right)$

$\sigma_{x}^{2}=\sum_{i=1 j=1}^{n} \sum_{j=1}^{n} \frac{\partial x \partial x}{\partial y_{i} \partial y_{j}} \sigma_{y_{1} y_{j},}^{2} \quad \sigma_{y_{i} y_{i}}^{2}=\sigma_{y_{1}}^{2}$

examples:

- sum of two quantities

$x=y+z$

$$
\sigma_{x}^{2}=\sigma_{y}^{2}+2 \sigma_{y z}^{2}+\sigma_{z}^{2}
$$

- product of two quantities

$$
x=y z
$$

$$
\sigma_{x}^{2}=z^{2} \sigma_{y}^{2}+2 y z \sigma_{y z}^{2}+y^{2} \sigma_{z}^{2}
$$

- Equation for Uncorrelated quantities

$$
x=x\left(y_{1}, y_{2}, \ldots, y_{n}\right)
$$

$\sigma_{x}^{2}=\sum_{i=1}^{n}\left(\frac{\partial x}{\partial y_{i}}\right)^{2} \sigma_{y_{i}}^{2}$

example:

- difference of two estimators

$$
x=y-z
$$$$
\sigma_{x}^{2}=\sigma_{y}^{2}+\sigma_{z}^{2}
$$

$\rightarrow$ These expressions are in terms of $\sigma^{2}$, but similar relations hold for the square of the confidence intervals. 


\section{Monte Carlo}

\section{Eigenvalue Calculations}

Eigenvalue Problems - Reactor "criticality"

$$
\begin{aligned}
& \Psi(p)=\int \Psi\left(p^{\prime}\right) R\left(p^{\prime} \rightarrow p\right) d p^{\prime}+\frac{1}{K_{e f f}} \int \Psi\left(p^{\prime}\right) F\left(p^{\prime} \rightarrow p\right) d p^{\prime} \\
& \Psi=R \cdot \Psi+\frac{1}{K_{e f f}} F \cdot \Psi
\end{aligned}
$$

Generation Model

$$
\begin{aligned}
& \Psi^{(i+1)}=R \cdot \Psi^{(i+1)}+\frac{1}{K_{e f f}^{(i)}} F \cdot \Psi^{(i)} \\
& \Psi^{(i+1)}=\frac{1}{K_{\text {eff }}^{(i)}}[1-R]^{-1} F \cdot \Psi^{(i)} \quad K_{e f f}^{(i)}=\int F \cdot \Psi^{(i)} d p d p^{\prime}
\end{aligned}
$$

Monte Carlo approach:

- Guess $\Psi^{(0)}, K_{\text {eff }}^{(0)}$

- Follow a "batch" of histories, estimate $\Psi^{(i)}, K_{\text {eff }}^{(i)}$

- Repeat until converged

- Discard initial batches

- Iterate, accumulating scores - until variances are small enough 


\section{Single particle}

- random-walk for particle history

- simulate events, from birth to death

- tally events of interest

\section{Batch of histories ("generation")}

- random-walk for many particle histories

- tally the aggregate behavior
Select source $r, \Omega, E$ randomly

1

Track through geometry,

select collision site $\mathrm{r}$ random/y

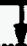

Collision physics analysis, select new $\Omega, E$ randomly

Overall

- timesteps

- geometry changes

- material changes

- fuel depletion

- burnable absorbers

- control rods

$$
\begin{aligned}
& \text { * Loop over timesteps } \\
& \text { - Loop over batches } \\
& \text { - * Loop over histories } \\
& \text { - } \quad \rightarrow \text { random walk } \\
& \bullet \quad \bullet \cdot \\
& \text { - } \rightarrow \text { update Keff \& reaction rates } \\
& \bullet \bullet \\
& \rightarrow \text { compute statistics } \\
& \rightarrow \text { update number densities, ..... }
\end{aligned}
$$

Eigenvalue Calculations - Convergence

Monte Carlo Eigenvalue Calculations

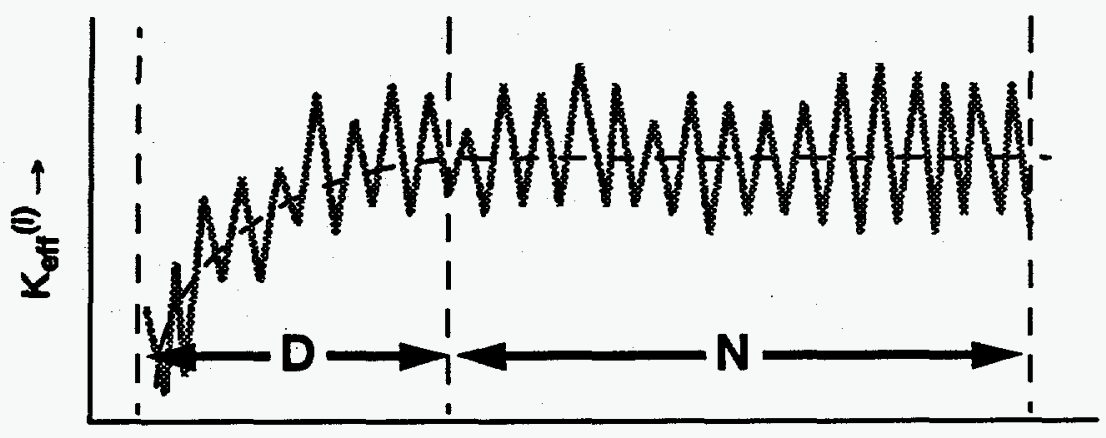

iteration, $\mathbf{i} \rightarrow$

- $D$ = number of initial "contaminated" batches to discard

- $\mathbf{N}=$ number of batches to keep, for averaging results

D \& $\mathbf{N}$ determined by heuristics, not theory:

- should have $\rho^{D}$ " $\sigma_{\text {batch }}$, where $\rho$ is the dominance ratio, \& $\sigma_{\text {batch }}$ is std. dev. in $K_{\text {batch }}$

- should have $\quad N » 25$, so that $\sigma$ 's are small \& reliably estimated

- use larger $\mathbf{N}$ on weekends, ..... 
K-effective - batchwise

$M=1,000$ histories/batch

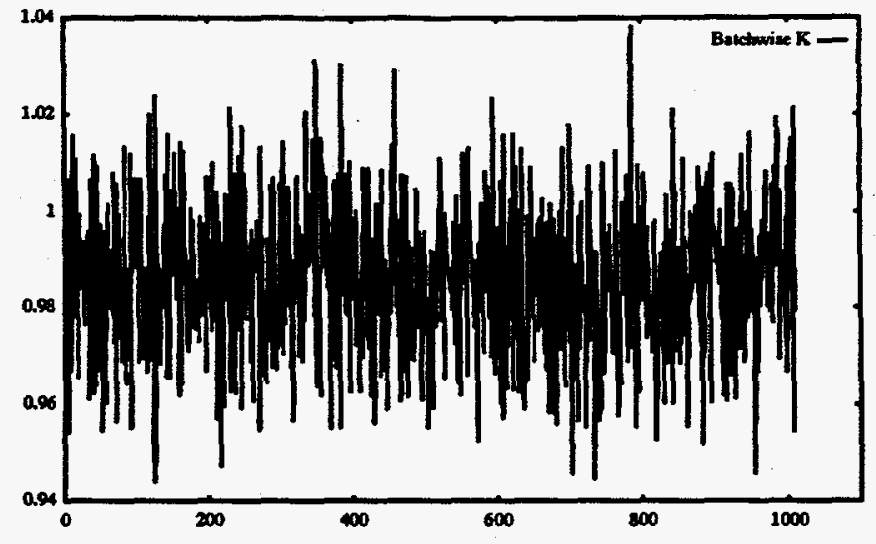

$D=10$ batches discarded

$N=1,000$ batches kept

K-effective - cumulative

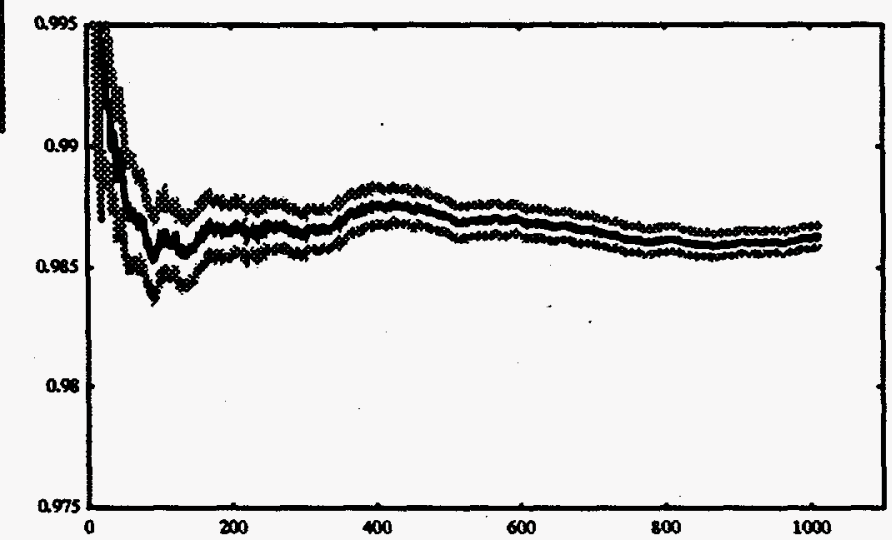

Elgenvalue Calculations - Convergence

K-effective - batchwise

$M=10,000$ histories/batch

$D=10$ batches discarded

$N=100$ batches kept

K-effective - cumulative
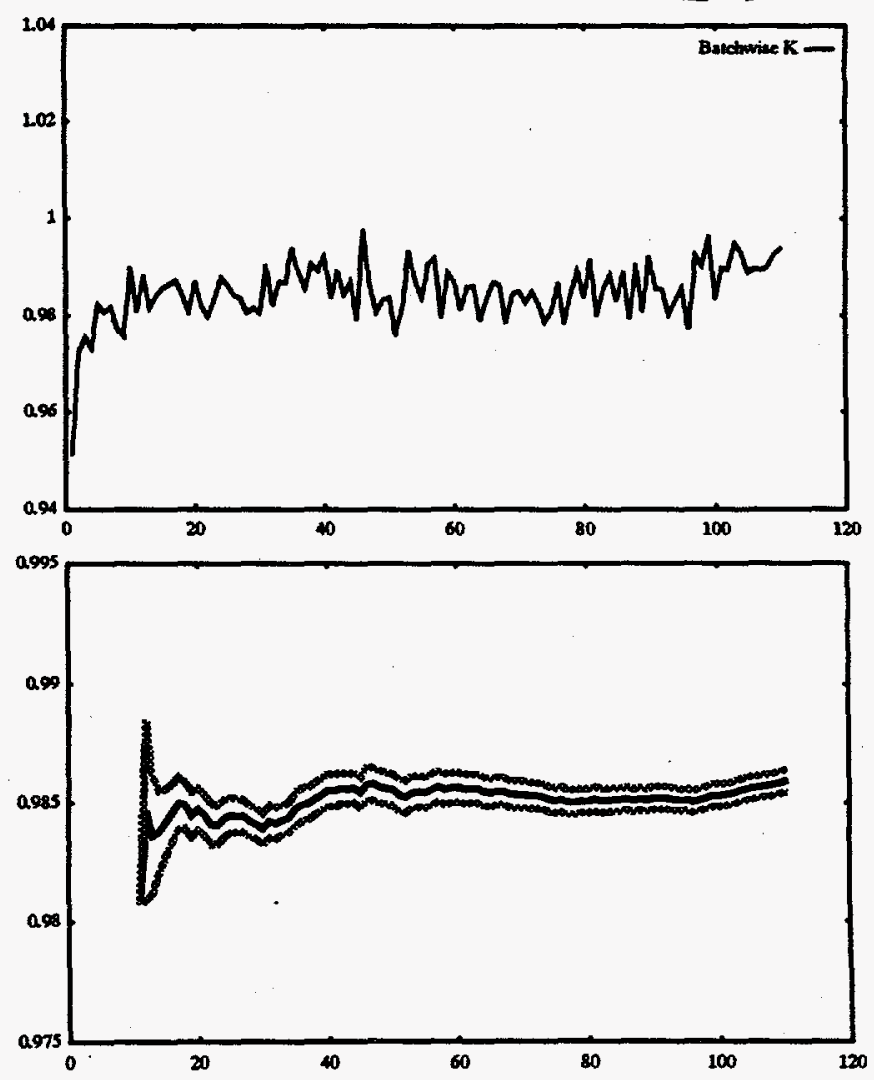
K-effective - batchwise

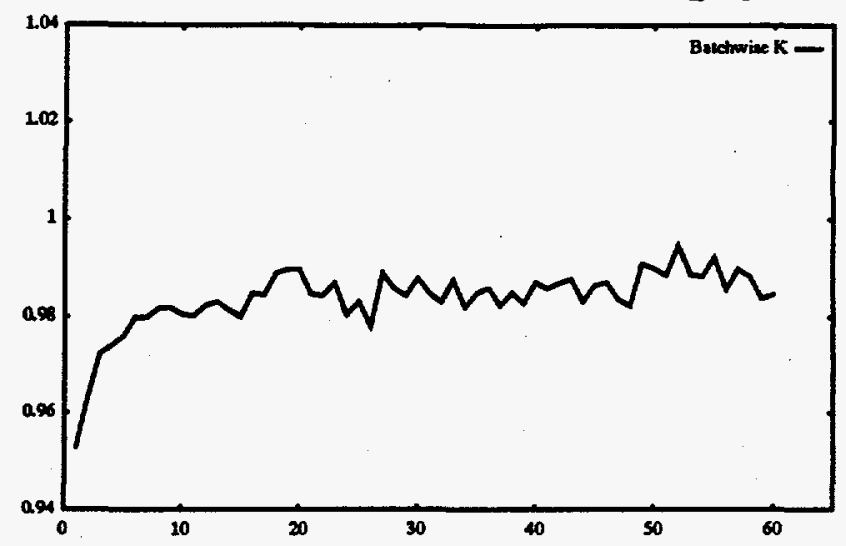

$M=20,000$ histories/batch

$D=10$ batches discarded

$\mathrm{N}=\mathbf{5 0}$ batches kept

K-effective - cumulative

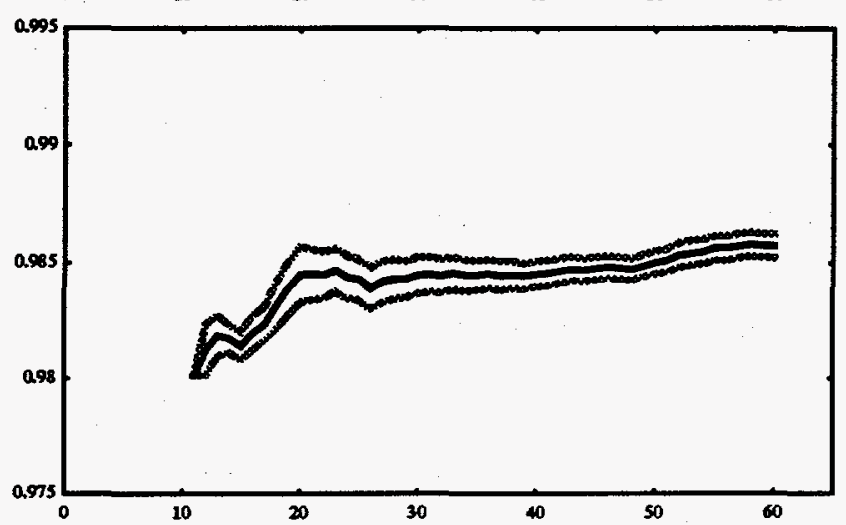

Elgonvalue Calculatlons - Blas - Overview

Bias

- $K_{j}=$ eigenvalue from a single Monte Carlo calculation

- Repeat the calculation $J$ times

(with different random numbers)

- If

$$
\lim _{J \rightarrow \infty} 1 \frac{j}{j} \sum_{j=1}^{j} k_{j}=k_{\text {exact }}
$$

then the Monte Carlo algorithm is unbiased

\section{Eigenvalue Calculations}

- Unaccelerated power method

$$
S^{(i+1)}(r)=\frac{1}{K^{(i)}} \int^{(i)}(r) H(r \rightarrow r) d r
$$

- Typical Monte Carlo algorithm

- follow batch of $\boldsymbol{M}$ histories - store fission sites in a "bank"

- from $\boldsymbol{M}^{\prime}$ banked sites, randomly choose $\boldsymbol{M}$ to start next. batch

- Source renormalization [ $\boldsymbol{M}^{\prime} \rightarrow \boldsymbol{M}$ ] introduces bias

- needed to prevent population explosion or extinction 
Theory

- Discrete M C: $\quad$ Gelbard \& Prael (1974), Brissenden \& Garlick (1986),...

- Continuous M C: ${ }_{2}$ Sutton \& Brown (1991), .....

- bias $=-\frac{\sigma_{k}{ }^{2}}{k_{\text {eff }}} \cdot$ [sum of correlation coeff's between batch $k$ 's]

$$
=-\frac{\sigma_{k}^{2}}{2 k_{e f f}} \sum_{j=1}^{\infty} R_{k k}^{j}
$$

- Smaller M

$\mathbf{M}=$ histories / generation

$\rightarrow$ larger bias in $k_{\text {eff }}$

$\rightarrow$ larger bias in source

\section{Practice}

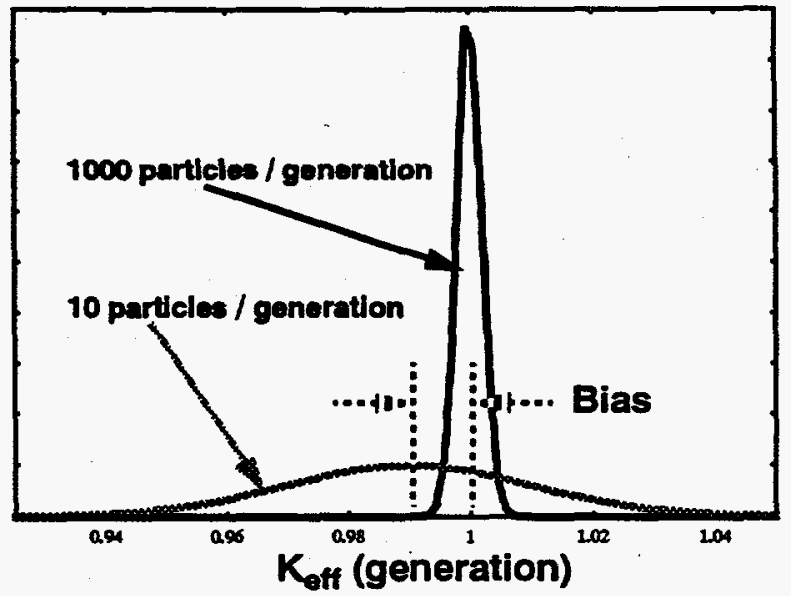

- bigger, faster computers

$\rightarrow$ larger batches

$(M \gg 1,000)$

- negligible bias in $\mathrm{K}_{\text {eff }}$

Eigenvalue vs 1/batch-stze

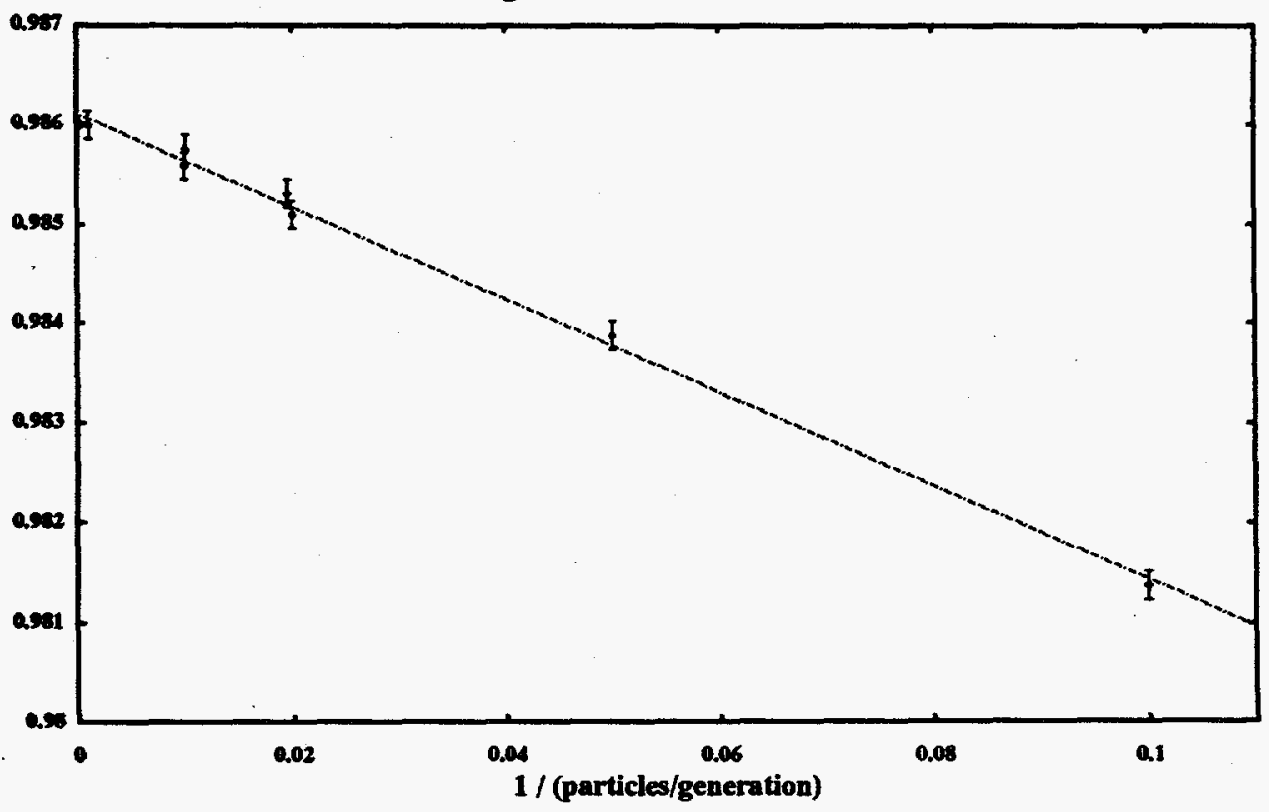


Theory

- Discrete M C: $\quad$ Gelbard, Brown, \& Gu (1993)

- Bias in region source $=-\frac{\sigma_{k} \sigma_{s}}{k_{\text {eff }}} \cdot \begin{gathered}\text { [sum of correlation coeff's between } \\ \text { batch } k \text { \& region source] }\end{gathered}$

$$
=-\frac{\sigma_{k} \sigma_{s}}{2 k_{\text {eff }}} \sum_{j=1}^{\infty} R_{k s}^{i}
$$

- Smaller $M \quad$ ( $M=$ histories / generation )

$\rightarrow$ larger bias in region source

- For reasonable assumptions (verified for many test cases),

$$
\left(\frac{\text { bias }}{\sigma}\right)_{\text {Region Source }}<\left(\frac{\text { bias }}{\sigma}\right)_{\text {Eigenvalue }}
$$

\section{Practice}

- bigger, faster computers $\rightarrow$ larger batches $(M>1,000)$

- negligible bias in eigenvalue $\rightarrow$ negligible bias in region sources

- similar reasoning should (?) apply to other reaction rates

Source shape for various batch sizes

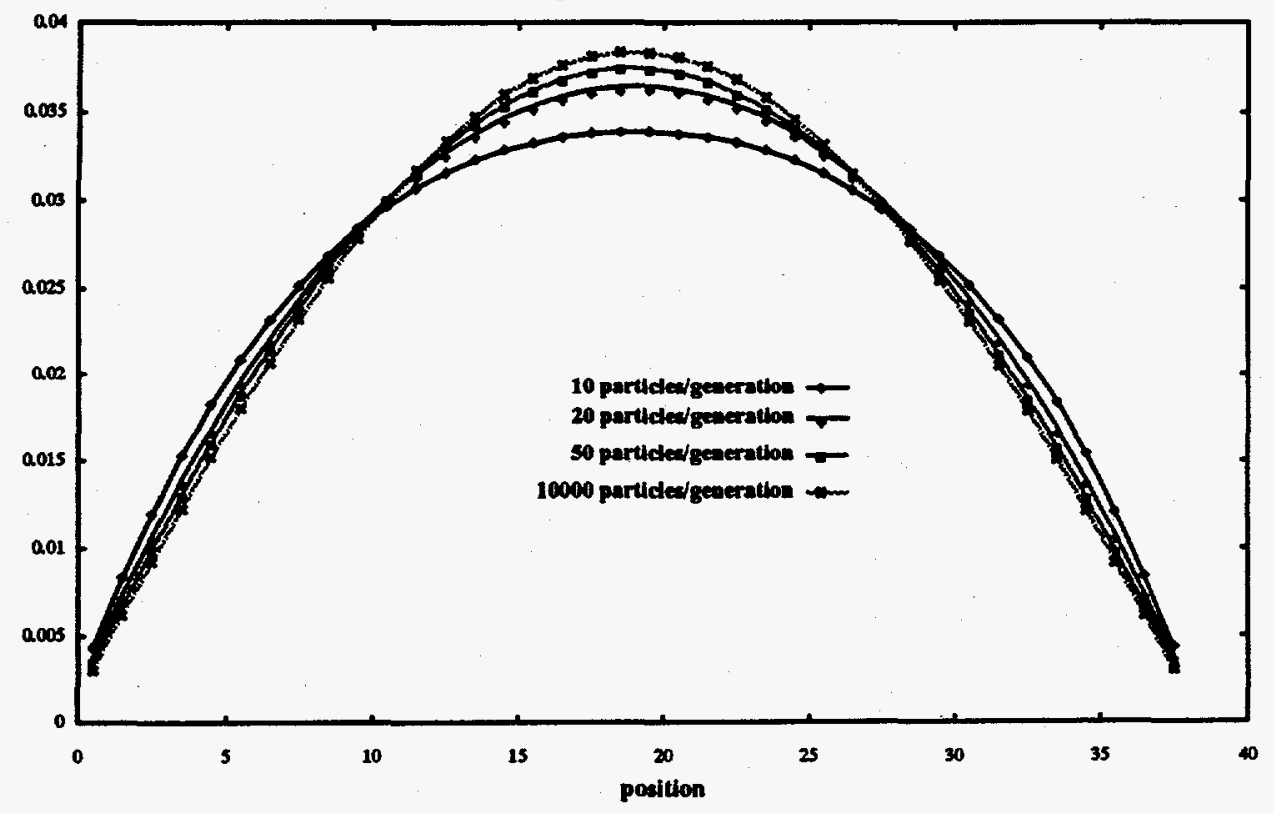




\section{Source Normalization Bias}

Source Normalization Blas

\section{Derivation}

The transport equation can be written in integral form in terms of the source density as

$$
\hat{s}(\mathbf{r})=\frac{1}{\hat{\lambda}} \int d r \hat{s}(\mathbf{r}) H(\mathbf{r} \rightarrow \mathbf{r}) \text {. }
$$

Consider a Monte Carlo calculation where

$$
\begin{aligned}
& S^{(m)}(r)=\sum_{i=1}^{(m)} w_{i}^{(m)} \delta\left(r-r_{i}^{(m)}\right)=\text { fission bank for batch } m \\
& w_{i}^{(m)}=\text { fission production weight at fission site } i \text { of batch } m \\
& r_{i}^{(m)}=\text { location of fission site } i \text { of batch } m .
\end{aligned}
$$

Now, assume that the Monte Carlo algorithm is such that

$$
w_{i}^{(m)}, \sum_{j=1}^{l^{(m)}} w_{j}^{(m)}=\text { probability that a particular history in batch } m+1 \text { will begin at } r_{i}^{(m)}
$$

and thus

$$
s^{(m+l)}(r)=\frac{\int^{d r^{\prime} s^{(m)}(r) H(r \rightarrow r)}}{\int d r^{\prime} s^{(m)}(r)}+\sqrt{N \varepsilon}{ }^{(m)}(r)
$$

where $\varepsilon^{(m)}(r)$ is a random function with zero mean. 
Now define a normalized distribution

$$
s(r)=\left\langle s^{(m)}(r)\right\rangle / N \text {. }
$$

and define a fluctuating component of the Monte Carlo source distribution so that

$$
s^{(m)}(r)=N s(r)+{\sqrt{N} \xi^{(m)}}^{(r)} .
$$

Substitute into

$$
s^{(m+1)}(r)=\frac{\int^{d r s^{(m)}\left(r^{\prime}\right) H\left(r^{\prime} \rightarrow r\right)}}{\int d r s^{(m)}\left(r^{\prime}\right)}+\sqrt{N \varepsilon}{ }^{(m)}(r) .
$$

expand in powers of $1 / \sqrt{\mathrm{N}}$, retaining only terms of order $1 / N$, and ensemble average to obtain

$$
\begin{aligned}
& s(r)=\frac{1}{\lambda} \int \mathrm{dr} s(r) H\left(r^{\prime} \rightarrow r\right)-\frac{1}{N \lambda} \int \mathrm{dr} A(r, r) \int \mathrm{dr} r^{\prime \prime}\left\langle\zeta\left(r^{\prime}\right) \zeta\left(r^{\prime \prime}\right)\right\rangle \\
& \zeta^{(m+1)}(r)=\int^{d r \zeta^{(m)}}(r) A(r, r)+\varepsilon^{(m)}(r) .
\end{aligned}
$$

Now, let

$$
s(r)=\delta(r)+\frac{1}{N} s^{\prime}(r) .
$$

Substitute, retaining term to $1 / N$, to get

$$
s^{\prime}(r)=-\frac{1}{\hat{\lambda}}[1-\Gamma]^{-1} \Gamma \int \mathrm{dr}\left\langle\zeta(r) \zeta\left(r^{\prime}\right)\right\rangle, \text { where } \Gamma f(r)=\frac{1}{\hat{\lambda}} \int \mathrm{dr}\left[H\left(r^{\prime} \rightarrow r\right)-\hat{s}(r)\right] f\left(r^{\prime}\right) .
$$

\section{Source Normalization Bias}

Now, define the eigenvalue bias as

$$
\Delta \lambda=\lambda-\hat{\lambda}=\frac{1}{N} \int \mathrm{drs}^{\prime}(\mathbf{r}) \text {. }
$$

and we find that

$$
\begin{aligned}
\Delta \lambda & =\frac{1}{N \hat{\lambda}} \int \mathrm{dr}[1-\Gamma]^{-1} \Gamma \int \mathrm{dr} r^{\prime}\left\langle\zeta(r) \zeta\left(r^{\prime}\right)\right\rangle \\
& =\frac{1}{N \hat{\lambda}} \int \mathrm{dr} \sum_{j=1}^{\infty} \Gamma^{J} \int^{\mathrm{d} r^{\prime}}\left\langle\zeta(r) \zeta\left(r^{\prime}\right)\right\rangle \\
& =-\frac{1}{N \hat{\lambda}} \int \mathrm{dr} \sum_{j=1}^{\infty} \int \mathrm{dr}^{\prime}\left\langle\zeta^{(m+i)}(r) \zeta^{(m)}\left(r^{\prime}\right)\right\rangle .
\end{aligned}
$$

Finally, use

$$
\frac{1}{N} \int^{d r s}{ }^{(m)}(r)=\frac{1}{N} \int d r\left[N s(r)+\sqrt{N} \zeta^{(m)}(r)\right]
$$

so that

$$
\lambda^{(m)}-\hat{\lambda}=\frac{1}{\sqrt{N}} \int^{d r \zeta^{(m)}}(r)
$$

and

$$
\left.\Delta \lambda=-\frac{1}{\hat{\lambda}} \sum_{j=1}^{\infty}\left\langle\left(\lambda^{(m+j)}-\hat{\lambda}\right)\left(\lambda^{(m)}-\hat{\lambda}\right)\right\rangle=\text { (sum of all eigenvalue covariances }\right) /(\text { true eigenvalue })
$$

$\rightarrow \quad$ For RACER, and the number of histories/batch currently used for design calculations, the bias appears to be completely negligible. 


\section{MacMillan's Correction}

The Monte Carlo iterative scheme may be represented as

$$
s^{(m+1)}(r)=N \frac{\int d r^{\prime} S^{(m)}(r) H(r \rightarrow r)}{\int d r^{\prime} S^{(m)}(r)}+\sqrt{N \varepsilon}{ }^{(m)}(r) .
$$

The ideal (unbiased scheme), would be

$$
\hat{s}^{(m+1)}(r)=\frac{\int \mathrm{dr} \hat{s}^{(m)}\left(r^{\prime}\right) H\left(r^{\prime} \rightarrow r\right)}{\hat{\lambda}}+\sqrt{N \varepsilon}{ }^{(m)}(r),
$$

where the number of histories/batch can fluctuate, and where the true eigenvalue $\hat{\lambda}$ is unknown.

Comparing the two operators, we can see that they differ by the factor

$$
\frac{\operatorname{drs}^{(m)}\left(r^{\prime}\right) / N}{\hat{\lambda}}=\frac{\lambda^{(m)}}{\hat{\lambda}} \text {. }
$$

MacMillan's scheme approximates this by

$$
\frac{\lambda^{(m)}}{\left[\prod_{m=1}^{M} \lambda^{(m)}\right]^{1 / m}}
$$




\section{Variance Reduction}

Variance Reduction

Stratified Sampling

Consider the integral

$$
G=\int_{0}^{1} d x g(x) \text {. }
$$

The Monte Carlo evaluation is

$$
G=\frac{1}{N} \sum_{n=1}^{N} g\left(\xi_{n}\right)
$$

with variance

$$
\sigma^{2}=\frac{1}{N}\left[\int_{0}^{1} \mathrm{dxg}^{2}(x)-\mathrm{G}^{2}\right]
$$

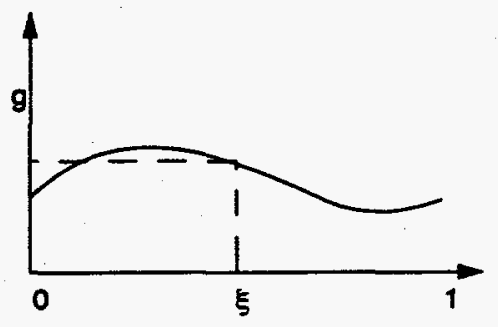

\section{Strattiled sempling:}

- divide domain into $M$ intervals $m$

- perform $N_{m}$ trials in each interval $\left(\sum_{m} N_{m}=N\right)$

- compute the integral

$$
\begin{aligned}
& G_{m}=\frac{\Delta x_{m}}{N_{m}} \sum_{n=1}^{N_{m}} g\left(x_{m-1}+\xi_{n} \Delta x_{m}\right) \\
& G=\sum_{m=1}^{M} G_{m}
\end{aligned}
$$

- the variance is

$$
\sum_{m=1}^{M} \frac{1}{N_{m}}\left[\Delta x_{m} \int_{x_{m-1}}^{x_{m}} d x g^{2}(x)-G_{m}^{2}\right] \leq \sigma^{2}
$$

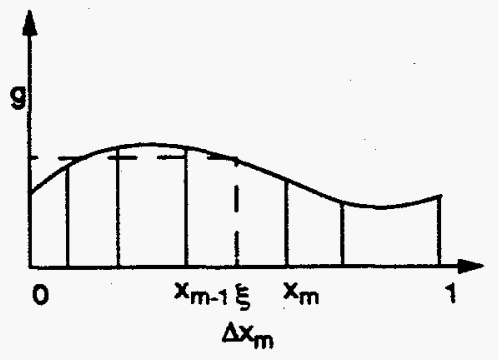


Importance Sampling

Conslder the function $g(x)$ and PDF $p(x)$ defined on the interval $[a, b]$ :

- the mean is

$$
\langle g\rangle-\int_{a}^{b} d x p(x) g(x)
$$

- the variance is

$$
\begin{aligned}
& \sigma^{2}=\left\langle g^{2}\right\rangle-\langle g\rangle^{2} \\
& \left\langle g^{2}\right\rangle=\int_{a}^{p} d x p(x) g^{2}(x)
\end{aligned}
$$

Now, suppose we use instead the PDF $q(x)$ to chose the random numbers on $[a, b]$ :

- assign each trial a "weight" $w(x)=p(x) / q(x)$

- the mean is unchanged

$$
\int_{a}^{b} d x q(x) w(x) g(x)=\int_{a}^{b} d x p(x) g(x)=\langle g\rangle
$$

- the variance is

$$
\begin{aligned}
& \dot{\sigma}^{2}=\int_{a}^{b} d x q(x) w^{2}(x) g^{2}(x)-\left[\int_{a}^{b} d x q(x) w(x) g(x)\right]^{2} \\
& =\int_{a}^{b} d x q(x)\left[\frac{p(x)}{q(x)} g(x)-\langle g\rangle\right]^{2}
\end{aligned}
$$

- if $q(x)$ is chosen to be $p(x) g(x) /\langle g\rangle$ then the variance will be zero (however, $\langle g\rangle$ is not known)

$\rightarrow$ To minimize the variance, choose q so that $\mathrm{pg} / \mathrm{q}$ is approximately constant. Sample more in the "important" regions.

Varlance Reduction

\section{Splitting and Rouletting}

Goal: Track histories that will contribute significantly to the desired results, and don't track those that will not-but do so in a way that is unbiesed.

- define a minimum ( $\left.W_{\text {low }}\right)$, "average" ( $\left.w_{\text {ave }}\right)$, and maximum weights $\left(w_{\text {hi }}\right)$ for each region \& energy range

- following each boundary crossing and collision

- if the history weight $(w)$ is less than $w_{\text {low }}$

- roulette

- terminate the history with probability $1-w / w_{a v e}$

- if it survives, increase the weight to $w_{\text {ave }}$

- If $w$ is greater than $w_{\text {hi }}$

- split

- replace the original history with $[w / w$ ave $\rfloor$ new histories

- create yet another new history with probability $\left(w / w_{\text {ave }}\right)-\left\lfloor w / w_{\text {ave }}\right\rfloor$

- assign the new histories each a weight of $w_{\text {ave }}$

- rouletting increases the variance

- splitting reduces the variance

- splitting requires additional storage

- if done property, the use of splitting and rouletting decreases the cpu time required to achieve a variance 


\section{Additlonal Variance Reduction Methods}

- survival biasing

Instead of terminating upon an absorption, always scatter with reduced weight

- exponential transform

modify total cross section as $\Sigma_{e x}=\Sigma_{t}\left[1-p \vec{d} \cdot \vec{\Sigma}_{0}\right]$

$0 \leq p<1$ = adjustable parameter

d = neutron direction

$\mathbf{D}_{0}=$ preferred direction

- correlated sampling

maintain some correlation (e.g., random number sequence) between unperturbed \& perturbed calcs.

Importance Sampling

Consider the fixed source problem

$\Psi(R)=\int \mathrm{d} R^{\prime} K\left(R^{\prime} \rightarrow R\right) \Psi\left(R^{\prime}\right)+S(R)$

$\mathrm{X}=\int \mathrm{dR} \Psi(R) \times(R)$

$\Psi(R)=$ collision density at phase-space point $\boldsymbol{R}$

$S(R)=$ first-flight collision density due to fixed sources

$X=$ desired functional (e.g., reaction rate)

$\boldsymbol{X}(\boldsymbol{R})=$ contribution to functional due to a collision at $\mathbf{R}$

Decompose the kernel

$K\left(R^{\prime} \rightarrow R\right)=\left(1-\alpha\left(R^{\prime}\right)\right) K\left(R^{\prime} \rightarrow R\right)$

$\alpha(R)=$ absorption probability at $R$

$k\left(R^{\prime} \rightarrow R\right)=$ normailized kernel

Now, define altered quantities

$\bar{\alpha}(R)=x(R) / \psi^{\dagger}(R)$

$\bar{k}\left(R^{\prime} \rightarrow R\right)=k\left(R^{\prime} \rightarrow R\right) \psi^{\dagger}(R) / \int \operatorname{dRk}\left(R^{\prime} \rightarrow R\right) \psi^{\dagger}(R)$

$\bar{S}(R)=S(R) \psi^{\dagger}(R) / \int \mathrm{dRS}(R) \psi^{\dagger}(R)$

where the adjoint function $\psi^{\dagger}(\boldsymbol{R})$ satisfies

$\psi^{\dagger}(R)=\int d R^{\prime} K\left(R \rightarrow R^{\prime}\right) \psi^{\dagger}\left(R^{\prime}\right)+x(R)$

$\rightarrow$ A Monte Carlo calculation using the altered quantities (and with appropriate weights) produces the correct result with eveny history! of course, the adjoint is never exactly known. 


\section{Multiplied Fixed-Source}

\section{Calculations}

Physical Problem: subcritical system with an extraneous source

RACER Mothod:

- total number of histories/batch $=$ constant $=N$

- $N=S+F$

$S=$ number of histories from fixed-source

$F=$ number of histories from induced fission source

$S$ and $F$ may vary from batch to batch

- $\langle F\rangle=k N$

- $(S)=(1-k) N$

- $k=$ (expected \# of neutrons due to fission)/(total \# of neutrons)

RACER Algorithm:

1) first batch: $F_{1}=0 ; S_{1}=N$

2) estimate $k$

- if in discarding stage, $k_{i}=F_{i} / N$

- otherwise, use cumulative value

3) subsequent batches

$$
\begin{aligned}
& F_{i+1}=\left\lfloor k_{i} N+\xi\right\rfloor \\
& \mathbf{S}_{i+1}=N-F_{i+1} \\
& \xi=\text { random number on }(0,1)
\end{aligned}
$$

- Note: the spectra used for the fixed and fission sources may be different. 


\section{Vector \& Parallel}

\section{Monte Carlo}

Trends in Computing Technology

- Commodity chips:

- CPU power

- Memory density

- Supercomputers:

- UNIX operating systems + networking $\rightarrow$ distributed computing

Need to reduce Monte Carlo uncertainties by $2 x$ ?

- Wait 3-4 years for new computer

or

- Use parallel \&/or vector processing now

\section{Alternatives}

- CRAY Supercomputers

- Moderately Parallel computers

- Workstation Network $\rightarrow$ real supercomputing

$\rightarrow$ cheap supercomputing (??)

$\rightarrow$ free supercomputing (??) 
Scientific Computations - State-of-the-Art

Supercomputer Advances:

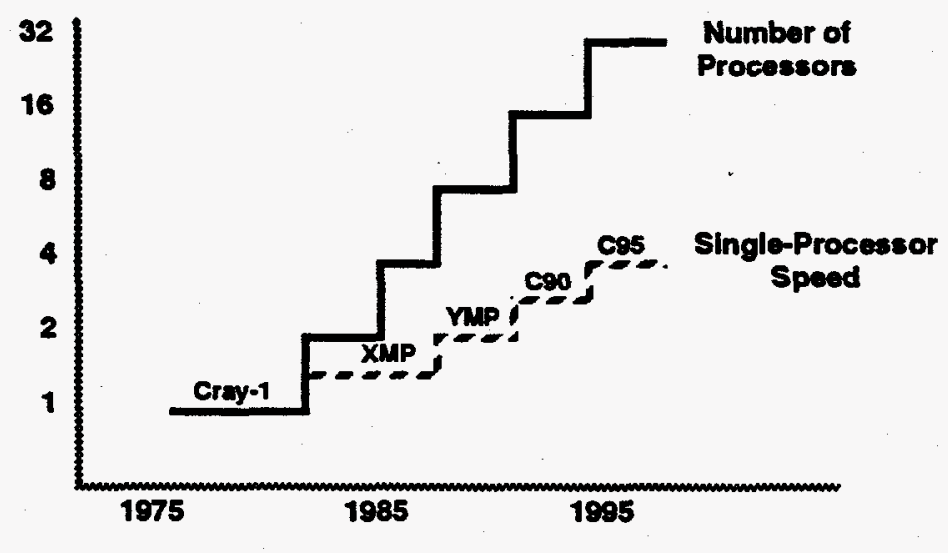

Single-Processor Speed: $\quad 20$ yrs $\Rightarrow 4 X$

Number of Processors: $\quad 20$ yrs $\Rightarrow 32-64 X$

$\Rightarrow$ Parallel \& vector processing are now "routine"

\& necessary for high-performance computing

Vector \& Parallel Monte Carlo - Introduction

Characterize computers by:

- CPU:

scalar, superscalar, pipelined, vector, RISC, CISC,

- Memory:

shared, distributed, cache, banks, bandwidth, .....

- Interconnects: bus, switch, ring, grid, .....

Basic types, with examples:
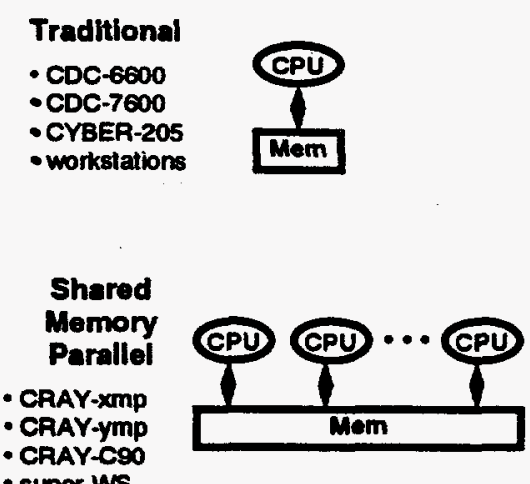
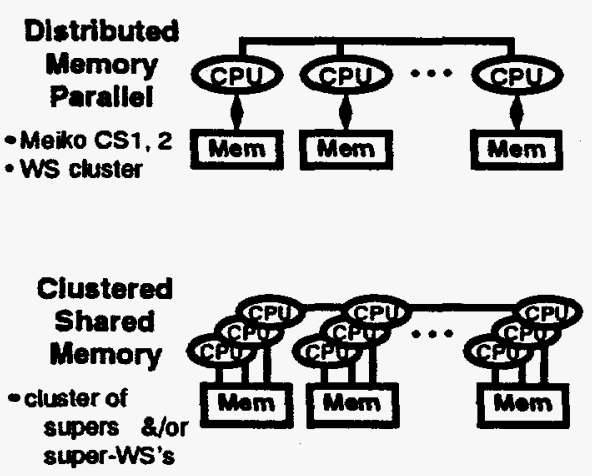
Particle transport Monte Carlo is naturally parallel

- Fixed-source problems: each particle in problem is independent

- Eigenvalue problems: each particle in generation is independent

$\Rightarrow$ Particle histories can be analyzed in parallel

Monte Carlo is often the first use for advanced computers

- Easy to port - compact coding, little $1 / 0$, simple parallel algorithm

- Flexible - independent histories on each node

- Big payoff - bigger \& faster calculations

Computational considerations

- Expensive - hours / days / weeks of computing

- Compact - moderate memory size

- CPU-intensive - very little $1 / 0$ or communications

$T$ (computation) $\gg T($ communications )

Vector \& Parallal Monte Carlo - Vector Processing

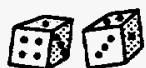

[168]

\section{Vector Processing}

- Fortran

$$
\begin{aligned}
& \text { do } j=1, L \\
& a(j)=b(j)+c(j) \\
& \text { enddo }
\end{aligned}
$$

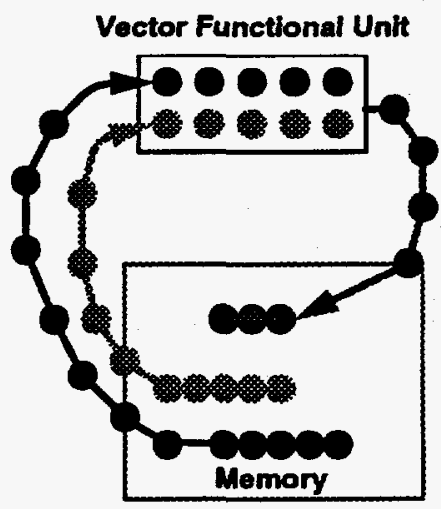

$$
T_{\text {vector }}=t_{\text {startup }}+L \cdot t_{\text {operation }}
$$

Memory

- Speedup

$$
s=T_{\text {scalar }} / T_{\text {vector }}=\frac{L \cdot t_{\text {scalar-op }}}{t_{\text {startup }}+L \cdot t_{\text {vector-op }}} \Rightarrow \frac{t_{\text {scalar-op }}}{t_{\text {vector-op }}}
$$

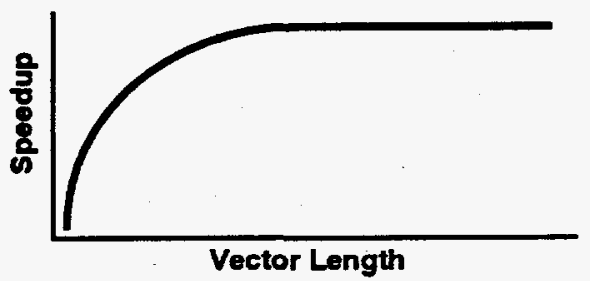




\section{Vector Operations}

- Gather - form a contiguous vector from data in arbitrary locations

$$
\begin{aligned}
& \text { do } j=1, L \quad b 0: \quad 7,5,3,6,1,14 \\
& a(j)=b(i(j)) \\
& \text { i0: } \quad 4,1,2,4,4 \\
& \text { enddo } \quad a 0: \quad 6,7,5,6,6
\end{aligned}
$$

- Scatter - disperse vector data to arbitrary locations

$$
\begin{aligned}
& \text { do } j=1, L \quad \text { b0: } 1,2,3,4 \\
& a(i(j))=b(j) \\
& \text { i0: } 4,1,2,5
\end{aligned}
$$

\section{Vector Operations}

- Mask - either/or selection of data from two vectors

$$
\begin{aligned}
& \text { do } j=1, L \\
& \text { if( test }(0)) \text { then } \\
& \text { test0: } T, \quad T, F, F, . T \\
& \text { else } \\
& c(j)=a(j) \\
& \text { a): } \quad 0,1,2,3,4 \\
& \text { endif } \\
& c(j)=b(i) \\
& \text { b0: } 5,6,7,8,9 \\
& \text { enddo } \\
& \text { c0: } 0, \quad 1,7,8,4
\end{aligned}
$$

- Compressed Index Generation - find the indices of selected items in a vector

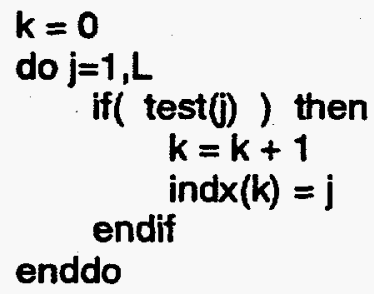


The Tally Loop (scalar)

- Indexed accumulation

$$
\begin{aligned}
& \text { do } j=1, L \\
& \operatorname{sum}(i(j))=\operatorname{sum}(i(j))+r(j) \\
& \text { enddo }
\end{aligned}
$$

- Used to tally particle scores into bins, for overall results

- Tally operations account for 1-10\% of time

- Not readily vectorized (some tricks for Cray-C90)

\section{Writing Efficient Vector Coding}

- Clean loops - structure \& indent (Good-looking code runs faster!)

- Innermost loop should be the vector loop

- Avoid IF tests, unless strictly "either/or"

- Never use "GO TO" statements

- No subroutine calls

- No user-defined function calls

- No recursion (ie, forward-stores or backward-fetches)

- Timing estimates:

- Count all operations inside loop, including both branches for IF's.

- Multiply by vector length \& clock cycle time.

— Measure. If much different from estimate, find out why ! 


\section{Amdahl's Law}

- If a computation has fast (vector) \& slow (scalar) components, the overall calculation time will be dominated by the slower component

- Speedup $=\frac{1}{(1-f)+f / R}, \quad$ where $\begin{aligned} & f=\text { fraction vectorized } \\ & R=\max \text { speedup from vector }\end{aligned}$

\begin{tabular}{|c|c|c|c|c|c|c|c|}
\hline & for & $=10$ & & & for & $=\infty$ & \\
\hline 1 & s & 1 & $\mathbf{s}$ & 1 & s & $f$ & $\mathbf{s}$ \\
\hline $\begin{array}{l}20 \% \\
40 \%\end{array}$ & $\begin{array}{l}1.2 \\
1.6 \\
0 ?\end{array}$ & $\begin{array}{l}90 \% \\
95 \%\end{array}$ & $\begin{array}{l}5.3 \\
6.9\end{array}$ & $\begin{array}{l}20 \% \\
40 \% \\
\end{array}$ & $\begin{array}{l}1.3 \\
1.7 \\
0.5\end{array}$ & $\begin{array}{l}90 \% \\
95 \% \\
\end{array}$ & $\begin{array}{r}10 \\
20 \\
\end{array}$ \\
\hline $\begin{array}{l}60 \% \\
80 \%\end{array}$ & $\begin{array}{l}2.2 \\
3.6\end{array}$ & $\begin{array}{l}99 \% \\
99.5 \%\end{array}$ & $\begin{array}{l}9.2 \\
9.6\end{array}$ & $\begin{array}{l}60 \% \\
80 \%\end{array}$ & $\begin{array}{l}2.5 \\
5\end{array}$ & $\begin{array}{l}99 \% \\
99.5 \%\end{array}$ & $\begin{array}{l}100 \\
200\end{array}$ \\
\hline
\end{tabular}

- For effective vector performance, must vectorize everything !

Vector \& Parallal Monte Carlo - Vectorization

\section{Vectorized Monte Carlo}

- Monte Carlo

Simulate neutron behavior by random-walk.
Select source $r, \Omega, E$ randomly

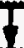

Track through geometry. select collision site $\mathrm{r}$ randomly

$$
1
$$

Collislon physics analysis,

select new $\Omega, E$ randomly

- Conventional Monte Carlo

Analyze many events for one neutron, repeat for other neutrons

- Vectorized Monte Carlo

Analyze many neutrons for one event, répeat for other events

$\Rightarrow$ Event-based algorithms developed by Kalos, Brown/Martin, Bobrowicz 


\section{Monte Carlo is difficult to "vectorize"}

- Branching, data retrieval, \& arithmetic operations vary for each particle, depending on location, type of collision, code options, etc.

- Typically, $\sim 1 / 3$ of essential Fortran statements are IF-tests, which inhibit vectorization

- Not useful: - "automatic vectorizers"

- syntactic hand-vectorization by programmers

[ In early 1980s, LANL tried each approach with monp $\rightarrow 2 X$ slower]

\section{Method for Vectorizing Monte Carlo}

1. Use supercomputer with vector hardware for data-handling

2. Deliberate $\&$ careful development

3. Restructure the database

4. Restructure \& rewrite the Monte Carlo code

Vector \& Parallel Monte Cario - Vectorization

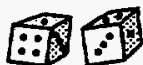

Method for Vectorizing Monte Carlo

1. Use supercomputer with vector hardware for data-handling

- Only $-40 \%$ of operations are floating-point arithmetic $(*,+,-, l$, sqrt $)$

- 40-60\% of operations involve data-handling, indexing, selection, .....

- Must have hardware support for data-handling (gather, scatter, mask, compressed index, ...)

2. Deliberate \& careful development

- Start small, with fow options

- No committees !!!

- Focus effort on total vectorization

- Build gradually, restructuring as needed for new features

- Debugging is extremely difficult - test everything, separately \& integrated

3. Restructure the database

- Unified data formats, with no special cases

- Arrange for simple \& logical direct addressing using vector gather operations

- Use some new (but equivalent) physics, if necessary

4. Restructure \& rewrite the Monte Carlo code

- "Top-down" development, based on event-driven algorithm

- Use some new (but equivalent) physics, if necessary

- Avoid rejection methods for random sampling

- Vectorize" the If-tests by data motion, extra computation, or new algorithms 


\section{Vectorizing IF-tests}

In Monte Carlo codes, IF-tests arise in the context of:

$$
\text { implicit loops, conditional coding, code options }
$$

\section{Implicit loops}

- Logic of the form "loop UNTI ....."

- Usually coded as "IF ..... GOTO" \& backward branch, instead of "DO ....."

- Number of passes is generally not known in advance

- Some particles satisfy the exit conditions on first pass, others take many passes

- Vectorize by:

- Data motion - rearrange the particle data after each pass (eg, event-driven algorithm)

- Extra computation - dummy ops on "finished" particles till all are done

- Different math/physics - eliminate implicit loop (eg, direct sampling instead of rejection)

\section{Conditional coding}

- Selective operations of some particles, but not others

- Vectorize by:

- Gather / Operate / Scatter

- Rearrange selective ops into series of "either/or" ops using vector masks

- Generalized equations, without special cases

\section{Code options}

- Easy - one test/branch for all particles

\section{Monte Carlo - Vectorization}

\section{Conventional Algorithm}

histor
1
2
3
4
5

event sequence

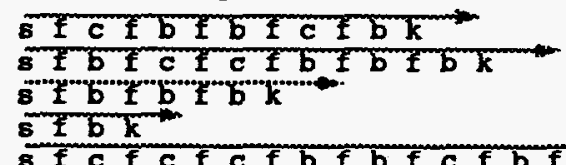

\section{Event-Based Algorithm}

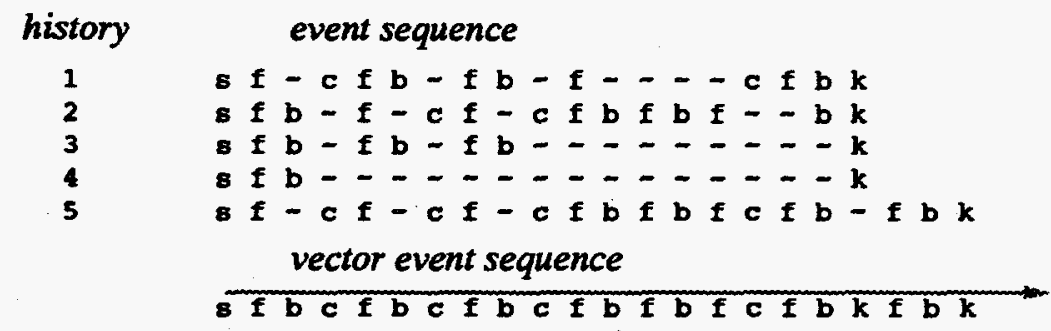




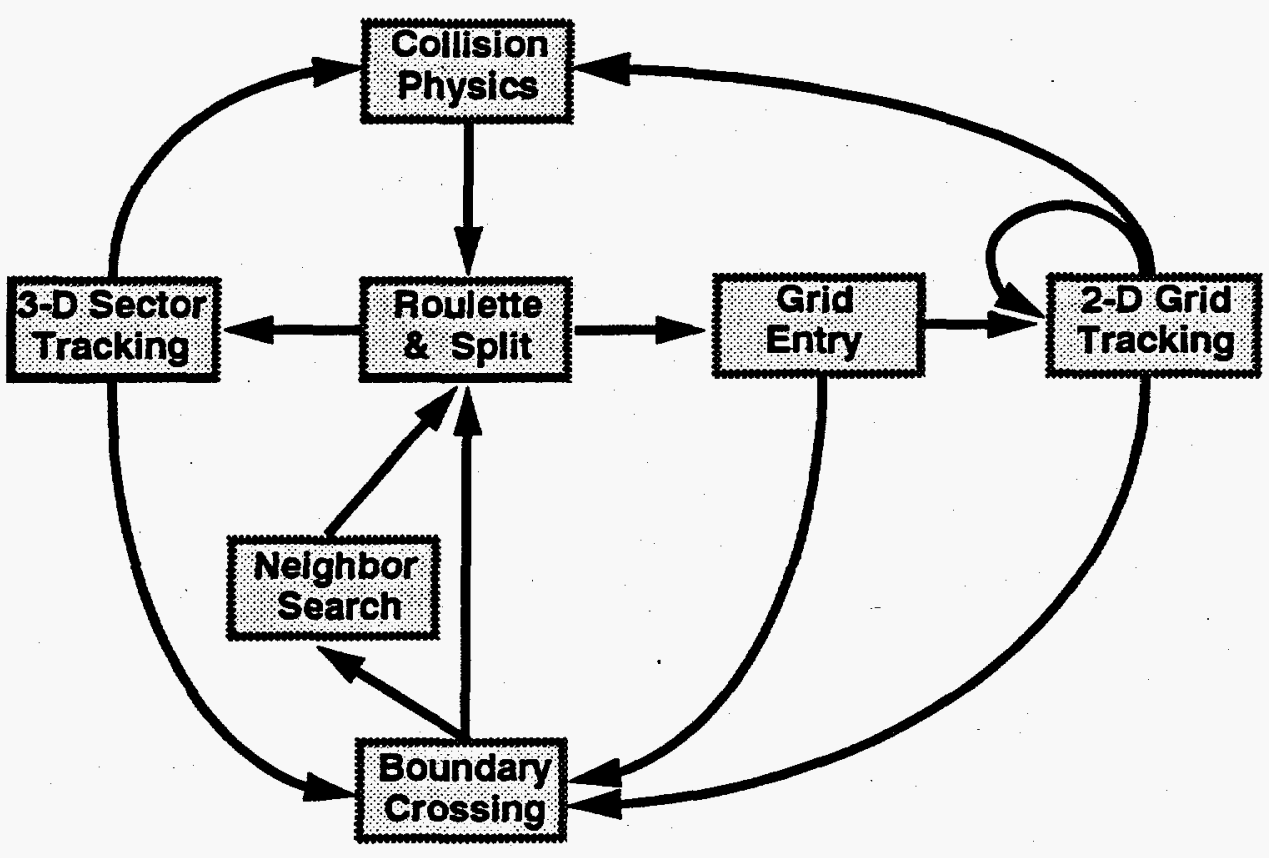

Vector \& Parallel Monte Carlo - Event-driven Algorithm

\section{Monte Carlo - Vectorization}

Event-Driven Algorithm:

\section{Event Queues}

Event 1

Event 2

Event 3

Event 4

otc.

\section{पामा}

पाIIIIII

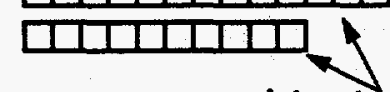

pointers to particles

while ovents are pending:

- - select event $(1,2,3, \ldots)$ with largest event-queue

- - execute the ovent:

- $\quad$ Pull N pointers from event-queue

- $\quad$ Gether attributes for $N$ particles from stack

- - Analyze event - vector calculation

- $\quad$ Scattor moditiod attributos to stack

- Push pointers onto next event-queue

$\bullet$

Particle Stack

Attributes $\rightarrow$ $x, y, z, u, v, w, E, \ldots$

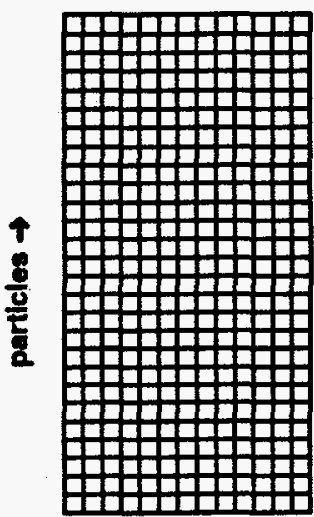




\section{RACER - Code Organization}

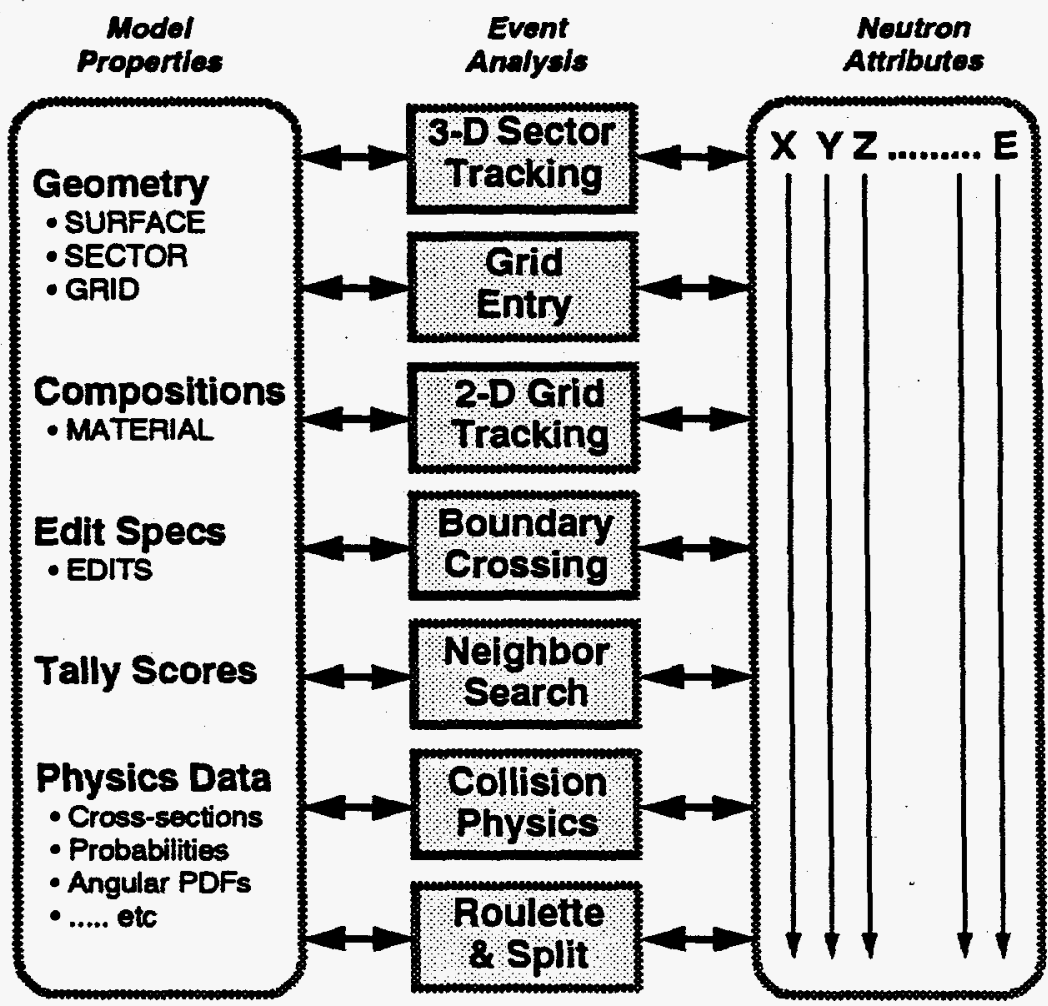

Vector \& Parallal Monte Carlo - Event-driven Algorithm

\section{* Loop over timesteps}

* Loop over batches

- $\quad \rightarrow$ select starters

- * Loop over edit-groups

- $\quad$ - $\rightarrow$ clear edit-group tallies

- - * Loop over super-groups

- $\quad$ - $\rightarrow$ get $\sigma$ 's, $f(\mu) ' s, \ldots .$.

- - . - $\rightarrow$ clear event-queues

- - - - - $\quad \rightarrow \quad$ - $\quad$ - Loop until event-queues are empty

- - - - $\quad \rightarrow$ select event with longest pending queve

- - - - $\quad \rightarrow$ pull from event-queue: pointers to neutrons

- . . . . $\rightarrow$ gather: needed neutron attributes

- - . - $\rightarrow$ analyze event \& tally

-. . . $\rightarrow$ scatter

- $\quad$ - $\quad \rightarrow$ push to next-event queue: pointers to neutrons

- $\bullet \quad \bullet \quad \ldots$

- . . ...

-,$\ldots$

- $\quad \rightarrow$ update eigenvalue, results, stats

- $\cdots$

$\rightarrow$ depletion 


\section{Monte Carlo - Vectorization}

\section{Status}

- Vectorized Monte Carlo, with event-driven algorithms, was proven to work effectively in -1980 on Cray-1 \& Cyber-205

- Large speedups (20x or more) were demonstrated in production Monte Carlo codes, on real problems

- Relatively easy to migrate to MIMD or mixed MIMD/SIMD architectures

- Very few large production codes have adopted this approach

\section{What's the problem ?}

- Must restructure the entire database \& rewrite the entire code

- Large amount of people-time

$\rightarrow$ expensive

- "Why change something that works?" $\rightarrow$ QA work

Vector \& Parallel Monte Cario - Parallel Processing

\section{Parallel Programming}

\section{SIMD Machines}

- Fine-grained parallelism, low-level

- Vector algorithms \& programming

\section{MIMD Machines \& Distributed Systems}

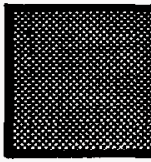

Specify
Physical
Problem
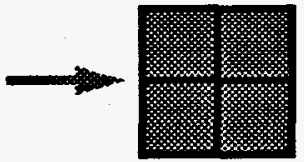

Decompose Computational

Problem
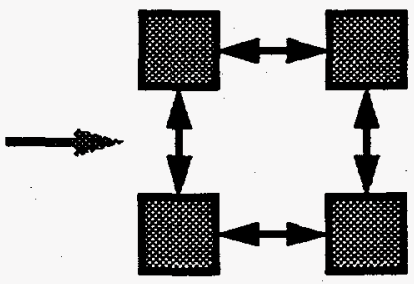

Analyze Sub-domains in Parallel

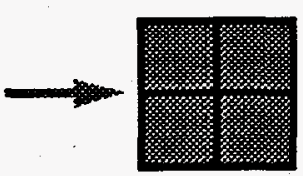

Collect Problem Results

- Coarse-grained parallelism, high-level

- Ideal for loosely-coupled machines \& message-passing libraries pvm, P4, MPI, express, lam, parmacs, ..... 
Physical Problem

- 3-D geometry, continuous-energy physics

- vim-ANL, racer-KAPL, monp-LANL

\section{Conventional Solution Algorithm}

- Random walk for neutrons

- Tally events of interest

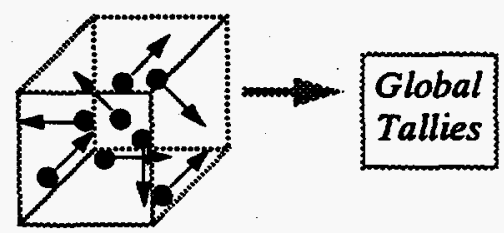

\section{Parallel Algorithm}

- Distribute neutrons to different processors

- Local tallies on each processor

- Combine local tallies into global results

\section{Status}

- racer - in production use,

Cray-C90, Cray-YMP, Meiko-CS1

- vim - in testing,

workstation network \& IBM-SP1

- menp - in production use, workstation network, Cray-YMP

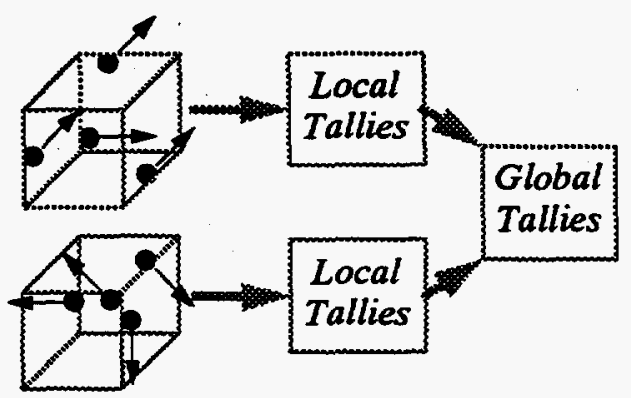

Vector \& Parallel Monte Carlo - Parallel Processing

\section{Monte Carlo - Parallel}

\section{Master-slave approach}

- Master

- Control

- All 1/O

- Slaves

- computations

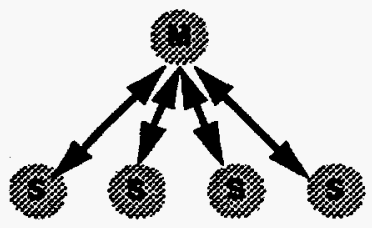

Master process

\begin{tabular}{|c|c|}
\hline $\begin{array}{l}\text { * Loop over timesteps } \\
\text { - } \quad \text { - Loop over batches } \\
\text { - } \quad \rightarrow \text { select starters } \\
\text { - } \quad \text { * Loop over chunks } \\
\text { - } \quad \text { - } \rightarrow \text { find a ready slave } \\
\text { - } \quad \text { - } \quad \text {-. send starters to slave } \\
\text { - } \quad \text { - Loop over slaves } \\
\text { - } \quad \text {-.. } \rightarrow \text { collect slave tallies } \\
\text { - } \quad \rightarrow \text { update eigenvalue, results, stats }\end{array}$ & 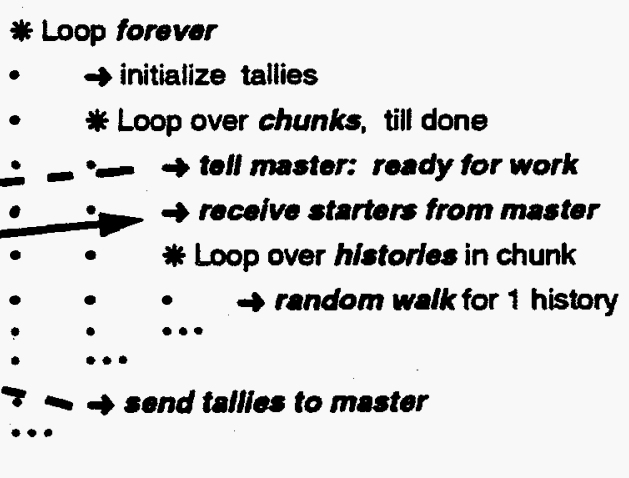 \\
\hline
\end{tabular}




\section{racer - Parallel / Vector Performance}

- Measured performance $\rightarrow$ histories / minute

- 3-D full-core PWR test problem

- Fixed number of histories/processor

- Range in performance spans 1000x [8 hr Cray-C90] [1 yr workstation]

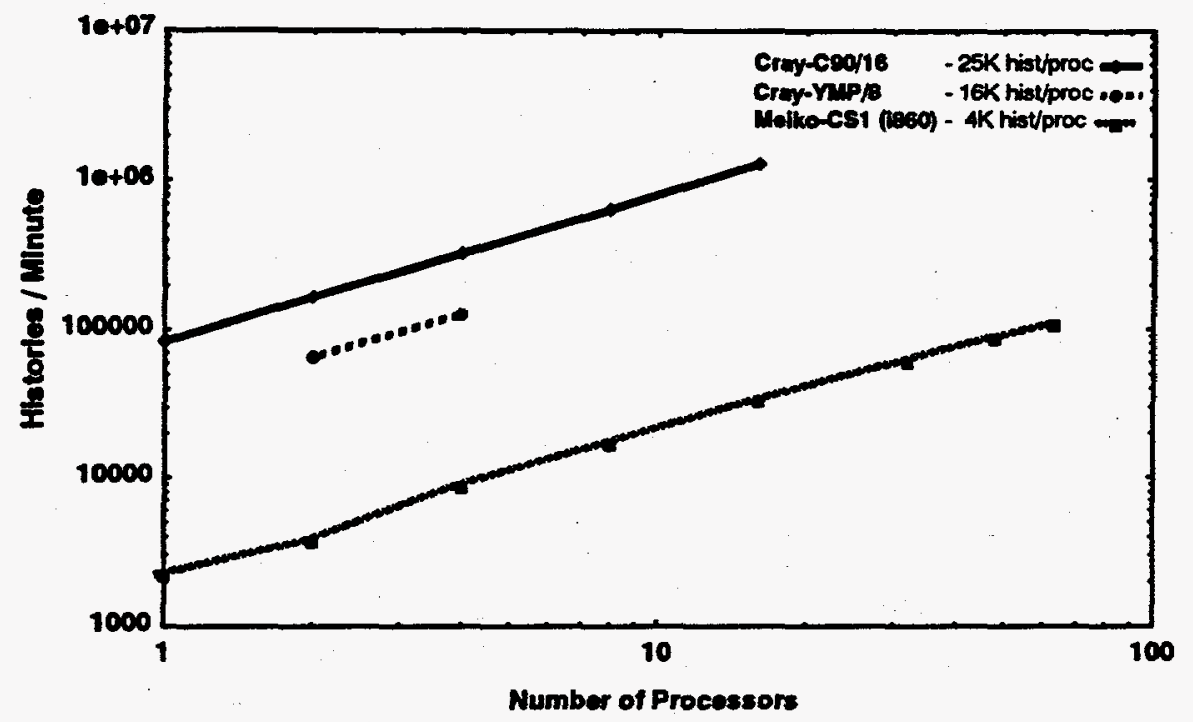

\section{Vector \& Parallel Monte Carlo - Issues}

- Hierarchical parallelism

- Shared memory vs distributed memory

- Parallel speedup \& scaling

- Software \& Portability

- Reproducibility 


\section{Scalar Monte Carlo}

Loop over batches

$\bullet$

- Loop over particles

-. $\bullet$

- - analyze many ovents

- $\quad$ for 1 particle history

$-\infty$

$\infty$

\section{Parallel Scalar Monte Carlo}

Loop over batchas

- Loop over particles, $\mathrm{N}$ at a time

- : scalar scalar ... scalar

- $\quad$ cpu-1 cpu-2 ... cpu-N

-

$\infty$

parallel $\Rightarrow 1$ particle per CPU, scalar analysis

high-tevel: parallel low-level: scalar

\section{Vector Monte Carlo}

Loop over batches

-

- Loop over events

-

- - vector analysis of 1 event

- $\quad$ for many particle historles

-

$\infty$

\section{Parallel Vector Monte Carlo}

Loop over batches

- Loop over events

- : vector vector ... vector

- $\quad$ cpu-1 cpu-2 ... cpu-N

$\infty$

parallel $\Rightarrow$ many particles per CPU vector $\Rightarrow$ events on each CPU

high-level: parallel low-level: vector

Vector \& Parallel Monte Carlo - Hierarchical Parallelism

\section{RACER parallel algorithm}

High-level:

- independent tasks + message-passing

- distribute histories among processors

- Master / Slave algorithm

- Master: control, distribute work, collect results

- Slaves: compute particle histories, no communication with other slaves

\section{Mid-level:}

(next)

- independent tasks + shared memory

- "macro-tasking"

- several slaves share memory, take turns on "critical regions"

\section{Low-level:}

- "microtasking"

- split each DO-100p into pieces, compute, synchronize

\section{Low-level:}

- vectorlzation, within each slave process

- Event-based algorithm (Brown/Martin, 1981)

- vectorize events independently (collision, 3D flight, boundary, ...)

- create \& manage queues of particles waiting for each event 
Shared memory usage

Agorthm decisions:

bsues:

RACER - 1986

- benchmarks

- Cray-XMP

RACER - 1987

- Cray-XMP

RACER - 1988, todaY

- Cray-YMP

- Cray-Cso

- super-Ws

\section{Private data ve. Shared data}

overall memory slze, data cohereney, memory contention, lock/unlock overhead, portability
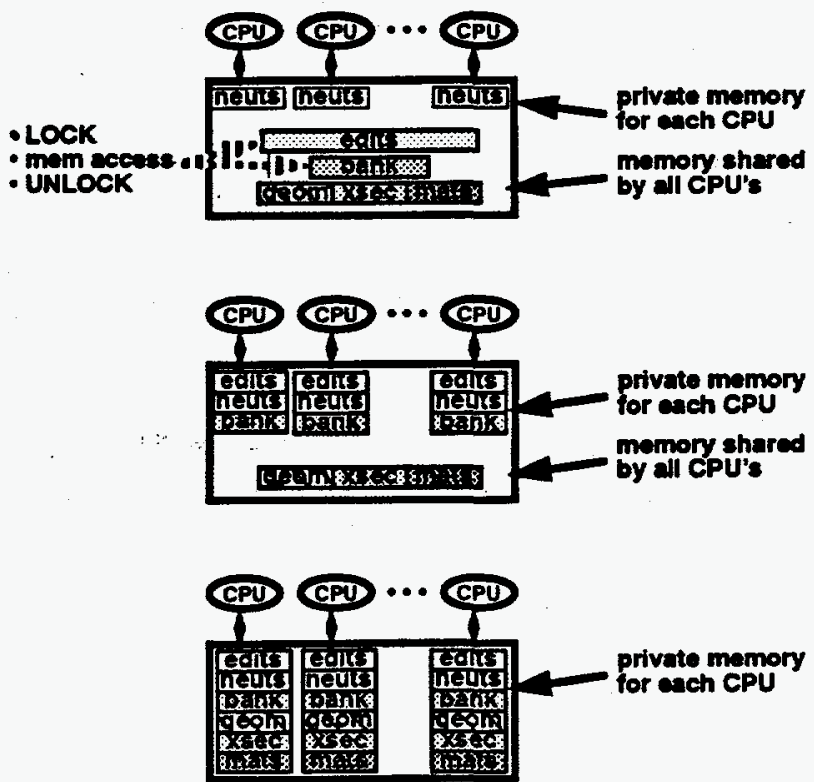

Parallel Monte Carlo Algorithms - Distributed Memory \& Clusters

Distributed memory usage

\author{
Agorithm decisions: \\ bsuos: \\ RACER - 1989 . todaY \\ - Melko CS1, 2 \\ - Ws cluster
}

private data (only)

local memory size

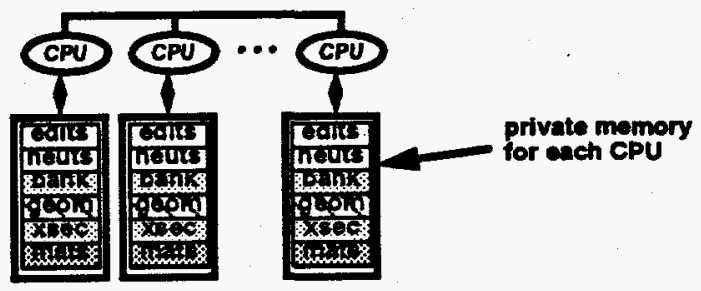

\section{Clustered Shared-Memory}

Agorithm decisions:

bsues:

Private data

vo. Shared data

overall memory size, data coherency, memory contention,

RACER - (soon)

- Next super

- Cray-cso

- cluster of super-Ws

- cluster of anything lock/unlock overhead, portabllity

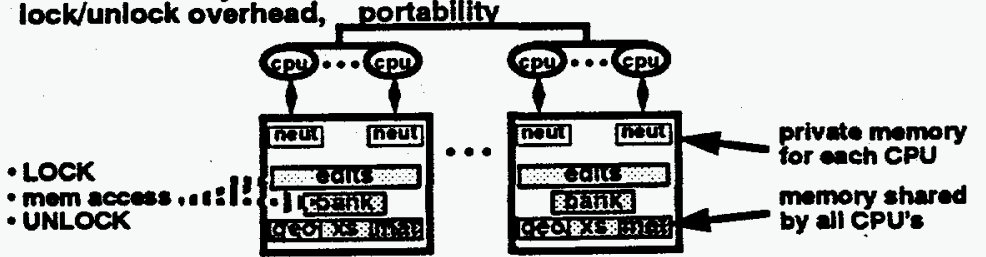




\section{Performance Measurements}

- Metrics

$$
\begin{array}{lll}
\text { Speedup } & S_{N}=T_{1} / T_{N} & N=\# \text { processors } \\
\text { Efficiency } & \eta_{N}=S_{N} / N &
\end{array}
$$

- Fixed Overall Work

- Efficiency decreases with $\mathbf{N}$

- Speedup (eventually) drops

- Example: constant - \# histories/batch

variable - \# histories/processor $(\sim 1 / N)$

- Fixed Work per Processor

- Efficiency approx. constant with $\mathbf{N}$

- Speedup approx. linear with $\mathbf{N}$

- Example: $\quad$ variable - \# histories/batch $(\sim \mathrm{N})$

constant - \# histories/processor

$\rightarrow$ called "scaled speedup"

Master / Slave Algorithm

- Master

- control

- distribute work

- collect results

- Slaves

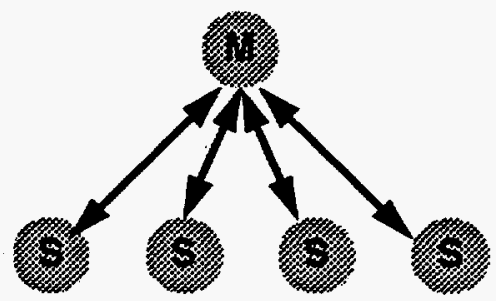

- compute particle histories

- no communication with other slaves

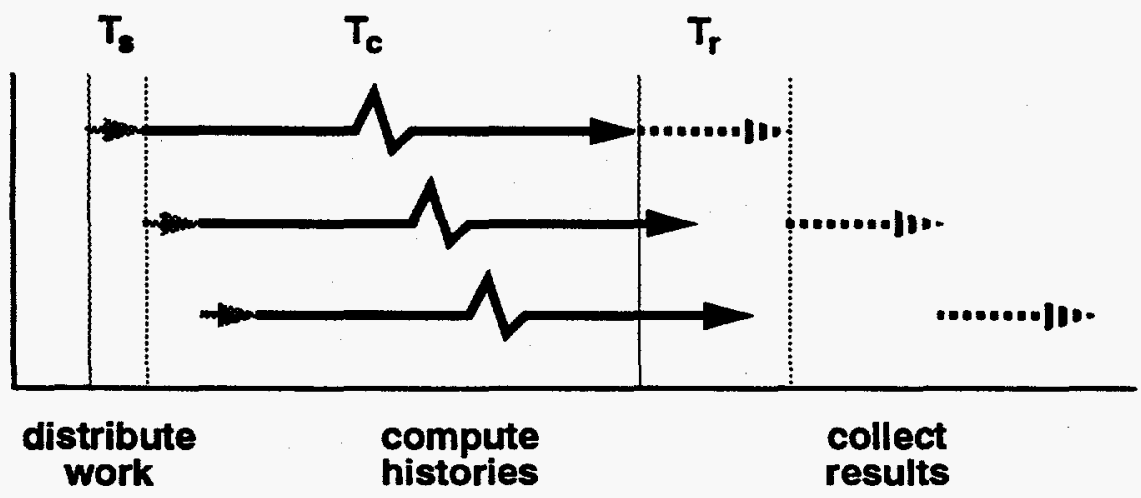


"Parallel" Message Passing

Most parallel computers support concurrent message-passing between separate pairs of nodes
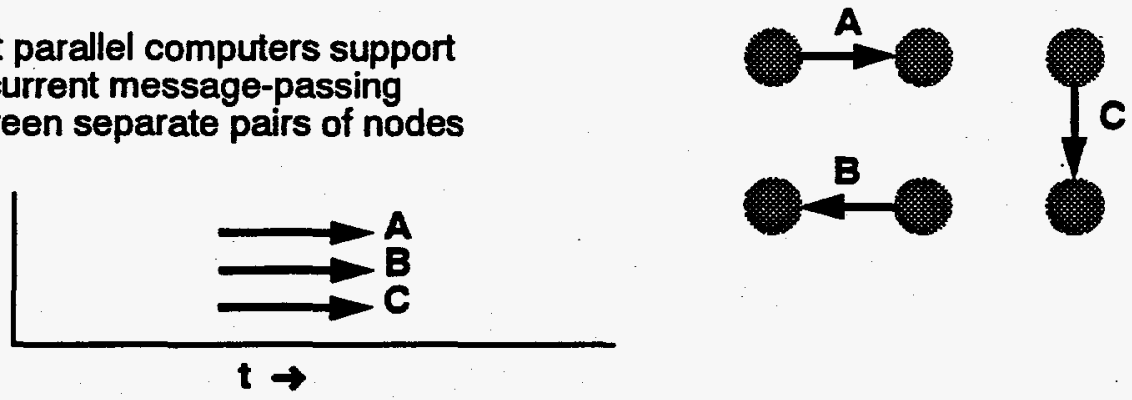

\section{"Serial" Message Passing}

But, multiple messages to a single node are (almost always) handled sequentially
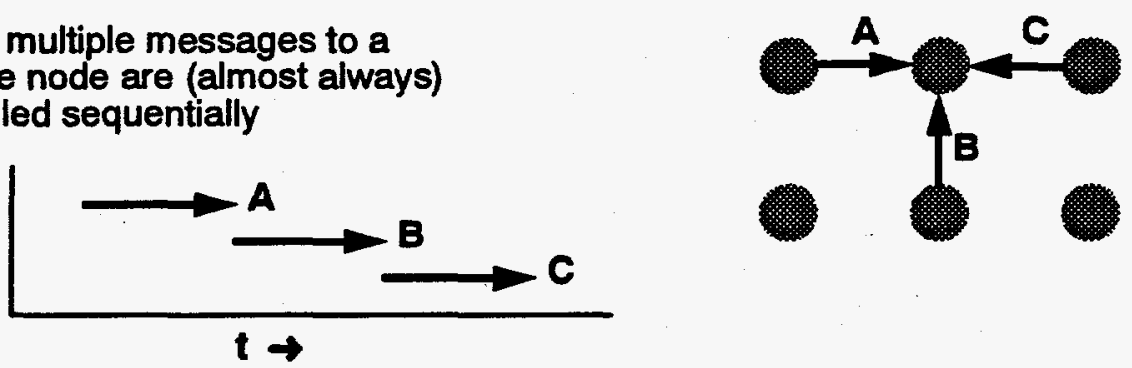

Parallel Speedup \& Scaling

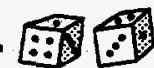

For a given physical problem,

- computation time number of histories $(M)$

- Define:

$$
\begin{aligned}
& M_{1}=\begin{array}{l}
\text { number of histories in job (fix.src.) or batch (eig.) } \\
\text { for calculation using } 1 \text { slave }
\end{array} \\
& N=\text { number of slave processes } \\
& \mathbf{M}_{\mathbf{N}}=\begin{array}{l}
\text { number of histories per slave } \\
\text { in job (fix.src) or batch (eig.) using } N \text { slaves }
\end{array} \\
& T_{N}=\text { total time required for } M_{N} \text { histories }=T_{s}+T_{c}+T_{r}
\end{aligned}
$$

"Fixed Size" Problem: $\quad M_{N}=M_{1} / N$

- Constant number of particles in job (fix.src.) or batch (eig.)

- Goal of parallel calculation:

same work, less time

"Scaled" Problem: $\quad M_{N}=M_{1}$

- Constant number of particles per slave

- Goal of parallel calculation: more work, same time 


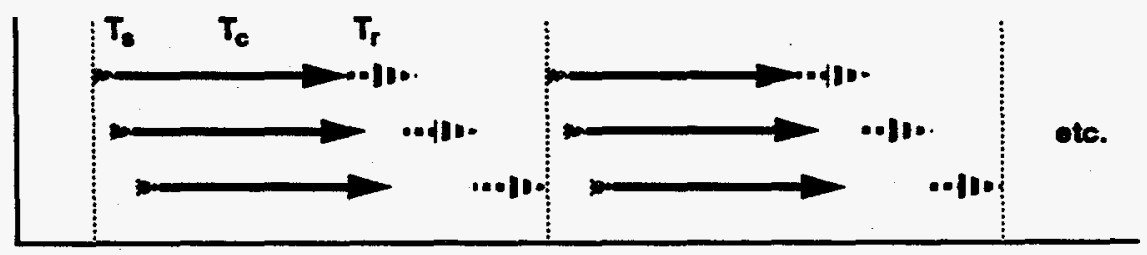

$t_{h}=$ cpu time per history,

depends on: physics, geometry, Fortran compiler, machine speed \& architecture, computer coding, random straggling, etc.

$\mathbf{L}=$ amount of tally data per slave, proportional to \# regions

T. - 0 negligible - send coordinates to slaves

$T_{r}=s+L / r \quad s=$ latency, $r=$ streaming rate

$T_{c} \approx M_{N} t_{h}$

$$
\begin{aligned}
& T_{c}^{f f x}=M_{1} t_{h} / N \quad \text { (fixed size) } \\
& T_{c}^{\text {scale }}=M_{1} t_{h} \quad \text { (scaled) }
\end{aligned}
$$

Scaling Models for Parallel Eigenvalue Calculations

$$
\begin{aligned}
& \text { Eroblem Message } \\
& \text { size Passing } \\
& \text { Rarallel Speedup } \\
& c=(s+L / r) /\left(M_{1} t_{h}\right)
\end{aligned}
$$


Scaling Models for Parallel Eigenvalue Calculations

problem alze

flxed

flxed

scaled

scaled esmmunleations

serial

parallel

serial

parallel
Speedup

$$
S=N /\left(1+\mathrm{CN}^{2}\right)
$$
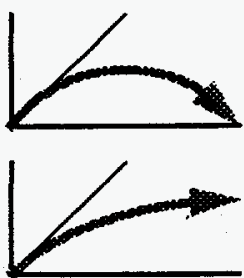

$$
\mathbf{S}=\mathbf{N} /(1+\mathbf{C N})
$$$$
\mathbf{S}=\mathbf{N} /(1+\mathbf{c N})
$$
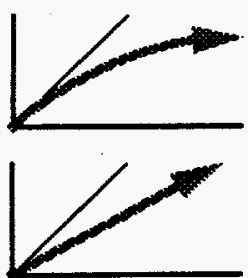

Scaling Models for Parallel Fixed-source Calculations

for long calculations:

$\mathbf{S} \sim \mathbf{N}$

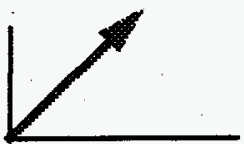

$$
\begin{aligned}
& N=\text { number of slaves } \\
& c=(s+L / r) /\left(M_{1} t_{h}\right)
\end{aligned}
$$

Scaling - Limits 8. Matrics

Parallel Eigenvalue Calculations

Fixed size, serial messages

$$
\mathbf{S}=\mathrm{N} /\left(1+\mathrm{CN}^{2}\right)
$$

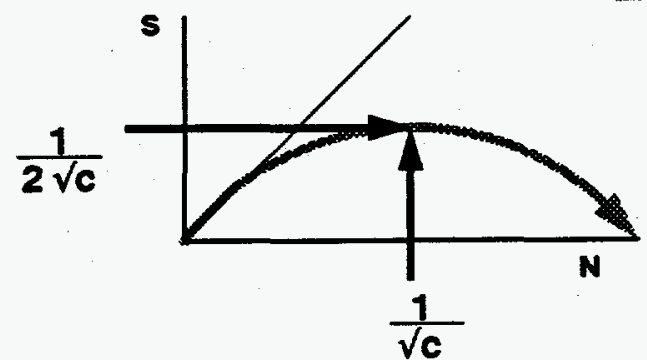

Scaled size, serial messages

$$
S=N /(1+C N)
$$

$1 / 2 c$

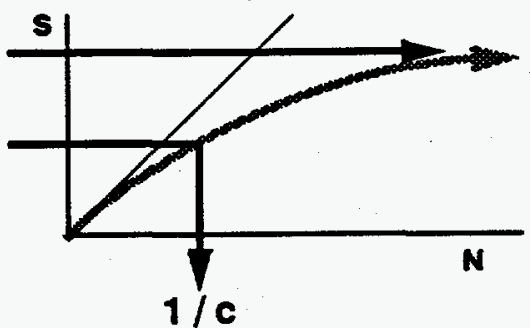

$$
\begin{aligned}
& N=\text { number of slaves } \\
& c=(s+L / r) /\left(M_{1} t_{h}\right)
\end{aligned}
$$


Parallel Elgenvalue Calculations - scaled size, serial messages

$$
\begin{aligned}
& S=N /(1+C N) \\
& S_{\max }=1 / C \\
& N_{1 / 2}=1 / C
\end{aligned}
$$

$$
\begin{aligned}
& N=\text { number of slaves } \\
& c=(s+L / r) /\left(M_{1} t_{h}\right)
\end{aligned}
$$

\section{Examples:}

- VIM, TREAT problem

Sun Sparc2 workstation cluster

$$
\begin{array}{ll}
c=.0043 & S_{\max }=233 \\
c=.011 & S_{\max }=93 \\
c=.014 & S_{\max }=70 \\
c=.00134 & S_{\max }=748
\end{array}
$$
rs6000/350 workstation cluster$$
\text { SP1, using ethernet }
$$

SP1, using EUIH comm.

- RACER, "typical" large problem

$100 \mathrm{~K}$ histories/min, $20 \mathrm{~K}$ histories/slave

$32 \mathrm{MB}$ tally data, $r \sim 1800 \mathrm{MB} / \mathrm{sec}$

Cray-C90, using SSD for messages c c.001 $S_{\max } \sim 1000$ (16 processors, max)

Vector \& Parallel Monte Carlo

\section{Software \& Portability Issues}

Portability

- Best bets (for now) $\rightarrow$ Fortran-77 $+C+$ message-passing

or

$\rightarrow$ Fortran-77 $+\mathrm{C}+++$ message-passing

- Maybe

$\rightarrow$ Fortran-90+C

- Gamble

$\rightarrow$ vendor-specific languages, new languages

- Not likely

$\rightarrow$ "automatic" parallelizers

"Standard" message-passing packages

- pvm

- $m p i$

- p4

- express $\rightarrow$ from Oak Ridge \& Univ. Tennessee

$\rightarrow$ "Message-Passing Interface", draft standard

$\rightarrow$ from Argonne

$\rightarrow$ commercial product, Parasoft

Performance using distributed computing

- Minimize communications

- Minimize disk I/O (master only ???) 


\section{Parallel Monte Carlo - Reproducibility}

\section{Eigenvalue Calculations}

- Power iteration

$\rightarrow$ Histories within a batch are independent - analyze in parallel

$\rightarrow$ Successive batches are not independent - analyze sequentially

- Large batch size

$\rightarrow$ maximize parallel efficiency \& performance

$\rightarrow$ Side effect: reduced bias in M.C. eigenvalue \& shapes

\section{Reproducibility}

- Must get identical results (bit-for-bit) using 1 or many processors

- A solved problem, see

"Reproducibility \& Monte Carlo Eigenvalue Problems,"

F. B. Brown \& T. M. Sutton, Trans. Am. Nucl. Soc. 65, 235 (June 1992)

$\rightarrow$ special random number generators

$\rightarrow$ reorder fission-site bank at end of batch

Reproducibility \& Monte Carlo Eigenvalue Calculations

- Difficulties: - Random number usage by each particle - Ordering of particles in "fission bank"
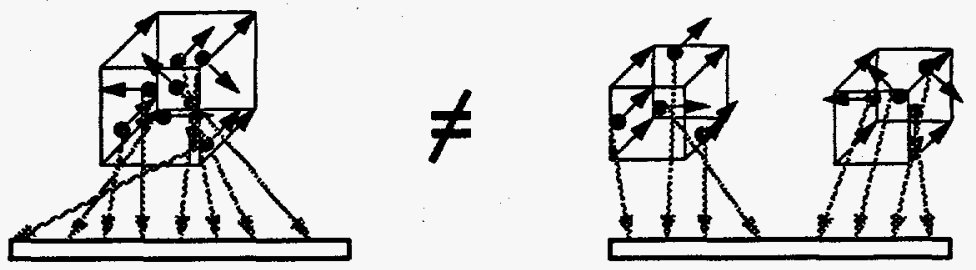

- Require identical results if calculation repeated

(with same random numbers)

- with different number of processors

- with different vector length limits

- with different "supergrouping" of cross-section data

$\rightarrow$ use a separate random seed for each particle

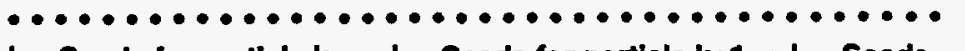

$\mid \leftarrow$ Seeds for particle $k \rightarrow \mid \leftarrow$ Seeds for particle $k+1 \rightarrow \mid \leftarrow$ Seeds .....

$\rightarrow$ unique reordering of fission bank at end of each batch

Brown \& Sutton, Trans. Am. Nucl. Soc. 65, 235 (1992) 
RACER Monte Carlo $\Rightarrow 3-D$ reactor physics analysis

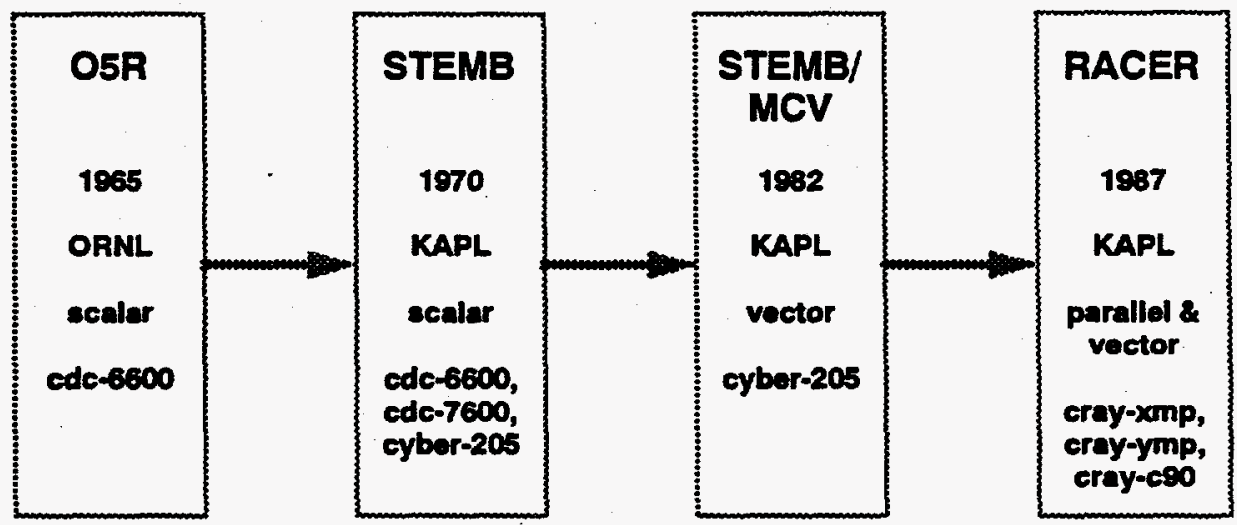

- Extensively benchmarked vs experiment \& real reactors

- Most-used KAPL code: $\quad-30-40 \%$ of all Cray time

\section{Focus for RACER development:}

$\Rightarrow$ very large \& detailed 3-D reactor physics problems

RACER Monte Carlo System - Modules

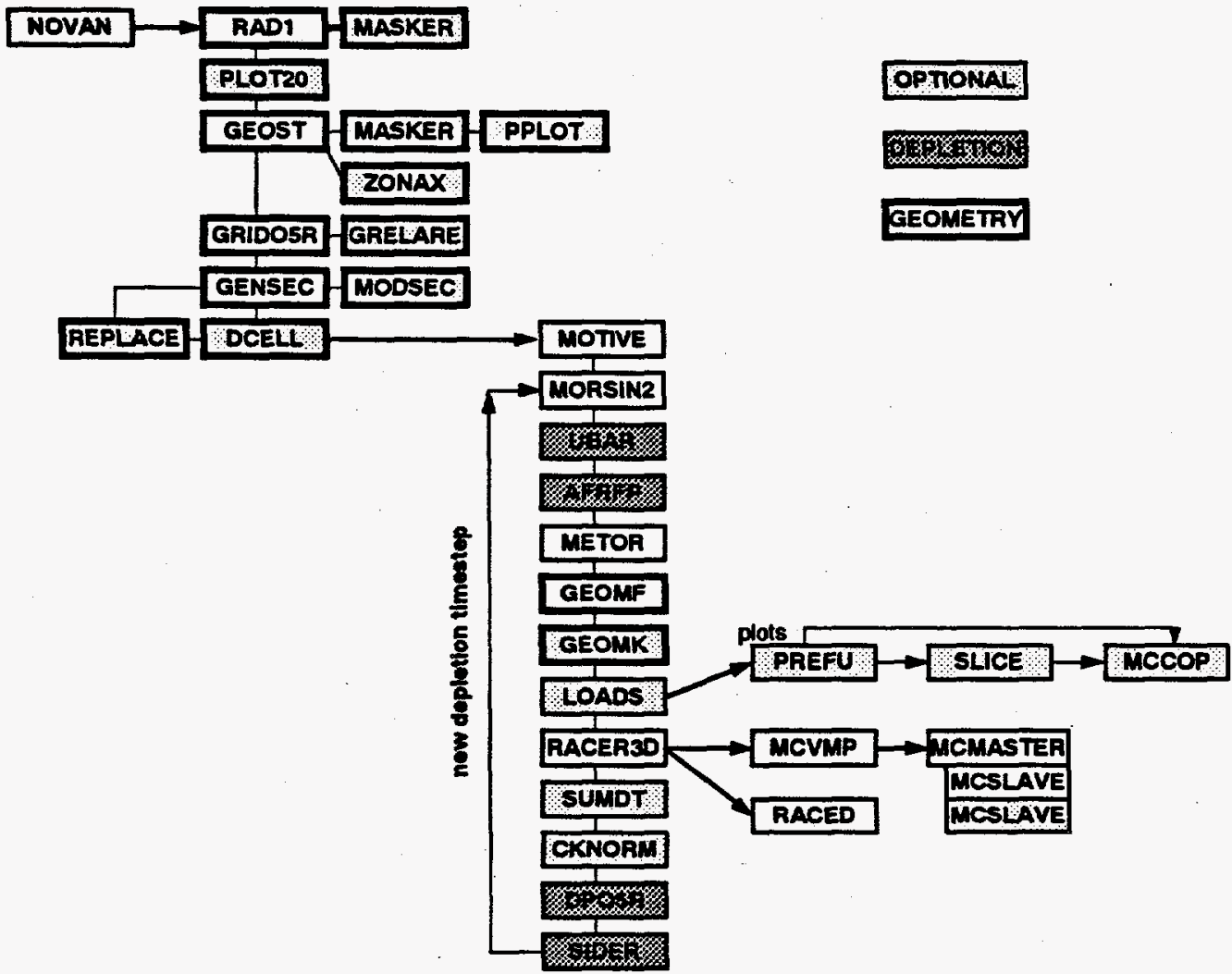


Module Development History ～(dates are very approximate)

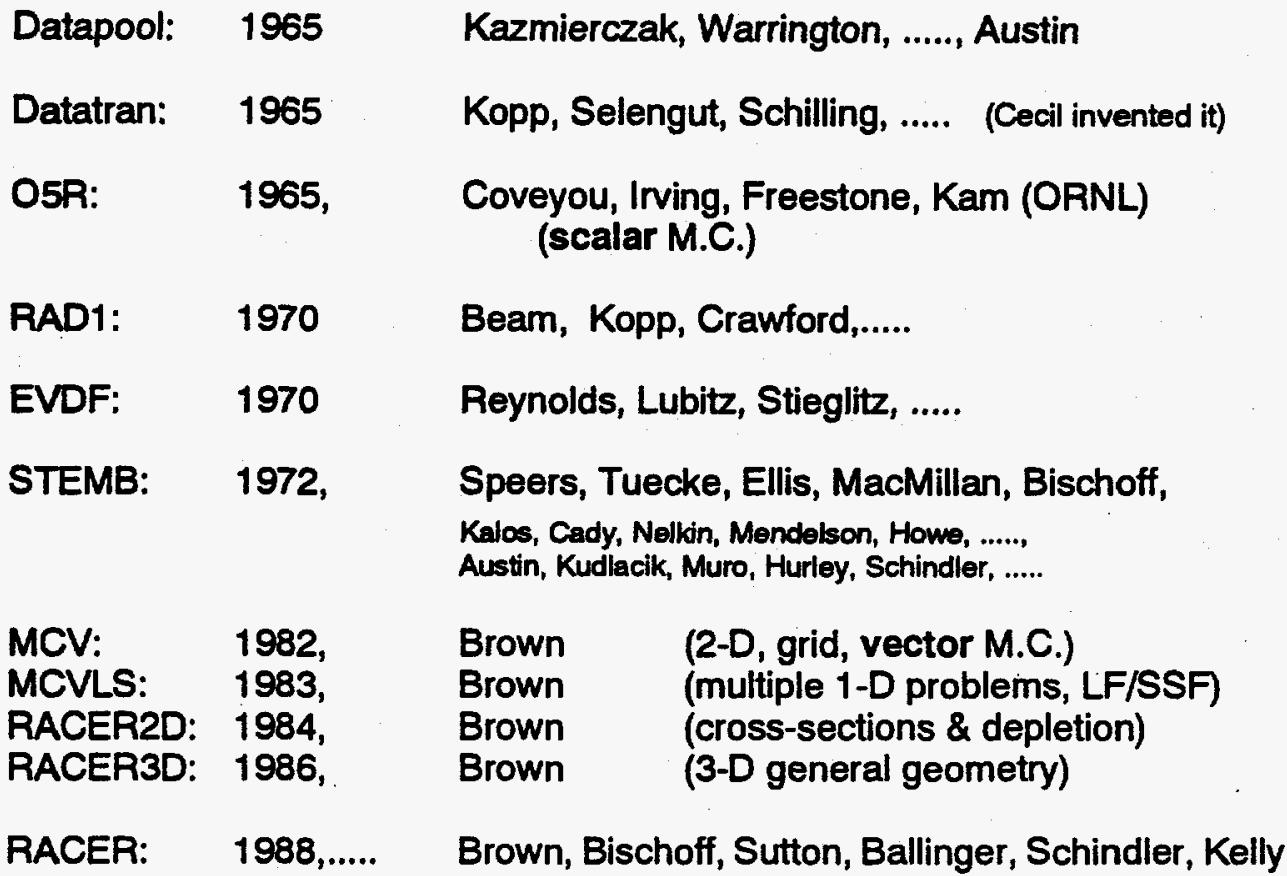

RACER Monte Carlo System - Modules

$\begin{array}{ll}\text { NOVAN } & \text { - initialize Datapool \& read thermal multigroup cross-sections } \\ \text { RAD1 } & -2-D \text { geometry setup } \\ \text { PLOT20 } & -2-D \text { pictures } \\ \text { GEOST } & - \text { construct 3-D geometry from 2-D RAD1 model (MASKER, PPLOT, ZONAX) } \\ \text { GRIDO5R } & - \text { process 2-D RAD1 geometry into "grids" (GRELARE) } \\ \text { GENSEC } & -3-D \text { surface equations \& sector definitions (MODSEC, REPLACE) } \\ \text { DCELL } & - \text { subdivide 2-D M.C. geometry \& edits to match NOVA } \\ \text { MOTNE } & - \text { prepare description of cross-section data, or TAPE9/TAPE3 libraries } \\ \text { MORSIN2 } & - \text { assign edit media, compositions, importances, source, ..... } \\ \text { UBAR } & - \text { linkage from NOVA depletion to Monte Carlo } \\ \text { AFRFP } & - \text { setup lumped multigroup background fission product cross-sections } \\ \text { METOR } & - \text { setup energy mesh, edits (isotopic, comb, ratio, .....), edit groups, \& misc. input } \\ \text { GEOMF } & - \text { reformat 3-D geometry data } \\ \text { GEOMK } & - \text { compute areas \& volumes }\end{array}$




$\begin{array}{ll}\text { LOADS } & \text { - edit material loadings } \\ \text { PREFU } & \text { - collect data for plotting } \\ \text { SLICE } & \text { - 2-D slice plots, line drawings } \\ \text { MCCOP } & -2-0 \text { slice plots, color } \\ \text { RACER3D } & \text { - collect \& reformat data for Monte Carlo } \\ \text { MCVMP } & \text { - start the Monte Carlo programs } \\ \text { MCMASTER } & - \text { master process for Monte Carlo } \\ \text { MCSLAVE } & \text { - slave process for Monte Carto } \\ \text { RACED } & \text { - edit the results from Monte Carlo } \\ \text { CNVRE } & \text { - convergence plots, selected data vs batch } \\ \text { SUMDT } & \text { - retrieve selected results into Datatran lists } \\ \text { CKNORM } & \text { - detailed comparison of Monte Carlo \& diffusion results } \\ \text { DPOSR } & \text { - linkage from Monte Carlo to NOVA depletion } \\ \text { SIDER } & \text { - depletion }\end{array}$

- Parallel Monte Carlo codes ( mcnp4a, keno, vim, racer, ... ) are now running on many parallel computers (cray, meiko, intel, convex, cm5, ...) \& workstation networks

- Master/slave algorithms are simple, easy to implement, \& scale well for eigenvalue problems.

- Communications bottlenecks at the master process are not a problem today:

- ethernet is fast enough for 10's of slaves

- FDDI, EUIH, \& other schemes should permit 100's of slaves

- The major limitation on parallel Monte Carlo today appears to be memory size - each node must contain entire problem \& tallies 
Vector \& parallel computing have side-benefits:

- reduces convergence problems

- Larger batches + more batches $\rightarrow$ - reduces bias

- better correlation corrections

- "Unthinkable" problems become routine

\section{Proven Monte Carlo algorithms exist for}

- SIMD, MIMD, \& mixed MIMD/SIMD supercomputers \& MP computers

- MIMD distributed processing on a network of machines

Parallel algorithms which scale to 1,000 's or $1,000,000$ 's of nodes

- "master-siave" algorithm on 1,000,000 Intemet nodes could take - 1 year to start

- UNIX socket connections - limits master to 10's or 100's of nodes

$\rightarrow$ develop hierarchical parallelism, "clusters-of-clusters"

$\rightarrow$ parallel histories + geometric decomposition (?)

Algorithms with load-balancing \& fault-tolerance

- "Virtual supercomputer" can change continuously

$\rightarrow$ recover from lost nodes \& hardware failures

$\rightarrow$ dynamic load balancing

$\rightarrow$ cooperate with distributed queuing systems

Acceleration for eigenvalue calculations

$\rightarrow$ automated procedures for discarding initial batches

$\rightarrow$ importance sampling or "fission matrix"

Methods for eliminating bias

Improved methods for estimating variance, corrections for correlation 


\section{References}

\section{General References on Monte Carlo Methods for Particle Transport Problems}

[1] L. L. Carter and E. D. Cashwell, Particle Transport Simulation with the Monte Carlo Method, ERDA Critical Review Series, TID-26607, National Technical Information Service, Springfield MA (1975).

[2] E. D. Cashwell and C. J. Everett, A Practical Manual on the Monte Carlo Method for Random Walk Problems, Pergamon Press, London (1959).

[3] J. J. Duderstadt and W. R. Martin, Transport Theory, John Wiley \& Sons, NY (1979).

[4] G. Goertzel and M. H. Kalos, "Monte Carlo Methods in Transport Problems," in Progress in Nuclear Energy, Series I, Physics and Mathematics, Vol. 2, (1958).

[5] J. H. Halton, "A Retrospective and Prospective Survey of the Monte Carlo Method," SIAM Rev. 12, 1, (1970).

[6] J. M. Hammersley and D. C. Handscomb, Monte Carlo Methods, John Wiley \& Sons, NY (1964).

[7] M. H. Kalos and P. A. Whitlock, Monte Carlo Methods, Volume I: Basics, John Wiley \& Sons, NY (1986).

[8] E. E. Lewis and W. F. Miller, Jr., Computational Methods of Neutron Transport, American Nuclear Society, Inc., LaGrange Park, IL (1993).

[9] I. Lux and L. Koblinger, Monte Carlo Particle Transport Methods: Neutron and Photon Calculations, CRC Press, Ann Arbor, MI (1991).

[10] S. Nakamura, Computational Methods in Engineering and Science, R. E. Krieger Pub. Company, Malabar, FL (1986).

[11] R. Y. Rubinstein, Simulation and the Monte Carlo Method, John Wiley \& Sons, NY (1981).

[12] Y. A. Schreider, The Monte Carlo Method, Pergamon Press, NY (1966).

[13] J. Spanier and E. M. Gelbard, Monte Carlo Principles and Neutron Transport Problems, Addison-Wesley, Reading, MA (1969).

[14] J. Wood, Computational Methods in Reactor Shielding Pergamon Press, Oxford (1982).

General References on Random Number Generation and Random Sampling Methods

[15] L. Devroye, Non-Uniform Random Variate Generation, Springer-Verlag, NY (1986).

[16] C. J. Everett and E. D. Cashwell, "A Third Monte Carlo Sampler," LA9721-MS, Los Alamos National Laboratory, Los Alamos, NM (1983).

[17] H. Kahn, "Applications of Monte Carlo," AECU-3259, Rand Corporation, Santa Monica, CA (1954).

[18] D. E. Knuth, The Art of Computer Programming, Vol. 2: Semi-numerical Algorithms, Addison-Wesley, Reading, MA (1981).

[19] E. J. McGrath and D. C. Irving, "Techniques for Efficient Monte Carlo Simulation," ORNL-RSIC-38, Vols. I-III, Oak Ridge National Laboratory, Oak Ridge, TN (1975). 
General References on Monte Carlo Methods for the Solution of Reactor Eigenvalue Problems

[20] R. C. Gast and N. R. Candelore, "Monte Carlo Eigenfunction Strategies and Uncertainties," in Proc. NEACRP Meeting of a Monte Carlo Study Group, ANL-75-2, Argonne National Laboratory, Argonne, IL (1974).

[21] D. C. Irving, R. M. Freestone, and F. B. K. Kam, "O5R, A General Purpose Neutron Monte Carlo Code," ORNL-3622, Oak Ridge National Laboratory (1965).

[22] M. H. Kalos, F. R. Nakache, and J. Celnik, "Monte Carlo Methods in Reactor Computations," in Computing Methods in Reactor Physics, Gordon and Breach, NY (1968).

[23] J. Lieberoth, "A Monte Carlo Technique to Solve the Static Eigenvalue Problem of the Boltzmann Transport Equation," Nukleonik 11, 213 (1968).

[24] M. R. Mendelson, "Monte Carlo Criticality Calculations for Thermal Reactors," Nucl. Sci Eng. 32, 319-331 (1968).

[25] H. Rief and H. Kschwendt, "Reactor Analysis by Monte Carlo," Nucl. Sci. Eng., 30, 395 (1967).

[26] E. R. Woodcock, et al, "Techniques Used in the GEM Code for Monte Carlo Neutronics Calculations in Reactors and Other Systems of Complex Geometry," in Proc. Conf. Applications of Computing Methods to Reactor Problems, ANL-7050, Argonne National Laboratory, Argonne, IL (1965).

Open-Literature Publications on the RACER Monte Carlo Code, Vector and Parallel Monte Carlo, and Related Monte Carlo Methods

[27] D. J. Kelly, "Depletion of a BWR Lattice Using the RACER Continuous-Energy Monte Carlo Code," Proceedings of the ANS Intl. Conf. on Mathematics \& Computation, Reactor Physics, \& Environ. Analyses, April 30-May 4, Portland, Oregon (1995).

[28] C. T. Ballinger, "The Direct $S(\alpha, \beta)$ Method for Thermal Neutron Scattering," Proceedings of the ANS Intl. Conf. on Mathematics \& Computation, Reactor Physics, \& Environ. Analyses, April 30-May 4, Portland, Oregon (1995).

[29] F. B. Brown, "Random Number Generation with Arbitrary Strides," Trans. Am. Nucl. Soc. 71, 202 (1994).

[30] S. Matsuura, F. B. Brown, and R. N. Blomquist, "Parallel Monte Carlo Eigenvalue Calculations," Trans. Am. Nucl. Soc. 71, 199 (1994).

[31] T. M. Sutton and F. B. Brown, "Parallel Monte Carlo for Reactor Calculations," Proceedings of the ANS Topical Meeting on Advances in Reactor Physics, April 11-15, 1994, Knoxville, TN (April 1994).

[32] F. B. Brown, K. L. Derstine, and R. N. Blomquist, "Distributed Computing and Nuclear Reactor Analysis," Proceedings of the ANS Topical Meeting on Advances in Reactor Physics, April 11-15, 1994, Knoxville, TN (April 1994).

[33] R. N. Blomquist and F.B. Brown, "Parallel Monte Carlo Reactor Neutronics," Proceedings of the Society for Computer Simulation Meeting on High Performance Computing '94, April 11-15, 1994, La Jolla, CA (April 1994).

[34] F. B. Brown and J. L. Vujic, "Comparison of Direct and Rejection Sampling Methods," Trans. Am. Nucl. Soc. 69, 223 (Nov. 1993). 
[35] E. M. Gelbard, F. B. Brown, and A. G. Gu, "Estimation of Fission Source Bias in Monte Carlo Eigenvalue Calculations," Trans. Am. Nucl. Soc. 69, 201 (Nov. 1993).

[36] F. B. Brown, "Monte Carlo Neutronics Simulations on Parallel and Distributed Computers," invited paper presented at the SIAM Conference on Simulation and Monte Carlo Methods, San Francisco (Aug. 1993).

[37] F. B. Brown and T. M. Sutton, "Reproducibility and Monte Carlo Eigenvalue Calculations," Trans. Am. Nucl. Soc. 65, 235 (June 1992).

[38] R. G. Gamino, F. B. Brown, and M. R. Mendelson, “A Monte Carlo Green's Function Method for 3-D Neutron Transport," Trans. Am. Nucl. Soc 65, 237 (June 1992).

[39] W. R. Martin, J. A. Rathkopf, and F. B. Brown, "The Impact of Advances in Computer Technology on Particle Transport Monte Carlo," Proceedings of the ANS Topical Meeting on New Horizons in Radiation.Protection and Shielding, Richland WA (April 1992).

[40] F. B. Brown, W. R. Martin, and J. A. Rathkopf, "Particle Transport Monte Carlo and Parallel Computers," Proceedings of the Argonne Theory Institute on Parallel Monte Carlo Simulations, Argonne National Laboratory (August 1991).

[41] W. R. Martin and F. B. Brown, "Monte Carlo Methods for Particle Transport," Trans. Am. Nucl. Soc. 60, 336 (1989).

[42] F. B. Brown and F. G. Bischoff, "Computational Geometry for Reactor Applications," Trans. Am. Nucl. Soc. 57, 112 (1988).

[43] F. B. Brown, "Present Status of Vectorized Monte Carlo," Trans. Am. Nucl. Soc. 55, 323 (1987).

[44] W. R. Martin and F. B. Brown, "Present Status of Vectorized Monte Carlo for Particle Transport Analysis," International Journal of Supercomputer Applications, Vol. 1, No. 2, 11-32 (June 1987).

[45] F. B. Brown, "Vectorization of 3-D General-Geometry Monte Carlo," Trans. Am. Nucl. Soc. 53, 283 (1986).

[46] F. B. Brown and W. R. Martin, "Monte Carlo Methods for Vector Computers," J. Progress in Nuclear Energy, Vol. 14, No. 3, 269-299 (1984).

[47] F. B. Brown and M. R. Mendelson, "Vectorized Monte Carlo Applications in Reactor Physics Analysis," Trans. Am. Nucl. Soc. 46, 727 (June 1984).

[48] F. B. Brown, "Vectorized Monte Carlo Methods for Reactor Lattice Analysis," Proceedings ANS Topical Meeting on Advances in Reactor Computations, Salt Lake City, Utah, 108-123 (March 1983).

[49] F. B. Brown, "Vectorized Monte Carlo Methods for Reactor Lattice Analysis," KAPL4163, Knolls Atomic Power Laboratory, Schenectady, NY (March 1983).

[50] F. B. Brown, "Development of Vectorized Monte Carlo Algorithms for Reactor Lattice Analysis," Trans. Am. Nucl. Soc. 43, 377 (1982).

[51] F. B. Brown, "Vectorized Monte Carlo," Ph. D. dissertation, University of Michigan, Ann Arbor, Michigan (1981).

[52] F. B. Brown, W. R. Martin, and D. A. Calahan, "Investigation of Vectorized Monte Carlo Algorithms," Trans. Am. Nucl. Soc. 39, 755 (1981).

[53] F. B. Brown, W. R. Martin, and D. A. Calahan, “A Discrete Sampling Method for Vectorized Monte Carlo Algorithms," Trans. Am. Nucl. Soc. 38, 354 (1981).

[54] F. B. Brown, D. A. Calahan, W. R. Martin, et al, "Investigation of Vectorized Monte Carlo Algorithms" working paper presented at the DOE Conference on High Speed Computing, Gleneden Beach, Oregon (April 1981). 
[55] C. L. Ellis and D. B. MacMillan, "O5R Users Manual," KAPL-M-6741, National Technical Information Service, 5285 Port Royal Road, Springfield, VA 22161 (1967). 


\section{DISCLAMMER}

This report was prepared as an account of work sponsored by an agency of the United States Government. Neither the United States. Government nor any agency thereof, nor any of their employees, makes any warranty, express or implied, or assumes any legal liability or responsibility for the accuracy, completeness, or usefulness of any information, apparatus, product, or process disclosed, or represents that its use would not infringe privately owned rights. Reference herein to any specific commercial product, process, or service by trade name, trademark, manufacturer, or otherwise does not necessarily constitute or imply its endorsement, recommendation, or favoring by the United States Government or any agency thereof. The views and opinions of authors expressed herein do not necessarily state or reflect those of the United States Government or any agency thereof. 